MANIPULATING SURFACE NANOBUBBLES

SHANGJIONG YANG 
Composition of The Graduation Committee

Chairman

Prof. dr. L. van Wijngaarden

Promoters

Prof. dr. rer. nat. D. Lohse

Prof. dr. H. J. W. Zandvliet

Members

Prof. dr. A. A. Darhuber

Dr. E. S. Kooij

Prof. dr. B. Poelsema

Prof. dr. H. Schönherr

Prof. dr. O. I. Vinogradova

\section{FOM}

This work is part of the research program of the Stichting voor Fundamenteel Onderzoek der Materie (FOM), which is financially supported by the Nederlandse Organisatie voor Wetenschappelijk Onderzoek (NWO). It was carried out at the Physics of Fluids group and Solid State Physics group in Faculty of Science and Technology, University of Twente.

Publisher: Shangjiong Yang, Physics of Fluids, University of Twente, P. O. Box 217, 7500AE Enschede, The Netherlands. http://pof.tnw.utwente.nl

Cover design: Shangjiong Yang. 3D AFM image of surface nanobubbles on silanated wafer under aqueous sodium chloride solution.

Print: Gildeprint B. V., Enschede, The Netherlands.

CC Copyright 2008 by Shangjiong Yang. All rights reserved. No part of this publication may be reproduced, stored in a data base or retrieval system or distributed in any form or by any means, without the prior written permission of the publisher.

ISBN 978-90-365-2728-6 


\title{
MANIPULATING SURFACE NANOBUBBLES
}

\section{DISSERTATION}

\author{
to obtain \\ the degree of doctor at the University of Twente, \\ on the authority of the rector magnificus, \\ prof. dr. W. H. M. Zijm, \\ on account of decision of the graduation committee, \\ to be publicly defended \\ on Thursday, 09 October 2008 at 15:00
}

by

Shangjiong Yang

born on 03 March 1980

in Chongqing, China 
iv

This dissertation has been approved by the promoters:

Prof. Dr. Detlef Lohse

Prof. Dr. Harold J. W. Zandvliet 
to my parents and teachers... 


\section{Contents}

1 Introduction 1

1.1 General Introduction $\ldots \ldots \ldots \ldots$

1.2 Nanobubbles Are Puzzling . . . . . . . . . . . . . . . 4

1.3 Guide through The Chapters . . . . . . . . . . . . 7

References ................... 11

2 Experimental Instrument: Atomic Force Microscope 13

2.1 Introduction $\ldots \ldots \ldots \ldots \ldots \ldots$

2.2 Working Principle of AFM $\ldots \ldots \ldots \ldots$

2.3 The Standard Operation Modes . . . . . . . . . . . . . 16

2.3.1 Contact mode . . . . . . . . . . . . . . . 16

2.3.2 Non-contact mode . . . . . . . . . . . . . . 19

2.3.3 Tapping mode . . . . . . . . . . . . . . . 20

2.4 What Beyond Topographic Imaging . . . . . . . . . . . . . 21

2.4 .1 Phase imaging . . . . . . . . . . . . . 21

2.4 .2 Force curves . . . . . . . . . . . . . . . 22

2.5 Comparisons with Other Microscopes . . . . . . . . . 23

2.6 Tapping AFM in Liquids $\ldots \ldots \ldots$

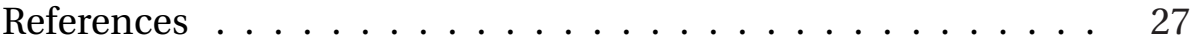


3 Characterization of Nanobubbles on Hydrophobic Surfaces in Water 29

3.1 Introduction . . . . . . . . . . . . . 30

3.2 Experimental Section $\ldots \ldots \ldots \ldots$

3.2.1 Substrate preparation $\ldots \ldots \ldots \ldots$

3.2 .2 AFM imaging . . . . . . . . . . . . 33

3.3 Results and Discussions $\ldots \ldots \ldots \ldots$

3.3.1 Nanobubbles next to a microbubble . . . . . . . 33

3.3.2 Nanobubble stability . . . . . . . . . . . . . 35

3.3.3 Nanobubble 'sliding' along a groove . . . . . . . . . 35

3.3.4 In situ creation of nanobubbles by substrate heating 38

3.3.5 Increase of water temperature . . . . . . . . . . 41

3.3.6 Pressurizing the water with $\mathrm{CO}_{2} \ldots \ldots \ldots \ldots 4$

3.3.7 Influence of cleaning procedure . . . . . . . 46

3.3.8 Adding a surfactant . . . . . . . . . . . . . 48

3.3.9 Ethanol-water exchange . . . . . . . . . . 48

3.3.10 Nanobubbles in $\mathrm{NaCl}$ solution . . . . . . . . . . . 51

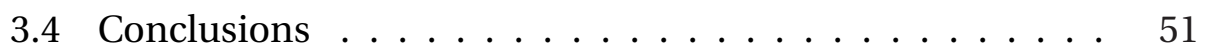

References ........................ 53

4 Correlation between Geometry and Nanobubble Distribution on HOPG Surfaces $\quad 55$

4.1 Introduction . . . . . . . . . . . . . . 56

4.2 Experimental Section . . . . . . . . . . . 57

4.3 Results and Discussions $\ldots \ldots \ldots \ldots \ldots$

4.3.1 Hydrophobicity varies at atomic steps of HOPG surface ................... 57

4.3.2 Ethanol-water-exchange process sufficiently produces nanobubbles on HOPG . . . . . . . . . . . . . 58 
4.3.3 Correlation between surface topography and nanobubble formation . . . . . . . . . . . 60

4.3.4 Deformability of nanobubbles . . . . . . . . . 63

4.4 Conclusions . . . . . . . . . . . . . . 67

References ......................... 68

5 Electrolytically Generated Nanobubbles on HOPG Surfaces 69

5.1 Introduction . . . . . . . . . . . . . 70

5.2 Experimental Section . . . . . . . . . . . . . . 72

5.3 Results and Discussions _. . . . . . . . . . . . . . 74

5.3.1 Nanobubbles by electrolysis of water: dependence on applied voltage and gas type . . . . . . . . . . 74

5.3.2 Nanobubbles in dynamic equilibrium . . . . . . 79

5.3.3 Time evolution of nanobubbles . . . . . . . . 80

5.3.4 Correlation between global current and local nanobubble growth . . . . . . . . . . . . . 82

5.3.5 NaCl solution as electrolyte . . . . . . . . . . 89

5.4 Conclusions . . . . . . . . . . . . . . . . 92

References ....................... 95

6 Conclusion and Outlook 97

6.1 General Conclusions . . . . . . . . . . . . . . . 97

6.2 Outlook ....................... 100

References ..................... 102

$\begin{array}{ll}\text { Summary } & 103\end{array}$

$\begin{array}{ll}\text { Samenvatting } & 107\end{array}$

$\begin{array}{ll}\text { Acknowledgements } & 111\end{array}$ 
About The Author

113 


\section{1 \\ Introduction}

\subsection{General Introduction}

Over the last decade, numerous experiments revealed the existence of nanoscopic soft domains at the liquid-solid interfaces, see [1-11] and references therein. The most consistent interpretation of the experimental results is that these soft domains are surface nanobubbles, i.e., nanosized gas bubbles located at liquid-solid interface, see Figure 1.1 and 1.2 for examples. To address several early observations: Tyrrell et al. showed that hydrophobic surfaces in water, imaged with tapping mode atomic force microscope (AFM), reveal to be covered with nanobubbles that are close packed and irregular in cross section; the nanobubbles have a radius of curvature of the order of $100 \mathrm{~nm}$ and a height of 20-30 nm [1]. Later, Holmberg and coworkers claimed that nanobubbles seem to form spontaneously when gold surfaces are immersed in clean water and argued that it is probably a general phenomenon at water-solid interfaces [4]. In 2004, Simonsen et al. showed that nanbubbles of decreasing size and number, associated at solid-liquid interface, are observed as on a hydrophobic surface as the hydrophobicity of the subphase increases; the disturbance of the water structure in the contact region induces the formation of nanobubbbles [3]. In the same year, Zhang et al. summarized that 
the dissolved gas in the liquids is essential for the formation of nanobubbles at mica-water interface and the effect of liquid temperature is significant [8].
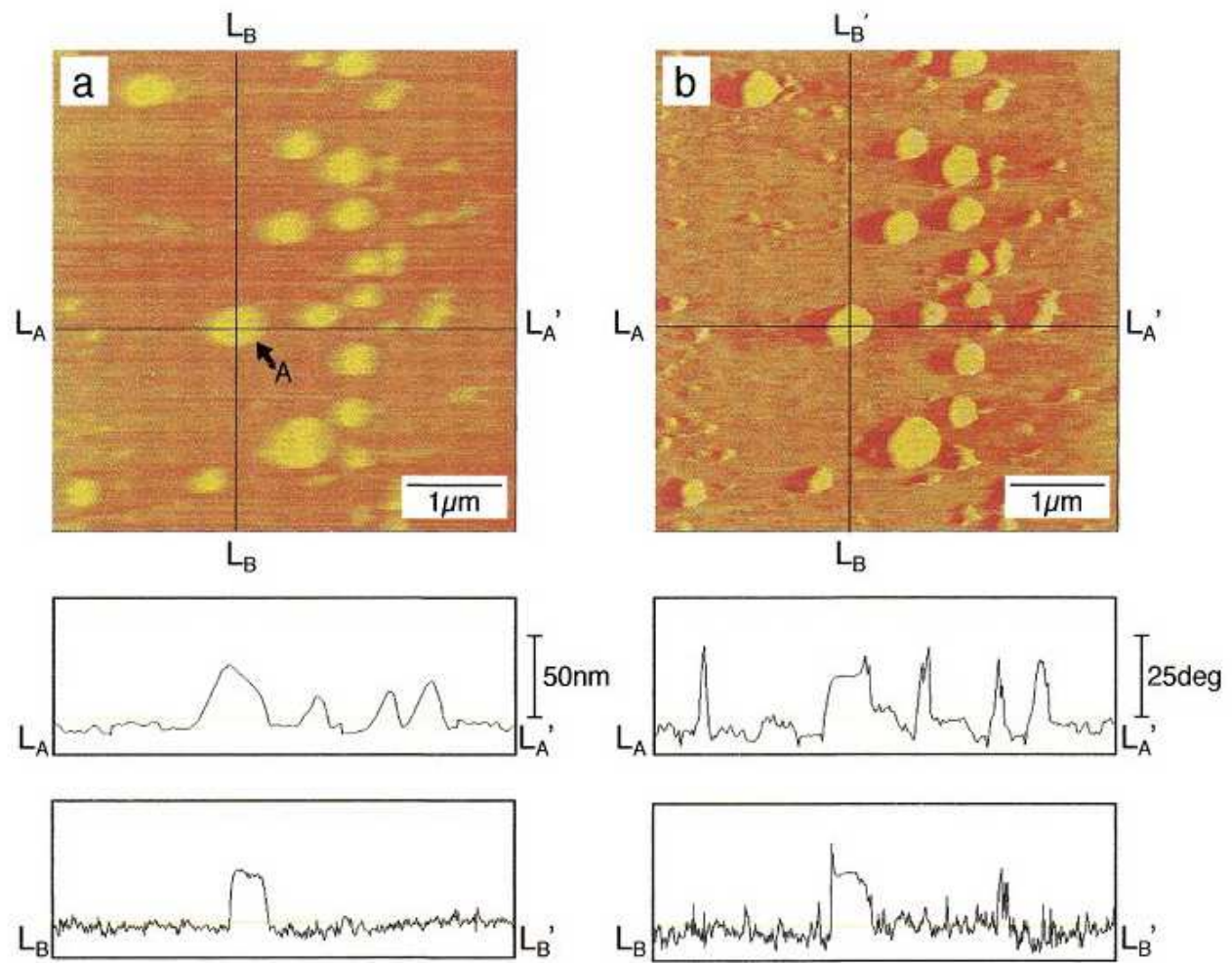

Figure 1.1: AFM height image (a) and phase image (b) of nanobubbles, showing cross-sectional views of a nanobubble which is $40 \mathrm{~nm}$ high with a $25^{\circ}$ phase shift from the surface. Adopted from [2].

As we can see from the previous studies, most experiments employ AFM [1-9]. Tapping mode of AFM is absolutely prevailed, although detecting nanobubbles with AFM contact mode has also been reported [4]. Tapping mode is a key advance in AFM. This potent technique allows nanoscale high-resolution topographic imaging of surfaces that are easily damaged or loosely hold to their substrate, such as surface nanobubbles. Tapping mode provides high material (chemical) sensitivity and overcomes problems associated with friction, adhesion, electrostatic forces, and other difficulties at surfaces. In addition, the AFM probe is capable to oper- 
ate in both air and liquid ambient, which is essential for the imaging of nanobubbles. However, as complement, other techniques such as rapid cryofixation-freeze fracture [10] and neutron reflectometry [11] have been used to investigate nanobubbles as well.
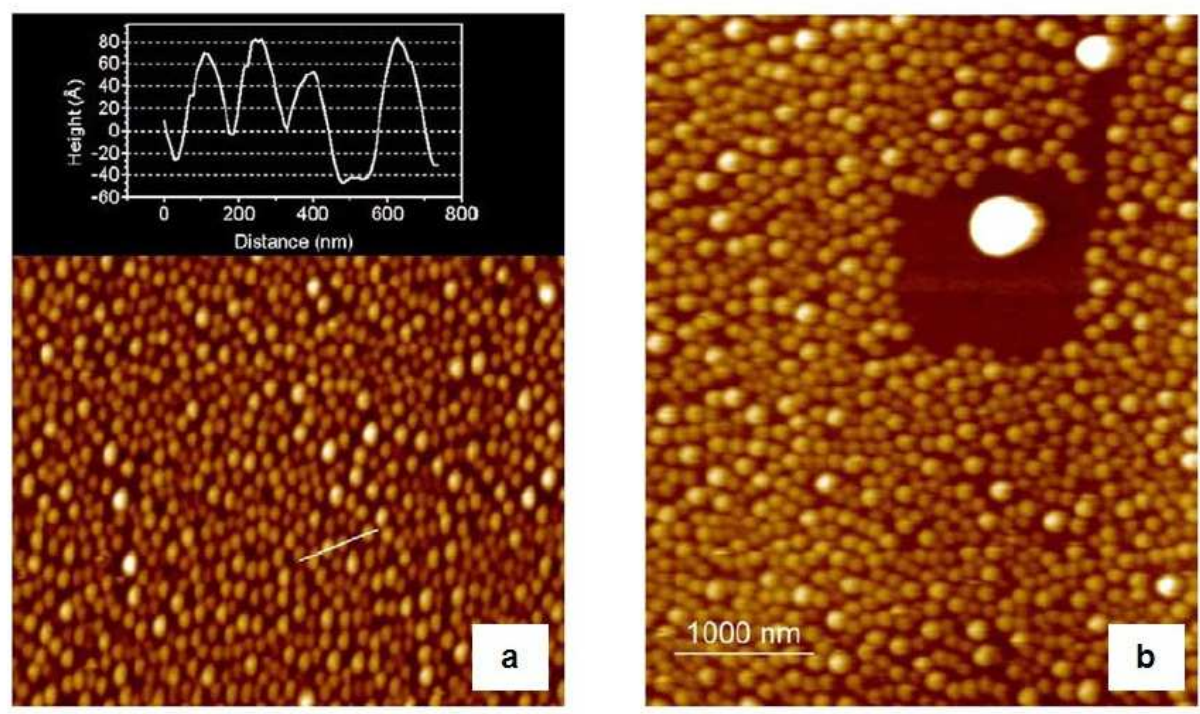

Figure 1.2: AFM images of nanobubbles spontaneously formed by immersion of the polystyrene surface in ultra pure water. The bubbles are covering around 60 percent of the surface area. By scanning a small region with increased tapping amplitude, the bubbles in this region are fused to one big bubble, as shown in (b). Adopted from [3].

In previous studies, the solid substrates used are various, including gold [4], silane-hydrophobilized silicon wafer $[9,10]$, polystyrene $[3,5]$, mica [8], highly oriented pyrolytic graphite (HOPG) [6,7], and bare silicon (with a native oxide layer) [9], which however all provide flat surfaces on nanoscale. Although it is not a necessary condition to form nanobubbles, such a flat surface does help to identify nanobubbles from the surface background. As liquid, highly purified water (Milli-Q) is most commonly used, though some experiments were done with alcohols [3] or dilute sulfuric acid solutions [7] as a comparison to water.

Nanobubbles, as observed in previous studies, resemble spherical caps with a height of the order of $10 \mathrm{~nm}$ and a diameter of the order of $100 \mathrm{~nm}$. 
Nanobubbles are claimed to consist of gas - this is supported, for instance, by the fact that nanobubbles can be merged by the tip of an AFM to form a larger bubble as shown in Figure 1.2 [3], or by the fact that they disappear upon degassing of the liquid $[7,8,10]$, see Figure 1.3. Nanobubbles can be manipulated to disappear from contact mode AFM images and then to reappear by changing the scanning force [4]. However, once they have formed, the nanobubbles remain very stable, at least for several hours. Experimental observations show that nanobubbles mostly appear on hydrophobic surfaces (contact angle $>90^{\circ}$ ) in water. Nevertheless, it has been demonstrated that even on hydrophilic surfaces (contact angle $<$ $90^{\circ}$ ) nanobubbles can efficiently form if the surface is initially covered by ethanol which is subsequently replaced by water (the so-called ethanolwater-exchange process, see reference [6]). Also, it has been suggested that the majority of nanobubbles prefer to form in the vicinity of hydrophobic patterns on a hydrophilic surface [5]. These observations lead to the conclusion that hydrophobic surfaces favor the formation of nanobubbles. In addition, the nanoscopic contact angle of the nanobubbles (typically $\sim 170^{\circ}$ ) is much larger than the macroscopic contact angle on the surface (typically $\sim 100^{\circ}$ ) $[3,6]$. Following those observations, we conclude that nanobubbles are sensitive to liquid conditions and surface properties. The extraordinary shape of the nanobubbles, the so-called pancake appearance, which is connected to their remarkably large nanoscopic contact angles, is one of the most critical arguments on the formation of those nanoscopic surface bubbles.

\subsection{Nanobubbles Are Puzzling}

Why do nanobubbles attract so much scientific interest? There are two main reasons.

First, on the application side, and specifically in the field of micro- and nanofluidics, nanobubbles are a potential candidate to explain a number of phenomena associated with liquid-solid interfaces. We address two of them: (i) The attractive force in the range of $10-100 \mathrm{~nm}$ observed between two hydrophobic surfaces in solutions: Studies suggest that nanobubbles can bridge two opposing surfaces, attracting them towards each other $[1,12,14]$. As Tyrrell and coworkers demonstrated, complementary force measurements show that nanobubbles present the long range hydrophobic attraction, including a jump into a soft contact and a pre- 

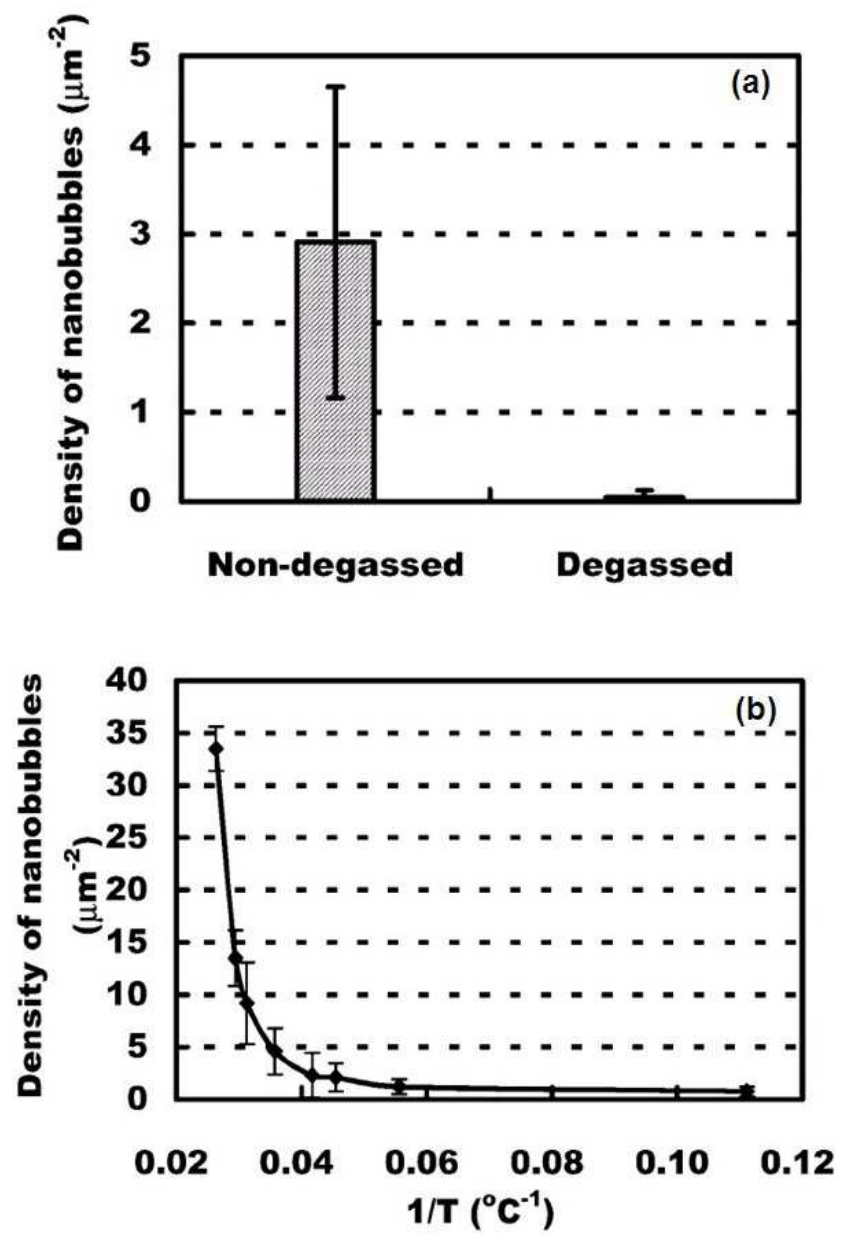

Figure 1.3: (a) shows the effect of dissolved gas on the formation of nanobubbles. The average density of nanobubbles decreases obviously when the liquid is degassed. (b) shows the effect of liquid temperature on the formation of nanobubbles. The number of nanobubbles per micron increases with the liquid temperature and shows a rapid growth when the temperature is higher than $30^{\circ} \mathrm{C}$. Adopted from [8]. 

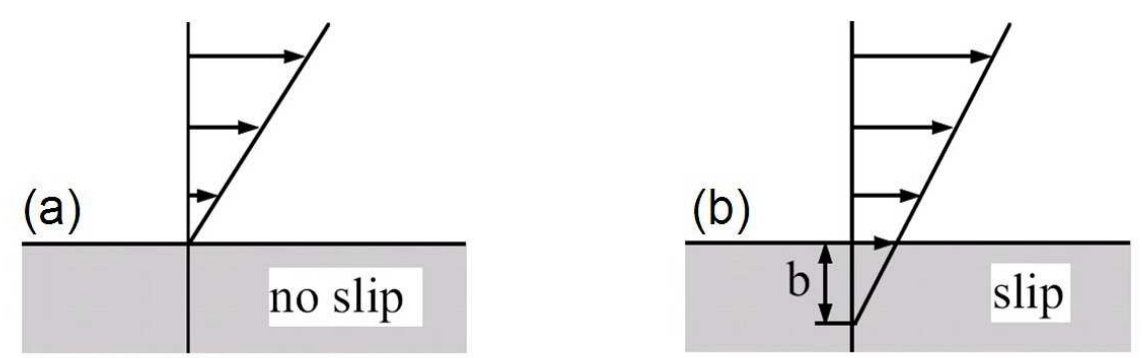

Figure 1.4: Velocity profile close to a wall depending on the boundary condition: (a) zero velocity at the wall and (b) finite velocity characterized by a slip length $b$.

jump repulsion. The distance of the jump is correlated with the height of the nanobubbles [13]. (ii) The liquid slippage at wall: The standard boundary condition for fluid flow along a wall is the no-slip condition. However, a deviation (slip condition) from the dogmatic no-slip hydrodynamic boundary condition is observed in numerous experiments, see Figure 1.4. For example, with water flowing in thin hydrophobic capillaries, there are some early qualitative evidences for slippage $[15,16]$, and calculations show that the first layer of water molecules is depleted in the presence of a hydrophobic wall [17]. The slip condition is crucial for fluidic systems when the liquid-volume over surface-dimension ratio is small. It has been suggested that the presence of nanobubbles sitting on hydrophobic surfaces significantly promotes slip because nanobubbles provide a quasi zero shear stress boundary condition leading to an average reduction of the liquid friction on the walls, a wanted phenomenon to reduce hydrodynamic resistance [18-21].

Second, more fundamentally, nanobubbles should not exist. According to the experimental data these bubbles have a radius of curvature $\mathrm{R}$ smaller than $1 \mu \mathrm{m}$, and therefore they should dissolve on timescales far below a second [22,23], due to a large Laplace pressure inside of the bubbles, which in a bubble, with radius of e.g. $200 \mathrm{~nm}$ amounts to approximately $5 \mathrm{~atm}$. In marked contrast the experiments reveal that nanobubbles are stable for periods as long as a few hours. The fundamental principle of the surprising stability of nanobubbles is still a mystery. Moreover, the nanoscopic contact angle, extracted from the AFM height profiles of nanobubbles, is much larger than the macroscopic contact an- 
gle obtained from standard contact angle measurements $[3,6]$. Is there a transition of contact angle from macroscale to nanoscale on the same surface? Generally, apart from convincing experimental evidence for the existence and stability of nanobubbles, fairly little is known and puzzling questions still remain yet to be answered.

In addition, nanobubbles have broad technical applications. In order to characterize the physical properties of nanobubbles, We focus on the control of their appearance, density, location and shape. This consequently will help to uncover the big mysteries of those tiny bubbles and will bring us benefits in developing novel technologies of, for example, inkjet printing, medicine delivery, biological fluidic systems, and much more.

\subsection{Guide through The Chapters}

The purpose of this Thesis is to elucidate nanobubble-related issues by performing AFM measurments of nanobubbles under various conditions. A number of experiments are designed and performed to demonstrate the effects of substrate surface and liquid on the density and size of nanobubbles. Also, it is found that the formation of nanobubbles responds to the variation of hydrophobicity on an HOPG surface. Finally, we demonstrate that electrolysis of water is a reliable route to control the appearance and density of nanobubbles.

AFM tapping mode is the key technique in the study of nanobubbles. AFM initially was used to image dry surfaces, providing high quality images of surface structures with high resolution (atomic resolution is achievable). Over the last years, AFM has been increasingly operated in wet environments, mainly due to the requirements from biology laboratories where AFM is capable to carry out tasks of imaging surfaces in wet condition, offering nanoscopic resolution with certain chemical, temperature, and electrical tolerances. Therefore, AFM tapping mode became the primary experimental method immediately after the surface nanobubble issues appeared. No other techniques are able to allow experimentalists to study nanobubbles in such a manipulatable way as AFM is. However, there are still ongoing studies and debates in understanding AFM operation in liquid environment. Also, because of the small size of nanobubbbles, the information revealed by AFM is circumstantial and indirect, and 
the AFM imaging probe interacts with the nanobubbles. Therefore, appropriate understanding and operating of AFM are of key importance in the study of nanobubbles. In Chapter 2, we introduce the working principle of AFM, the operation modes, and the advantages as well as the limitations of AFM in surface studies. We show what surface information AFM provides, in terms of topography, phase, and force curves. Finally, we concisely address the current development of AFM and emphasize its applications in liquids.

In Chapter 3, the effects of the substrate surface on nanobubbles are discussed. Among the different substrates we tested, namely, hydrophobic or hydrophilic coated gold, bare silicon (with native oxidation layer), mica, and hydrophobilized silicon wafer with different coating agents, the hydrophobic silanated silicon wafer is chosen for further study. This is due to the fact that hydrophobic surfaces favor nanobubble formation and the preparation method of these surfaces is well known. On the surface, nanobubble stability is examined: they remain stable on timescale of hours. A link between surface roughness and nanobubbles is suggested: the size of the nanobubbles is in the same scale as surface roughness. However, later studies show that nanobubbles can form anywhere on the surface though with a preference to form at rougher locations, such as nanometer grooves resulting from the sample polishing process. To show the gas nature of nanobubbles, a direct comparison between nanobubbles and microcavities is established: image nanobubbles next with a microcavity on the surface which has been proved to trap gas - that actually is a manufactured microbubble in the vicinity of nanobubbles. We also show that nanobubbles can move along nanogrooves on the surface due to the influence of scanning AFM tip. Increasing the substrate temperature forms nanobubbles in situ, thus the birth and growth of nanobubbles can be observed. Moreover, creating nanobubbles in situ allows to investigate the surface topography underneath the nanoubbbles. Furthermore, we show that an alcohol prewash of the surface affects the nanobubble formation with regard to nanobubble density and size.

The effect of liquids on nanobubbles is also discussed in Chapter 3, in terms of water temperature, gas concentration, surfactant added in, and the so-called ethanol-water-exchange process. Increasing the water temperature facilitates the formation of nanobubbles because the increase of water temperature effectively leads to a supersaturation of gas in the water. Also, we see that nanobubbles do not disappear when the water cools down to ambient conditions. To further support that an increase of gas 
concentration of water enhances nanobubble formation, we pressurized the water with $\mathrm{CO}_{2}$ at different pressures - corresponding to different gas concentrations in water. We see that the nanobubble density is higher and the nanobubble size is smaller at higher gas concentration. Adding butanol drops causes a decrease of radius of curvature of the nanobubbles. Another method to dramatically enhance nanobubble formation is the ethanol-water-exchange process, i.e., the surface is initially covered by ethanol which is subsequently replaced by water.

Applying the ethanol-water-exchange process, nanobubbles efficiently form on highly orientated pyrolytic graphite (HOPG) surfaces. In Chapter 4 , We show that the distribution of nanobubbles is inhomogeneous on the (under-water) surface of HOPG, reflecting the presence of atomic steps: The formation of nanobubbles is strongly enhanced at the upper side of the atomic steps, i.e., the most hydrophobic area on the surface. In contrast, no nanobubbles are formed at the lower side of the steps, i.e., the most hydrophilic area. The width of this nanobubbble-free zone is approximately $20 \mathrm{~nm}$. We thus establish a correlation between surface topography and nanobubble formation. In addition, we show that the profile of nanobubbles is sensitive to the applied AFM tip-force, demonstrating the deformability of nanobubbles. As comparison, similar measurements are carried out on a solid object and a meniscus in microcavity.

Chapter 5 describes the work motivated by the fact that electrolysis of water is a reliable means to rapidly produce a high local gas concentration at the electrode surfaces, as well as by the observation that gas concentration significantly affects the formation of nanobubbles. Electrolysis of water therefore can be a steady method that leads to control of the appearance and growth of nanobubbles. This is demonstrated by performing AFM measurements of nanobubbles on HOPG surfaces. The HOPG surface acts as one of the electrodes. We show that both oxygen (at anode) and hydrogen (at cathode) nanobubbles are produced by electrolysis of water, with varied bubble- coverage, volume, and size at different voltage. In this Chapter, we present the real-time process of nanobubbles and the electric current that flows from one electrode to the other. Interestingly, a correlation between the nanobubble development and the current decay is found. Based on the observations, we suggest how electrolytic gas emerges on the surface and a possible mechanism in which nanobubbles remain stable. To test the repeatability, sodium chloride solution is used as electrolyte. Similar observations are obtained as compared to the pure water case. 
The work presented in this Thesis is an experimental study to characterize the physical properties of nanobubbles, focusing on how surface properties and liquid conditions influence the appearance, size, density, and shape of nanobubbles, which is all concluded in Chapter 6. 


\section{References}

[1] Tyrrell, J. W. G.; Attard, P. Phys. Rev. Lett. 2001, 87, 176104.

[2] Ishida, N.; Inoue, T.; Miyahara, M.; Higoashitani, K. Langmuir 2000, $16,6377$.

[3] Simonsen, A.C.; Hansen, P.L.; Klösgen, B. J. Colloid Interface Sci. 2004, 273, 291.

[4] Holmberg, M.; Kühle, A.; Garnæs, J.; Mørch, K. A.; Boisen, A. Langmuir 2003, 19, 10510.

[5] Agrawal, A.; Park, J.; Ryu, D. Y.; Hammond, P. T.; Russel, T. P.; McKinley, G. H. Nano Lett. 2005, 5, 1751.

[6] Zhang, X. H.; Maeda, N.; Craig, V. S. J. Langmuir 2006, 22, 5025.

[7] Zhang, L.; Zhang, Y.; Zhang, X.; Li, Z.; Shen, G.; Ye, M.; Fan, C.; Fang, H.; Hu, J. Langmuir 2006, 22, 8109.

[8] Zhang, X. H.; Zhang, X. D.; Lou, S. T.; Zhang, Z. X.; Sun, J. L.; Hu, J. Langmuir 2004, 20, 3813.

[9] Agrawal, A.; McKinley, G. H. Mater. Res. Soc. Symp. Proc. 2006, 899E.

[10] Switkes, M.; Ruberti, J. W. Appl. Phys. Lett. 2004,84, 4759.

[11] Steitz, R.; Gutberlet, T.; Hauss, T.; Klösgen, B.; Krastev, R.; Schemmel, S.; Simonsen, A. C.; Findenegg, G. H. Langmuir 2003, 19, 2409.

[12] Parker, J. L.; Claesson, P. M.; Attard, P. J. Phys. Chem. 1994, 98, 8468.

[13] Tyrrell, J. W. G.; Attard, P. Langmuire 2002, 18, 160.

[14] Attard, P. Langmuir 1996, 12, 1693.

[15] Churaev, N.; Sobolev, V.; Somov, A. J. Colloid Sci. 1984, 97, 574.

[16] Blake, T. Colloids Surf. 1990, 47, 135.

[17] Barrat, J. L.; Bocquet, L. Phys. Rev. Lett. 1999, 82, 4671.

[18] Bonaccurso, E.; Butt, H. J.; Craig, V. S. J. Phys. Rev. Lett. 2003, 90, 144501. 
[19] Lauga, E.; Brenner, M. P.; Stone H. A.; in Handbook of Experimental Fluid Dynamics, edited by Tropea, C.; Foss, J.; Yarin A.,Springer, New York 2005.

[20] Neto, C.; Evans, D. R.; Bonaccurso, E.; Butt, H. J.; Craig, V. S. J. Rep. Prog. Phys. 2005, 68, 2859.

[21] de Gennes, P. G. Langmuir 2002, 18, 3413.

[22] Epstein, P. S.; Plesset, M. S. J. Chem. Phys. 1950, 18, 1505.

[23] Ljunggren, S.; Eriksson, J. C. Colloids Surf. A 1997, 129, 151. 


\section{2 \\ Experimental Instrument: Atomic Force Microscope}

\subsection{Introduction}

Atomic Force Microscope (AFM) has been rapidly developed into a powerful and versatile technique to solve processing and material problems in aerospace, automotive, biological, chemical, electronics, telecommunications, and energy industries, since its invention in 1986 by G. Binnig, C. F. Quate, and Ch. Gerber [1]. Like all other scanning probe microscopes, AFM utilizes a sharp tip moving over the surface of a sample in a raster scan. The AFM tip is on the end of a cantilever which bends in response to the force between the tip and the sample surface.

The first AFM cantilever was made by a tiny shard of diamond glued onto the end of a tiny strip of gold foil. In the fall of 1985, Binnig and Gerber used the cantilever to examine insulating surfaces. The cantilever was pressed against the sample surface while the sample was scanned directly underneath the tip. The force between tip and sample was measured in such a way: deflection of the cantilever due to the surface was tracked by monitoring the tunneling current through a second metal tip positioned 
above the cantilever. Thus the current signal was translated back as the surface contour. A resolution of $30 \mathrm{~nm}$ was achieved. This was how the very first AFM delineated surfaces. Later on, microfabricated silicon cantilevers were introduced. This breakthrough in cantilever manufacture very practically and dramatically improved AFM imaging quality.

After several years later more advanced microcantilever as well as optical detectors have been developed, AFM - this new tool for surface science - has been embraced by scientists and technologists. Figure 2.1 shows the PicoSPM AFM in our laboratory. Today AFM cantilevers made of Si or $\mathrm{Si}_{3} \mathrm{~N}_{4}$ with a tip extending down from the end of the cantilever are broadly in use, with which AFM users achieved many outstanding results on molecular and atomic scales. An example of modern cantilevers is shown in Figure 2.2. AFM allows scientists and technologists not only to image surfaces with atomic resolution, but also to measure forces on nanonewton scale. AFM is capable to investigate many kinds of materials including biological membranes, ceramics, composites, glasses, metals, polymers, semiconductors, synthetic materials, and thin films. AFM is involved in studies of different phenomena such as abrasion, adhesion, cleaning, etching, friction, lubrication, plating, polishing, and many more.

\subsection{Working Principle of AFM}

The principles on how the AFM works are very simple. An atomically sharp tip scans over a surface at such a position that there are force interactions between the tip and the surface. The feedback mechanisms enables the electric piezo scanners to maintain the tip either at a constant force (to obtain height information), or at a constant height (to obtain force information) above the surface. An optical system is applied to detect the vertical motion of the tip due to the surface topography. The optical system contains a photodetector and a diode laser that is focused onto the back of the cantilever. As the tip scans the surface of the sample, moving up and down with the contour of the surface, the laser beam is deflected off the cantilever onto the photodetector. The photodetector, which has dual (upper and lower) element photodiodes, measures the difference in light intensities between the photodiodes, and then converts it to voltage. Feedback from the photodiode difference signal, through the software control from a computer, enables the tip to maintain either a 


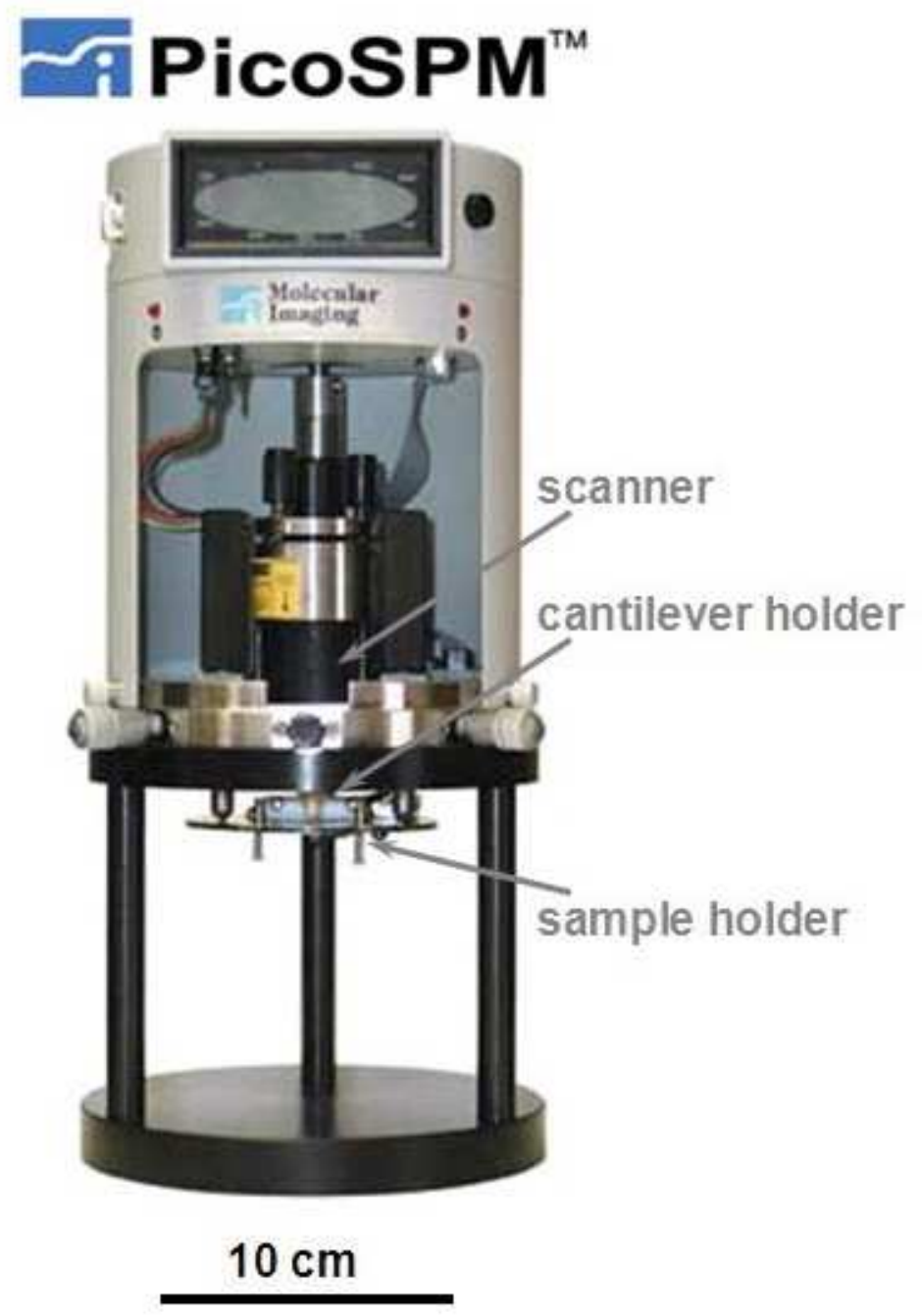

Figure 2.1: The PicoSPM Atomic Force Microscope in our laboratory. 


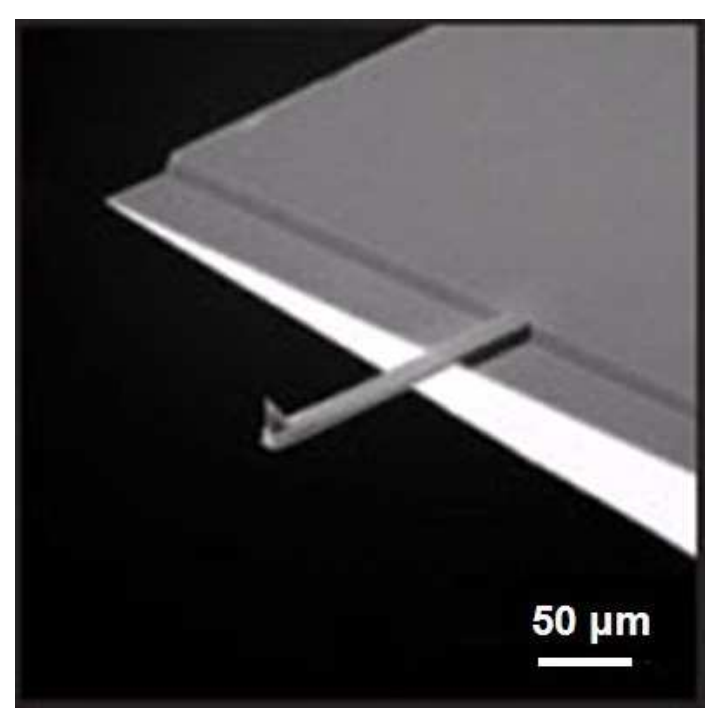

Figure 2.2: SEM image of a commercial AFM cantilever made of $\mathrm{Si}_{3} \mathrm{~N}_{4}$ with a tip extending from the end of the cantilever. Adopted from MikroMasch AFM Probes.

constant force or a constant height above the surface. Figure 2.3 schematically shows the process.

The primary purpose of AFM is to quantitatively measure the surface structure with a resolution down to atomic scales on various types of surfaces. AFM scanners are designed to translate either the sample under the cantilever or the cantilever over the sample. By scanning in either way, the local height of the surface structure is measured. Three-dimensional topographical maps of the surface are then constructed by plotting the local surface height versus horizontal AFM tip position. In addition, local force changes between the tip and the surface can be measured. These forces depend, among others, on the chemical properties of the surface.

\subsection{The Standard Operation Modes}

\subsubsection{Contact mode}

Contact mode is the most commonly used AFM operating mode. In contact mode, the tip scans the surface in a close contact. The force on the 


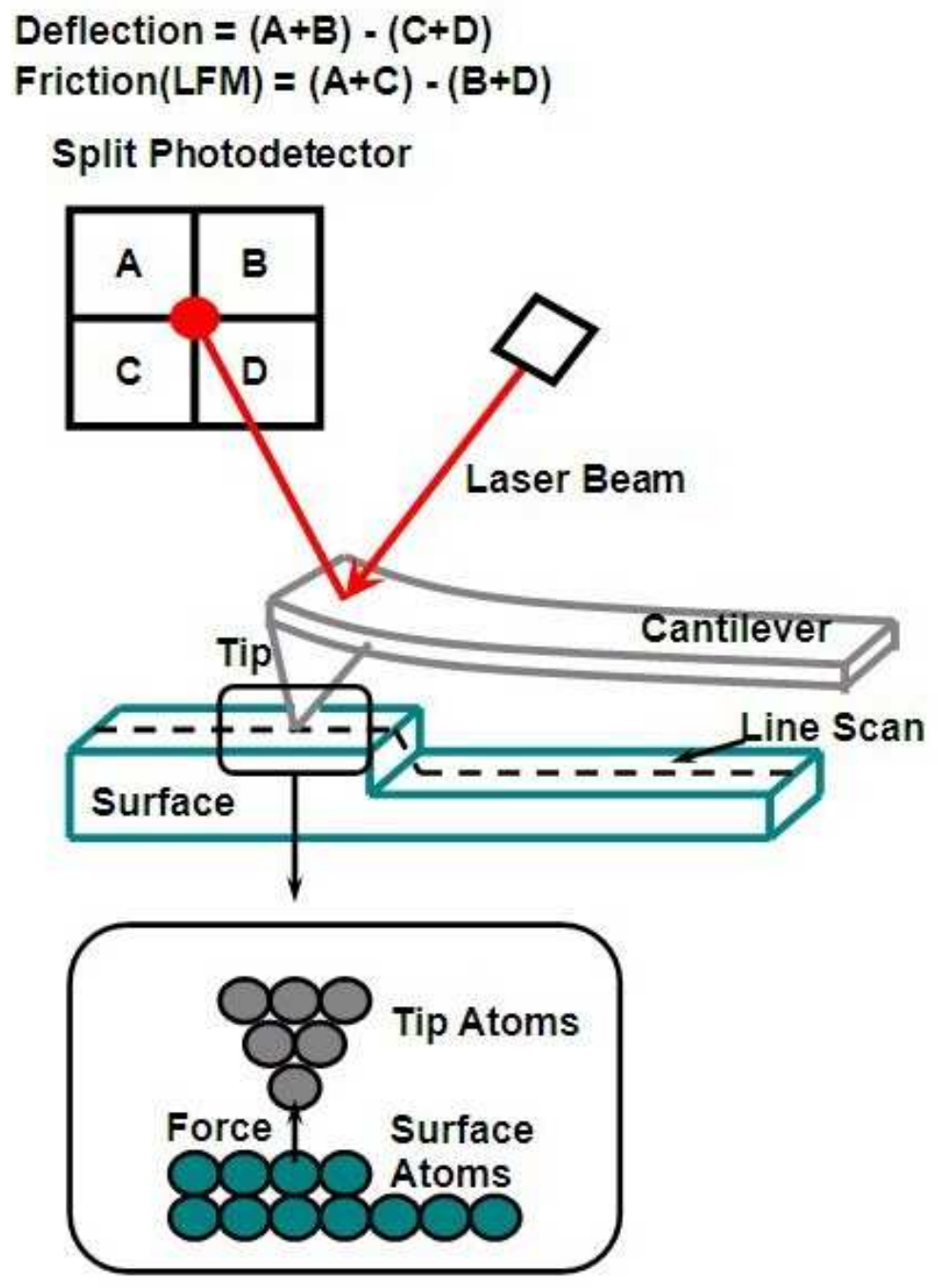

Figure 2.3: Sketch describing the AFM working principle. An atomically sharp tip scans over a surface. The tip surface force changes due to the surface topography are carefully revealed by the (vertical) motion of the cantilever, which is measured by an optical detecting system. The photodetector then translates the optical signal to an electronic signal in order to construct a topography image of the surface. 


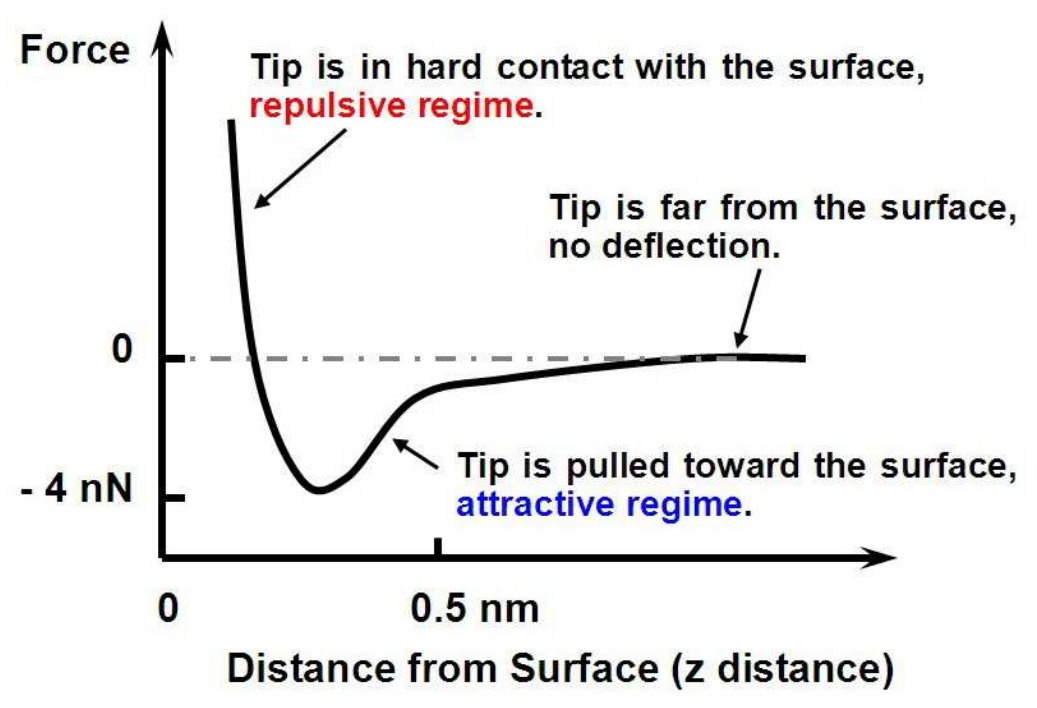

Figure 2.4: As AFM tip is brought close to a sample surface, the interaction force between the tip apex atom and the surface atoms behaves as depicted in Figure 2.3. The behavior of the force as a function of the distance to the surface is described in the plot. Attractive or repulsive force alternatively occurs depending on the distance. Note that the attractive force is weaker as compared to the repulsive force.

tip is repulsive with a mean value in the nanonewton range, see Figure 2.4. This force is triggered by pushing the cantilever against the sample surface with an electric piezo element. The deflection of the cantilever is detected and compared to a preset value of the deflection in a feedback loop. During scan when the measured deflection changes from the preset value due to the surface structures, the feedback loop sends a voltage to the piezo element to raise or lower the sample to restore the preset value of deflection. The voltage that the feedback amplifier applies to the piezo is a measure of height of the features on the sample surface. It is displayed as a function of the horizontal position of the surface. Thus it delineates surface structures. The majority of contact mode AFM operates in ambient atmosphere or in liquids, although some operate in ultrahigh vacuum (UHV) as well.

However, there are some problems with contact mode, which are mainly caused by excessive tracking forces applied by the AFM tip to the sample surface. The impact can be reduced by minimizing the force, but this is 
practically limited by the magnitude of the force that can be controlled by the users during operation in ambient environments. Under ambient conditions, sample surfaces are covered by a layer of adsorbed gases consisting primarily of water vapor and nitrogen. The layer forms a meniscus with the tip and pulls the tip with a force caused by surface tension. This force is in the order of 100 nanonewtons. In addition, a large class of samples, including semiconductors and insulators, can trap electrostatic charges (partially dissipated and screened in liquid). These charges contribute to additional substantial attractive forces between AFM tip and sample surface. All of these forces combine to define a minimum normal force. This normal force creates substantial frictional forces as the tip scans over the surface. In practice, it appears that these frictional forces are far more destructive than the normal force and can damage the surface, spoil the cantilever, and distort the resulting data. An attempt to avoid these problems is the non-contact mode AFM.

\subsubsection{Non-contact mode}

The introducing of non-contact mode received a warm welcome by surface scientists. Non-contact mode is used to avoid situations where repulsive forces between tip and surface may ruin the measurements. In this mode the AFM tip hovers above the sample surface. The attractive forces, chiefly van der Waals force between the tip apex atom and sample surface atom, dominate the tip-surface interaction, see Figure 2.4. Unfortunately, the attractive forces are substantially weaker than the repulsive forces used by the contact mode. The tip therefore must be given a small oscillation so that AC detection methods can be used to detect the small forces between the tip and the sample by measuring the change in amplitude, phase, or frequency of the oscillating cantilever in response to force gradients from the sample $[2,3]$. The cantilever is allowed to vibrate in a very small amplitude (below $1 \mathrm{~nm}$ ). Thus topographic images are constructed by scanning the tip above the surface. For highest resolution, it is necessary to measure force gradients from van der Waals forces which may extend only a nanometer from the sample surface. In general, the fluid contaminant layer is substantially thicker than the range of the van der Waals force gradient. Consequently, attempts to image the true surface with non-contact AFM fail as the oscillating cantilever becomes trapped in the fluid layer or hovers beyond the effective range of the forces it attempts to measure. As a solution, the tapping mode was introduced. 


\subsubsection{Tapping mode}

A few years later after the introducing of non-contact mode, two major modifications were proposed [4]. First, oscillation amplitudes were enhanced up to $100 \mathrm{~nm}$. Second, stiffer cantilevers are adopted. Adding those two values avoids the trapping of tip by surface forces. This method is coined as tapping mode (also called as intermittent contact mode). Tapping mode is a key advance in AFM. This potent technique allows to obtain high-resolution topography images of surfaces that are easily damaged or loosely held to their substrate. Tapping mode alternately places the tip in contact with the surface to provide high resolution and then lifts the tip off the surface to avoid dragging the tip across the surface. In such a way, tapping mode overcomes problems associated with friction, adhesion, electrostatic forces, and so on. During a tapping mode scan, the cantilever oscillates at or near its resonant frequency by an electric piezo element. The oscillating amplitude is rather high compared to the non-contact mode. When the tip is not in contact with the surface, the typical amplitude is greater than $20 \mathrm{~nm}$ (free amplitude). The oscillating tip is then moved toward the surface until it begins to lightly touch, or tap the surface, causing now the cantilever to have a reduced amplitude (set-point amplitude). During scanning, the vertically oscillating tip alternately contacts the surface and lifts off, generally at a frequency of 50 - $500 \mathrm{kHz}$ (in ambient air). As the oscillating tip intermittently contacts with the surface features, the cantilever oscillation is necessarily reduced due to energy loss caused by the tip contacting the surface. The reduction in oscillation amplitude is adopted to identify and measure surface features.

During tapping mode operation, the cantilever oscillation amplitudes are maintained constant (set-point amplitude) by a feedback loop. When the tip passes over a bump on the surface, the cantilever has less freedom to oscillate and the amplitude of the oscillation decreases. Conversely, when the tip passes over a cavity, the cantilever has more freedom to oscillate and the amplitude increases. The oscillation amplitude of the cantilever is measured by a photodetector. The signals are sent to a feedback system which forces the piezo to move the cantilever up (or down) to increase (or decrease) oscillation amplitude back to the set-point value. This vertical motion of the cantilever is recorded and plotted versus the horizontal position to construct a three-dimensional topography image of the surface. 
When the tip taps the surface, the high oscillation frequency (50 - 500 $\mathrm{kHz})$ and large oscillation amplitude (10 - $100 \mathrm{~nm})$ effectively reduce the tip-sample adhesion forces. Unlike contact and non-contact modes, when the tip contacts the surface in tapping mode it has sufficient oscillation amplitude to overcome the tip-sample adhesion forces. Also, the surface material is not pulled sideways by shear forces since the applied force is always vertical. Tapping mode inherently prevents the tip from causing damages or sticking to the surface during scanning. Another advantage of the tapping mode technique is its large, linear operating range, which makes the vertical feedback system very stable, allowing routinereproducible surface measurements. Tapping mode AFM has been developed as a reliable method to achieve high resolution without inducing destructive frictional forces both in air and in liquid. With the tapping mode technique, the very soft and fragile samples can be imaged successfully. Also, incorporated with phase imaging, the tapping mode AFM can be used to analyze the materials (chemical) properties on the surface.

We have to conclude that AFM is a unique surface study method with following most outstanding characteristics: true atomic resolution, threedimensional measurements of atomic forces, observation of insulators, control of atomic forces, measurement of mechanical response, mechanical manipulation of individual atoms, and mechanical assembly atom by atom [5].

\subsection{What Beyond Topographic Imaging}

\subsubsection{Phase imaging}

Phase imaging is a powerful extension of tapping mode AFM. It provides nanometer-dimensional information about the surface structure that is often not revealed by other scanning probe microscopes (SPM). By mapping the phase of the cantilever oscillation during scanning, phase imaging goes beyond simple topographic delineation of the surface. It detects variations in adhesion composition, friction, viscoelasticity, and other more properties. Phase imaging serves a broad range of applications including identification of contaminants, mapping of surface friction, mapping of different components in composite materials, and differentiating regions of high and low surface adhesion or hardness. In many cases, phase imag- 
ing often provides surface information more rapidly and with higher resolution, complementing lateral force microscopy (LFM) and force modulation techniques. Phase imaging is as fast and as easy to use as topographic imaging, with all its benefits for imaging soft, adhesive, easily damaged or loosely bonded samples. While measuring, both topography and phase images are viewed simultaneously in real time. The resolution of phase imaging is comparable to the one of topography.

In tapping mode AFM, the cantilever is excited into resonant oscillation with an electric piezo element. The oscillation amplitude is used as a feedback signal to measure topographic contrasts of the sample surface. In phase imaging, the phase lag of the cantilever oscillation, caused by the variations in material properties on sample surface such as adhesion and viscoelasticity, is monitored and used to build the phase image. Phase imaging is a powerful tool for mapping variations in material properties at very high resolution. It can be turned on while topographic imaging without any cost in speed or resolution. Phase imaging promises to play an important role in the study of material properties at the nanometer scale.

\subsubsection{Force curves}

In addition to the topography and phase measurements, AFM also provides information of the amount of force encountered by the cantilever as the cantilever is brought close to and even indented into a sample surface, and then pulled away. This technique is used to measure the longrange attractive or repulsive forces between the cantilever and the sample surface, elucidating local chemical and mechanical properties such as adhesion and elasticity, and even thickness of adsorbed molecular layers or bond rupture lengths. Force curves typically show the deflection of the free end of the cantilever as the fixed end of the cantilever is brought vertically towards and then away from the sample surface. This deflection is plotted at many points along the journey of the cantilever, thus to form a force-distance curve.

During a force recording, the AFM tip starts at such a position that there is no contacting with the surface. In this region, if the tip senses a long-range attractive (or repulsive) force cantilever will bend downwards (or upwards). As the tip is brought close to the surface, it may jump into contact if it feels sufficient attractive force from the sample surface. Once the tip is in contact with the surface, the cantilever deflection will increase 
as the fixed end of the cantilever is still being brought closer to the surface. If the cantilever is sufficiently stiff, the tip may indent into the surface at this point. In this case, the slope of the contact part of the force curve can provide information about the elasticity of the sample surface. After loading the cantilever to a desired force value, the process is reversed. As the cantilever is withdrawn, adhesion or bonds formed during contact with the surface may cause the tip to adhere to the sample some distance away from the initial contact point. As the cantilever is brought further away, the key measurement of the AFM force curve comes when the adhesion is broken and the cantilever becomes free from the surface. This is used to measure the rupture force required to break the bond or adhesion.

The study of fundamental interactions between surfaces has attracted tremendous attentions across physics, chemistry, materials science and a variety of other disciplines. With a force-sensitivity down to a few piconewtons, AFM is an excellent tool to investigate these fundamental forces. AFM has made its mark on a wide variety of applications as a topographic measurement and mapping tool. Currently AFM force measurements are providing information on atomic and molecular scale interactions such as adhesive and elastic response. With these measurements, we are beginning to revolutionize the ways that we used to quantitatively observe and think about the physical and chemical world.

\subsection{Comparisons with Other Microscopes}

1), AFM versus STM: Scanning Tunneling Microscope (STM) is the precursor of AFM. The resolution of STM is casually better than AFM because of the exponential dependence of the tunneling current on distance. The force-distance dependence in AFM is much more complex when characteristics such as tip shape and contact forces are taken into account. STM is generally applicable only to conducting samples while AFM is applied to both conductors and insulators. With regard to versatility, AFM is more capable. AFM can be operated in air, fluid, vacuum, and particular gaseous environments. 2), AFM versus SEM: Compared with Scanning Electron Microscope (SEM), AFM provides extraordinary topographic contrast, direct height measurements and unobscured views of surface features. AFM also offers atomic resolutions while SEM generally achieves only nanometer resolution. 3), AFM versus TEM: Compared with Transmission Electron Microscope (TEM), three-dimensional AFM 
images are obtained without expensive sample preparation and yield far more complete information than the two-dimensional profiles available from cross-sectioned samples in TEM. AFM generally is less expensive than TEM. Furthermore, both SEM and TEM are limited in vacuum conditions. 4), AFM versus optical microscope: Compared with optical interferometer, AFM provides unambiguous measurement of step heights, independent of reflectivity differences between materials. The unique AFM phase image allows a direct analysis with topography image simultaneously, realizing a comparison between shape and material property of surface structures. In terms of the concept of resolution, AFM is different from the other radiation-based microscopy techniques because AFM is a three-dimensional imaging technique. The ability to distinguish two separate points on an image is the standard by which lateral resolution is usually defined. There is clearly an important distinction between images resolved by wave optics and scanning cantilever techniques. The former is limited by diffraction, while the latter is limited primarily by cantilever and sample geometry.

\subsection{Tapping AFM in Liquids}

One of the advantages tapping mode AFM holds is its ability to image non-conducting and fragile surfaces. AFM measurement was quickly extended to liquid systems, since there are plenty of relevant molecules, materials or interactions where imaging in liquids is preferred or required. Unwanted forces between tip and surface may be neutralized by immersing tip and sample in liquids. Besides the elimination of capillary forces and the reduction of van der Waals forces, a fluid environment reduces or prevents tip and sample contaminations. Operation in liquids also makes it possible to study phenomena associated with liquid-gas interface such as surface bubbles, and biological interactions or reactions at real-time in situ, e.g., cell biologists with AFM have studied the dynamic behavior of living and fixed cells such as red blood cells, bacteria, platelets, renal epithelium cells, and so on.

The first tapping mode AFM operating in liquids was introduced in 1994 [6,7]. Since then, a large number of systems and methods have been studied. Tapping mode operation in fluid has the same advantages as in the air or vacuum. However, AFM experiments in liquids are difficult to perform and, in some cases, to understand [3]. The dynamics of the can- 
tilever motion in liquids is far more complex than in air. Imaging in a fluid medium tends to decrease the resonant frequency of cantilevers (typically 3 - 4 times less as compared to in air). In liquids, there are other kinds of acoustic vibrations, merging of some of cantilever vibration modes is also favored. The hydrodynamic damping between the liquid and the AFM cantilever also produces a substantial decrease of the cantilever quality factor (very effectively, e.g. 20 times less compared to the air in some cases). In addition, the liquid film confined between tip and sample surface may induce layering effects on the interactions. As a result, larger amount of peaks are present in the oscillation amplitude curve causing a complex at choosing driving frequency $[8,9]$. Nevertheless, when an appropriate frequency is selected (usually in the range of $5-40 \mathrm{kHz}$ ), the amplitude of the cantilever will decrease when the tip begins to tap the sample, similar to tapping mode operation in air. Alternatively, the very soft cantilevers can be used to get the good results in fluid. The spring constant is typically $0.1 \mathrm{~N} / \mathrm{m}$ compared to the tapping mode in air where the cantilever may be in the range of $1-100 \mathrm{~N} / \mathrm{m}$.

A comprehensive view of AFM tapping mode operation in liquids is still of lack, so far. The one-dimensional harmonic oscillator model has been introduced by Chen et al. to simulate cantilever motion in liquids. The model accounts for the changes in the fundamental frequency of the cantilever due to the added inertial mass of the liquid around the cantilever. It has been suggested that surface sensitivity can be achieved with driving frequencies above the resonance [10]. Thereafter, a hydrodynamic loading component due to the motion of the liquid around the cantilever and an external driving force that excites the cantilever have also been considered in the description of cantilever motion in liquids [11,12]. With respect to the effect that cantilever-surface proximity has on the liquid, it has been suggested that a compression of liquid expected near the surface gives rise to an interfacial stiffness (experimentally measured about $1 \mathrm{~N} / \mathrm{m}$ ). This interfacial stiffness is a dominant factor in the amplitude reduction and in the image contrast [13]. Also, an external signal proportional to the instantaneous deflection of the cantilever shifted by $90^{\circ}$ is introduced to enhance the quality factor of the cantilever, in order to minimize the effects of the broadening of the first resonance peak in liquids $[14,15]$.

Applications of AFM in liquids are chiefly with biosciences, by using phase imaging technique biologists can distinguish the different components of the cell membranes in solutions. Also, little sample preparation 
is required for liquid imaging with the AFM. In most cases it is as simple as spotting a few microliters of solution on a solid surface (e.g., mica or glass), of course after having avoided or removed the contaminations that cover surface features. Some of the most interesting force measurements have also been performed with surfaces under liquids where the environment can be quickly changed to adjust the concentration of various chemical components. In liquids, electrostatic forces between dissolved ions and other charged groups play an important role in determining the forces sensed by an AFM cantilever. The liquid environment has become an important stage for fundamental force measurements because researchers can control many of the details of the tip-surface interaction by adjusting properties of the liquids. In terms of surface nanobubbles, we see no other techniques that are able to allow experimentalists to study nanobubbles in such a reliable way as AFM is. Therefore, tapping mode AFM is the primary experimental method for the surface nanobubble issues. 


\section{References}

[1] Binnig, G.; Quate, C. F.; Gerber, Ch. Phys. Rev. Lett. 1986, 56, 930.

[2] Martin, Y.; Williams, C. C.; Wickramasinghe, H. K. J. Appl. Phys. 1987, $61,4723$.

[3] Garcia, R.; Pérez, R. Surf. Sci. Rep. 2002, 47, 197.

[4] Zhong, Q.; Imniss, D.; Kjoller, K.; Elings, V. B. Surf. Sci. 1993, 290, L688.

[5] edited by Morita, S.; Wiesendanger, R.; Meyer, E. Noncontact Atomic Force Microscopy Springer-Verlag Berlin Heidelberg 2002.

[6] Hansma, P. K.; Cleveland, J. P.; and et al. Appl. Phys. Lett. 1994, 64, 1738.

[7] Putmam, C. A.; van der Werf, K. O.; de Grooth, B. G.; van Hulst, N. F; Greve, J. Appl. Phys. Lett. 1994, 64, 2454.

[8] Schäffer, T. E.; Cleveland, J. P.; Ohnesorge, F. Walters, D. A.; Hansma, P.K. J. Appl. Phys. 1996, 80, 3622.

[9] O'Shea, S. J.; Lantz, M. A.; Tokumoto, H. Langmuir 1999, 15, 922.

[10] Chen, G. Y.; Warmack, R. J.; Huang, A.; Thundat, T. J. Appl. Phys. 1995, 78, 1465.

[11] Chon, J. W. M.; Mulvaney, P.; Sader, J. E. J. Appl. Phys. 2000, 87, 3978.

[12] Scherer, M. P.; Frank, G.; Gummer, A W. J. Appl. Phys. 2000, 88, 2912.

[13] Lantz, M.; Liu, Y. Z.; Cui, X.D. Tokumoto, H. Lindsay, S. M. Surf. Interf. Anal. 1999, 27, 354.

[14] Tamayo, J.; Humphris, A. D. L.; Owen, R. J.; Miles, M. J. Biophys. J. 2001, 81, 526.

[15] Anczykowski, B.; Cleveland, J. P.; Krüger, D.; Elings, V.; Fuchs, H. Appl. Phys. A 1998, 66, S885. 


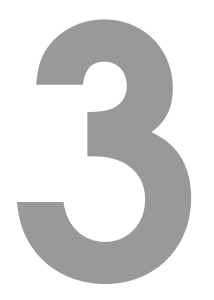

\section{Characterization of Nanobubbles on Hydrophobic Surfaces in Water}

The aim of this Chapter is to quantitatively characterize the appearance, stability, density, and shape of surface nanobubbles on hydrophobic surfaces under varying conditions such as temperature and temperature variation, gas type and concentration, surfactants, and surface treatment. The method we adopt is Atomic Force Microscopy (AFM) operated in the tapping mode. In particular, we show (i) that nanobubbles can slide along grooves under the influence of the AFM tip, (ii) that nanobubbles can spontaneously form by substrate heating, allowing for a comparison of the surface topology with and without the nanobubble, (iii) that a water temperature increase leads to a drastic increase in the nanobubble density, (iv) that pressurizing the water with $\mathrm{CO}_{2}$ also leads to a larger nanobubble density, but typically to smaller nanobubbles, (v) that alcoholcleaning of the surface is crucial for the formation of surface nanobubbles, (vi) that adding 2-butanol as surfactant leads to considerably smaller surface nanobubbles, and (vii) that flushing water over alcohol-covered surfaces strongly enhances the formation of surface nanobubbles.

Published as: Shangjiong Yang, Stephan Dammer, Nicolas Bremond, Harold Zandvliet, Stefan Kooij, and Detlef Lohse, Langmuir 2007, 23, 7072. 


\subsection{Introduction}

Previous studies have shown convincing experimental evidences for the existence and stability of nanobubbles [1-11], however, apart from those observations, fairly little about nanobubbles is known. For instance, how and why do nanobubbles form, and why are they apparently stable? What are the parameters that decisively impact the formation of nanobubbles? In order to help answering those puzzling questions, more studies on nanobubbles are necessary. The purpose of this Chapter is to elucidate these issues by performing AFM measurements of nanobubbles on hydrophobic silane-coated silicon wafer surfaces under varying conditions. Among the different substrates we tested, namely, hydrophobic or hydrophilic coated gold, bare silicon (with native oxidation layer), mica, and hydrophobilized silicon wafer with different coating agents, the hydrophobic silanated silicon wafer is chosen as the surface to form nanobubbles for further studies. The reason is following: first, as we know, hydrophobic surfaces favor nanobubble formation; second, the preparation method of the surface is mature, it provides us a physically and chemically homogeneous surface. The preparation method is addressed in the experimental section of this Chapter.

In this Chapter, the following experimental studies are performed. In terms of detecting liquid-gas interface, a direct comparison between nanobubbles and microcavity that traps air inside under water is established: we image nanobubbles next to the microcavity (an actual manufactured microbubble in the vicinity of nanobubbles). Nanobubble stability is examined: they remain stable on timescale of hours. We demonstrate the importance of surface topography to the nanobubbles. A link between surface roughness and nanobubbles is suggested: geometry of nanobubbles is in the similar geometric scale as the surface roughness. However, later studies show that nanobubbles can form at anywhere on the surface though with a preference to form at rougher locations, e.g., nanometer deep grooves on the surface. We also show the movement of a nanobubble along a surface groove, presumably under the influence of the scanning AFM tip. By increasing the temperature of substrate, nanobubbles are created in situ which allows to image exactly the same location with and without a nanobubble. This provides the topography information of the surface exactly underneath the nanobubbles. It is also found that alcohol prewashing process on the surface is influential on the nanobubble formation with regard to the nanobubble density and size. 
Furthermore, the effects of water temperature, gas concentration in water, surfactant added in water, and the so-called ethanol-water-exchange process are demonstrated. We see that increasing water temperature encourages the formation of nanobubbles, and nanobubbles do not disappear when the water cools down to room temperature. We also show that an increase of gas concentration of water facilitates nanobubble formation. This is supported by pressurizing the water with $\mathrm{CO}_{2}$ at different pressures which correspond to different gas concentrations in water. We see that nanobubble density is higher and nanobubble size is smaller at higher gas concentration, while under degassed water no nanobubbles are formed. The effect of surfactant is discussed with adding butanol drops into the water, which causes a decrease of radius of curvature of nanobubbles. Another method to efficiently enhance nanobubble formation is the ethanol-water-exchange process, i.e. the surface is initially covered by ethanol which is subsequently replaced by water. Finally, instead of water we used sodium chloride solution, and we see that nanobubbles are formed with similar size and density (compared to the water case) in the solution.

\subsection{Experimental Section}

In our experiments pure water is chiefly used as liquid, prepared by a Milli-Q Synthesis A10 system (Millipore SAS, France). Alcohols, i.e., ethanol, methanol, 2-propanol, and butanol of GR ( $>99.8 \%$, Merck KGaA, Germany) are used. $\mathrm{Si}(100)$ wafer pieces $(15 \mathrm{~mm} \times 15 \mathrm{~mm})$ are cleaned (Standard Wafer Clean, SWC) in clean-room conditions before they are coated by a hydrophobic monolayer (see below section). During the experiments, a syringe made of glass and metal (Poulten Graf, Germany) is used to add liquid drops onto the substrate. The syringe is rinsed with ethanol and pure water before use.

\subsubsection{Substrate preparation}

The solid substrate used in our experiments - a piece of a silicon wafer coated by a self-assembled hydrophobic monolayer - is prepared as follows. The wafer piece is boiled in a 3:1 mixture of concentrated sulfuric acid and hydrogen peroxide for 15 minutes. Then it is rinsed with pure 




Figure 3.1: AFM tapping mode topography image of the silane-coated silicon wafer surface (dry). The root mean square (RMS) value is $0.30 \mathrm{~nm}$ over the area shown in the image. The thickness of the silane monolayer is $1.0 \mathrm{~nm}$, suggested by the ellipsometry measurement.

water and ethanol (each for 1 minute) before it is left in an oven for 15 minutes at $110^{\circ} \mathrm{C}$. After this, the wafer piece is hydrophobilized by Chemical Vapor Deposition (CVD): it is left for 4 hours in a dry glass-chamber together with a drop of $1 \mathrm{H}, 1 \mathrm{H}, 2 \mathrm{H}, 2 \mathrm{H}$-Perfluorodecyldimethylchlorosilane, 90\% (16582, Lancaster Synthesis, England) at 10 mbar. Hereafter, the coated substrate is cleaned in an acetone ultrasonic bath for 1 minute. Ellipsometry measurements suggest that the thickness of the monolayer is approximately $1 \mathrm{~nm}$. The root mean square (RMS) value of the substrate in a $2 \mu \mathrm{m} \times 2 \mu \mathrm{m}$ area obtained by AFM measurements is $0.30 \mathrm{~nm}$. The advancing contact angle is $\theta=105^{\circ}$. A typical AFM topography image (tapping mode) of the surface is shown in Figure 3.1. After the preparation, the substrate is stored in a sample cell under clean-room conditions until it is used. Before each experiment, the substrate is cleaned in an ethanol ultrasonic bath for 15 minutes, and then it is blown dry for 1 minute with nitrogen gas. It is then mounted onto the AFM sample holder and inserted into the AFM. A drop of liquid is placed on the substrate where the AFM tip is measuring. Due to capillary forces the liquid drop stays there for a sufficiently long time (typically hours) to perform the measurements. 


\subsubsection{AFM imaging}

AFM measurements are done with a PicoSPM (Molecular Imaging, AZ, USA) in tapping mode. Excitation of the tip vibration is done acoustically, using a small piezo element in the tip-holder. A hydrophilic $\mathrm{Si}_{3} \mathrm{~N}_{4}$ ultrasharp AFM tip (NSC18/Al BS, MikroMasch, Spain) is used, with radius of curvature less than $10 \mathrm{~nm}$ (two orders of magnitude smaller than the radius of curvature of the nanobubbles to be observed), height about $22 \mu \mathrm{m}$, and full tip cone angle of $30^{\circ}$. For scanning in wet conditions the scanning speed is $4 \mu \mathrm{m} / \mathrm{s}$, the tapping mode free amplitude as applied to the cantilever is $400 \mathrm{mV}$, the set-point amplitude is $200 \mathrm{mV}$, and the frequency of the cantilever and the spring constant are approximately $20 \mathrm{kHz}$ and $0.9 \mathrm{~N} / \mathrm{m}$, respectively. The cantilever is cleaned by immersion in ethanol and pure water before use. All experiments are carried out in a general lab environment with a temperature between $20^{\circ} \mathrm{C}-23^{\circ} \mathrm{C}$.

\subsection{Results and Discussions}

\subsubsection{Nanobubbles next to a microbubble}

The response of the AFM cantilever over liquid-gas interface is studied with an artificial microbubble: a microcavity, etched in silicon wafer and then coated with silane monolayer making the surface hydrophobic, is used as the gas trap (Figure 3.2(a), SEM image of such a microcavity). The presence of gas inside is confirmed since a microscopic bubble emerges from the microcavity as soon as the liquid pressure is reduced [12]. The AFM phase image shown in Figure 3.2(b) exhibits numerous surface nanobubbles presenting a phase shift which is close to the shift over the liquidgas interface stretched on the microcavity. This phase signature is expected for softer objects (bubbles) than the solid surface. Phase analysis tells that the phase shift is $36^{\circ}$ on the microcavity and $23^{\circ}$ on the nanobubbles, with respect to the solid surface background. The reason for this degree difference between microcavity and nanobubbles is that on the deep microcavity the AFM cantilever responds to the pure air, however, on the thin nanobubbles the cantilever responds not only to the air but also partly to the solid surface which causes a weaker phase shift. 

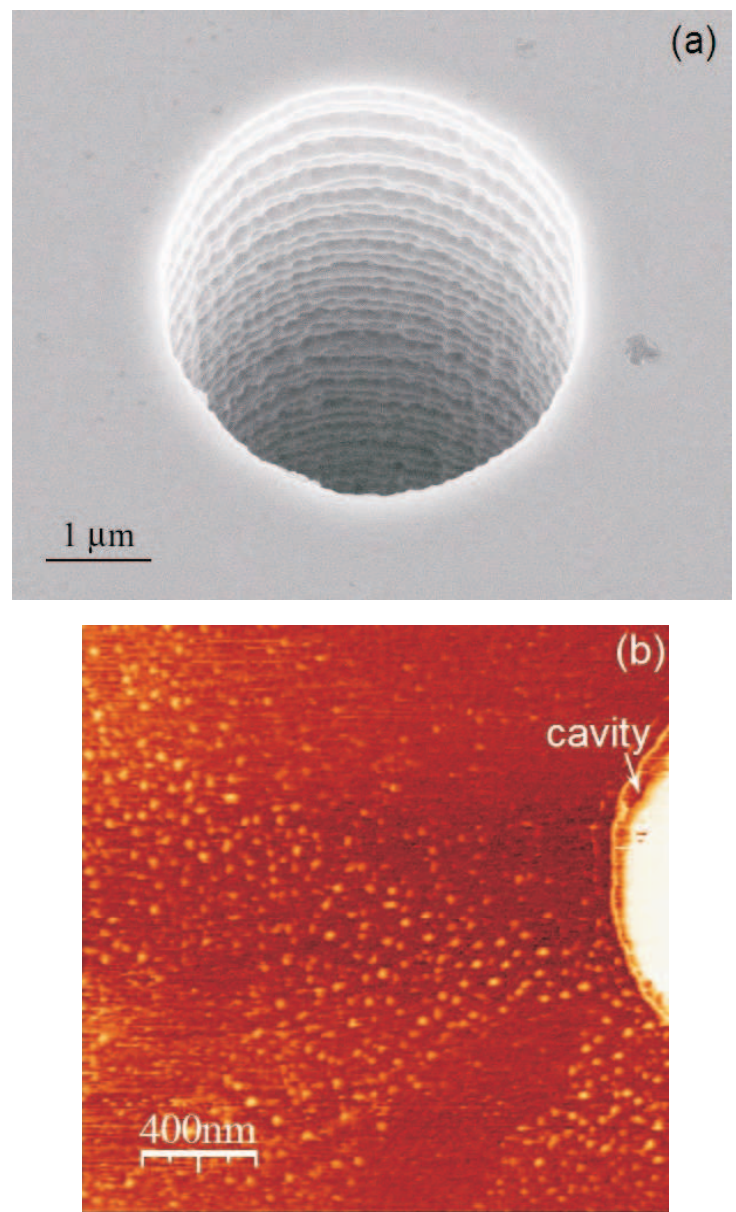

Figure 3.2: The response of the AFM cantilever over liquid-gas interface is studied with an artificial microbubble, i.e., a microcavity etched in silicon and then coated with a silane making the surface hydrophobic - it traps gas inside. Panel (a) shows the SEM image of the microcavity. Panel (b) shows the phase image of numerous nanobubbles that present a phase shift, sitting next to a microcavity. The phase shift of nanobubbles is close to that of the microcavity which is a rather strong shift due to the gas trapped inside. This phase signature is expected for softer objects (bubbles) than the solid surface. Phase analysis shows that the phase shift is $36^{\circ}$ on the cavity and $23^{\circ}$ on the nanobubbles, with respect to the surface background. This degree difference is due to the fact that on the deep microcavity the AFM cantilever responds to the pure air, however, on the thin nanobubbles the cantilever responds not only to the air but also to the solid surface which causes a weaker phase shift than the microcavity. 


\subsubsection{Nanobubble stability}

Previous studies have shown the surprisingly long lifetime of nanobubbles [1, $2,6]$. Here we tested the stability of nanobubbles on our silane surface. Nanobubbles were imaged by tapping mode AFM respectively at initial time and two hours later, with no other performances in between. It is shown that the nanobubbles remain very stable. An example is shown in Figure 3.3 (AFM topography and phase images): the lateral distance of the nanobubble is $150 \mathrm{~nm}$ in $\mathrm{x}$ direction and $240 \mathrm{~nm}$ in y direction, the height is $10 \mathrm{~nm}$, and the phase shift is $14.6^{\circ}$, initially. Two hours later, the size and phase of nanobubble remain stable: the lateral distance of the nanobubble is $140 \mathrm{~nm}$ in $\mathrm{x}$ direction and $225 \mathrm{~nm}$ in y direction, the height is 9.6 $\mathrm{nm}$, and the phase shift is $12.5^{\circ}$, as indicated in the figure. We presume that nanobubbles can be stable for much longer time. Interestingly, we see that the size of nanobubbles is similar to the surface roughness, i.e., a 2-5 nm local height deviation on an 100-500 $\mathrm{nm}$ lateral distance. Does the surface roughness stabilize nanobubbles? Later studies will give an answer to this question.

\subsubsection{Nanobubble 'sliding' along a groove}

AFM images of the surface in wet conditions (after placing drops of water on the substrate) at different time are shown in Figure 3.4. One can recognize a nanobubble located in the vicinity of a groove of depth $1 \mathrm{~nm}-2 \mathrm{~nm}$ which is presumably created by the wafer polishing process. The diameter of the nanobubble is about $25 \mathrm{~nm}$ and its height is approximately $3 \mathrm{~nm}$. The white star marks the same location in all images of Figure 3.4. Comparing these images, which show the nanobubble approximately in intervals of 3 minutes, one observes that the nanobubble is moving downward along the groove. We suggest that this directed motion is caused by the combined influence of the AFM tip, which drives the nanobubble, and the surface topography, i.e., the nanogroove. Surprisingly, there is no correlation between the slow and fast scan directions and the direction of movement of the nanobubble. While it has been reported previously that AFM tip can be used to move or merge nanobubbles (see for instance [4]), such an interplay between AFM tip and surface topography is a novel feature which potentially offers new means of manipulating nanobubbles. Note that the bubble is not located in the groove but beside it, for which we have no explanation. 

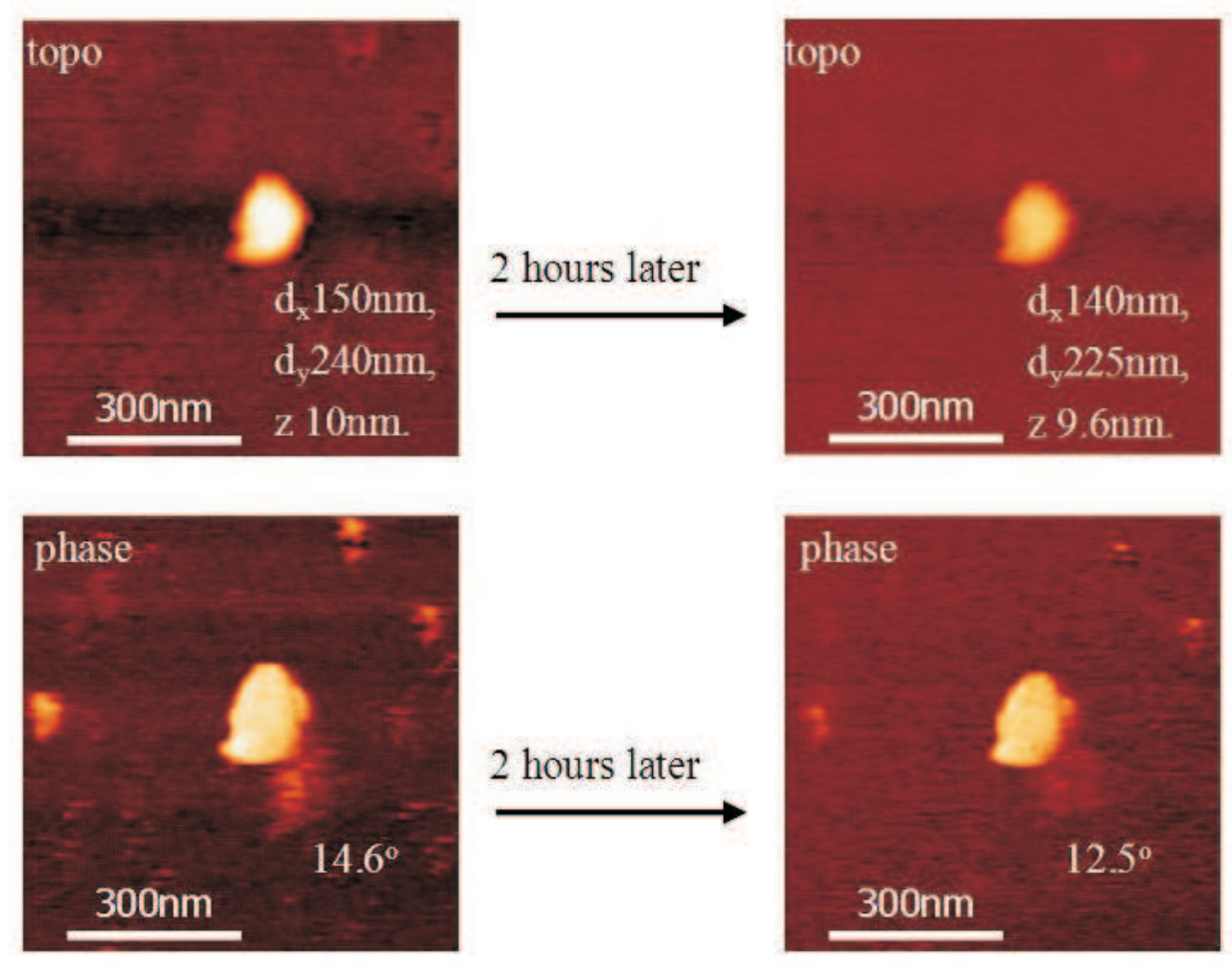

Figure 3.3: AFM topography and phase images of a nanobubble at initial time are shown on the left side. Two hours later the nanobubble is imaged again, its topography and phase images are shown on the right side. The lateral distance of the nanobubble initially is $150 \mathrm{~nm}$ in $\mathrm{x}$ direction and $240 \mathrm{~nm}$ in y direction, the height is $10 \mathrm{~nm}$, and the phase is $14.6^{\circ}$. The size and phase of nanobubble remain stable after two hours. The lateral distance of the nanobubble then is $140 \mathrm{~nm}$ in $\mathrm{x}$ direction and $225 \mathrm{~nm}$ in $\mathrm{y}$ direction, the height is $9.6 \mathrm{~nm}$, and the phase shift is $12.5^{\circ}$. Presumably, nanobubbles can even stay stable for a longer time than two hours. 

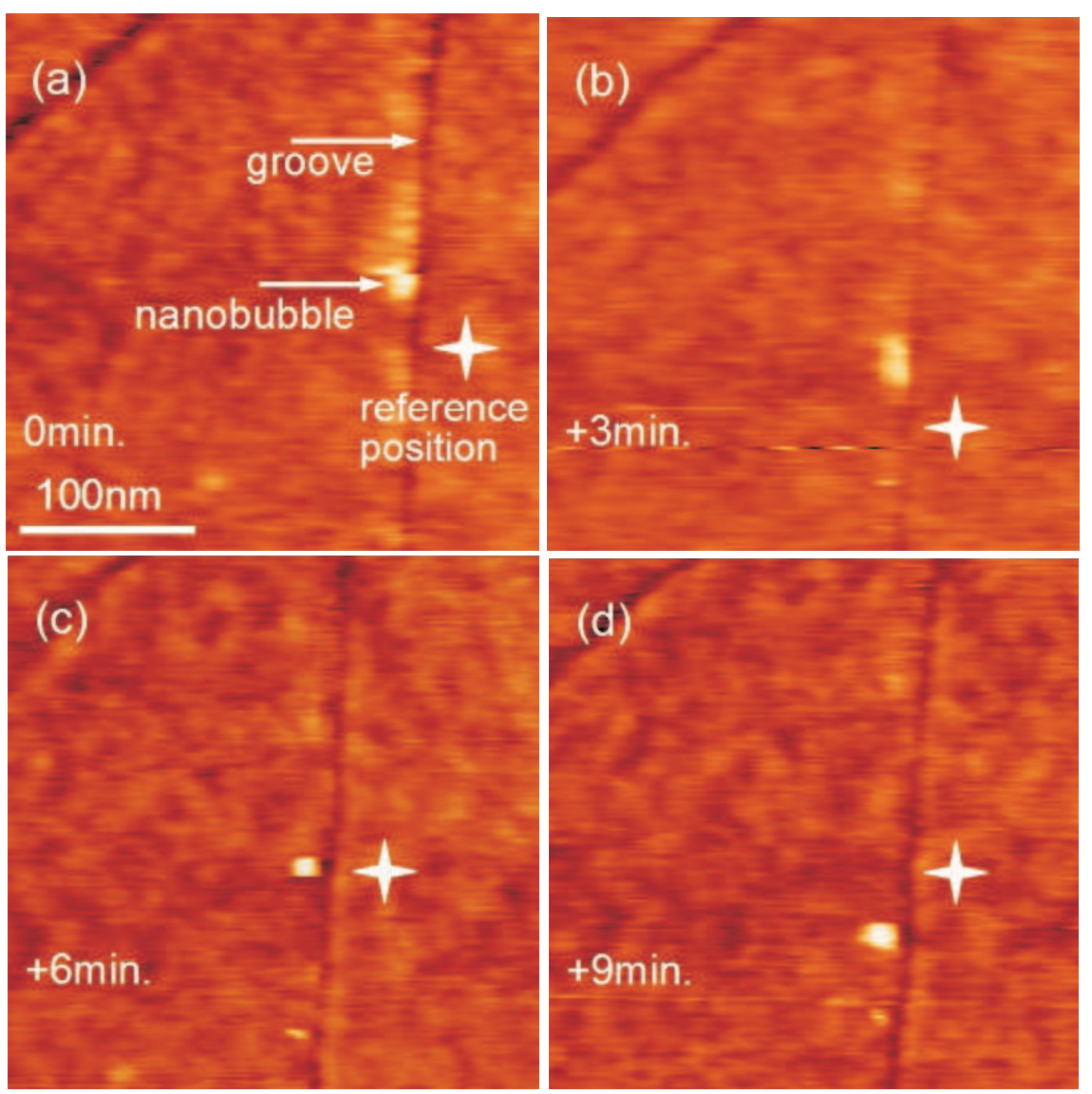

Figure 3.4: AFM topography images (tapping mode, height range: $4.4 \mathrm{~nm}$ ) of a nanobubble ( $25 \mathrm{~nm}$ wide, $3 \mathrm{~nm}$ high) next to a groove $(1 \mathrm{~nm}-2 \mathrm{~nm}$ deep) on the substrate. The images are recorded continuously one after another, while recording one image takes 3 minutes. Successive horizontal line scans (from left to right and right to left) are done, with scanning direction from the bottom of the images to the top. Image (a) is recorded immediately after a drop of water is placed on the substrate, and images (b), (c), and (d) record the position of the nanobubble approximately after 3, 6, and 9 minutes, respectively. To give a reference position, the white star marks the same location in each image. As the scanning direction is both left to right and right to left, presumably the surface topography is the origin for the surface nanobubble to be on the left hand side of the groove. Remarkably, the nanobubble moves downwards along the groove. This is opposite to the bottom-top scanning direction. We do not understand the details of interaction between tip, bubble, and substrate topography. 


\subsubsection{In situ creation of nanobubbles by substrate heating}

Previously it has been reported that an increase of the liquid temperature favors the formation of nanobubbles [8]. Here we adopt this method to create nanobubbles in situ. We heat the substrate by a heating stage (a piece of copper mounted underneath the substrate and heated by an electric current). Figure 3.5 shows AFM images of the surface (under water) at different substrate temperature. It is clearly presented that an increase of the substrate temperature leads to an increase in the number of nanobubbles, in particular between $25^{\circ} \mathrm{C}$ and $30^{\circ} \mathrm{C}$. Furthermore, nanobubbles also disappear and merge. In agreement with previous studies, nanobubbles can form on the flat surface (position 1 in Figure 3.5), however, the majority of nanobubbles formed by heating of the substrate is located in the vicinity of grooves. This observation is similar to [7] where nanobubbles on a HOPG surface were preferentially found near atomic steps. Note that as in Figure 3.4 the nanobubbles are rather located beside the grooves than in the grooves. Summarizing this experiment it indicates that the surface topography can have a considerable influence on the formation of nanobubbles. However, surface structures such as grooves are not a necessary condition for the formation of nanobubbles.

Examples of nanobubbles that grow and merge are given in Figure 3.6 (AFM tapping mode topography images). A nanobubble of height 10.1 $\mathrm{nm}$ and width $49 \mathrm{~nm}$ is shown in image (a). The nanobubble expands after the substrate temperature has been increased of $5^{\circ} \mathrm{C}$. Image (b) shows that the nanobubble develops till with a size of height $10.3 \mathrm{~nm}$ and width $58 \mathrm{~nm}$. The volumes of the nanobubble in the two cases are estimated as follows: $0.7 \times 10^{4} \mathrm{~nm}^{3}$ in (a) while $1.4 \times 10^{4} \mathrm{~nm}^{3}$ in (b). In Figure 3.6(c) two nanobubbles sitting next to each other at a lower substrate temperature are presented. The two nanobubbles merged into one after the substrate temperature raised $5^{\circ} \mathrm{C}$, as shown in image (d). The estimated width, height, and volume for the nanobubbles in image (c) is $112 \mathrm{~nm}, 10.3 \mathrm{~nm}$, and $3.6 \times 10^{4} \mathrm{~nm}^{3}$ for the bigger one, and $63 \mathrm{~nm}, 5.4 \mathrm{~nm}$, and $0.7 \times 10^{4} \mathrm{~nm}^{3}$ for the smaller one. The size of the merged one is $115 \mathrm{~nm}$ in width, $10.1 \mathrm{~nm}$ in height, and $3.8 \times 10^{4} \mathrm{~nm}^{3}$ in volume as shown in (d). However, note that the volume of the merged bubble is not exactly the sum of the former two nanobubbles.

How is the height profile of a nanobubble compared to the topography of the underlying substrate? In previous studies this was merely investigated in an averaged sense: comparing the typical surface topography 


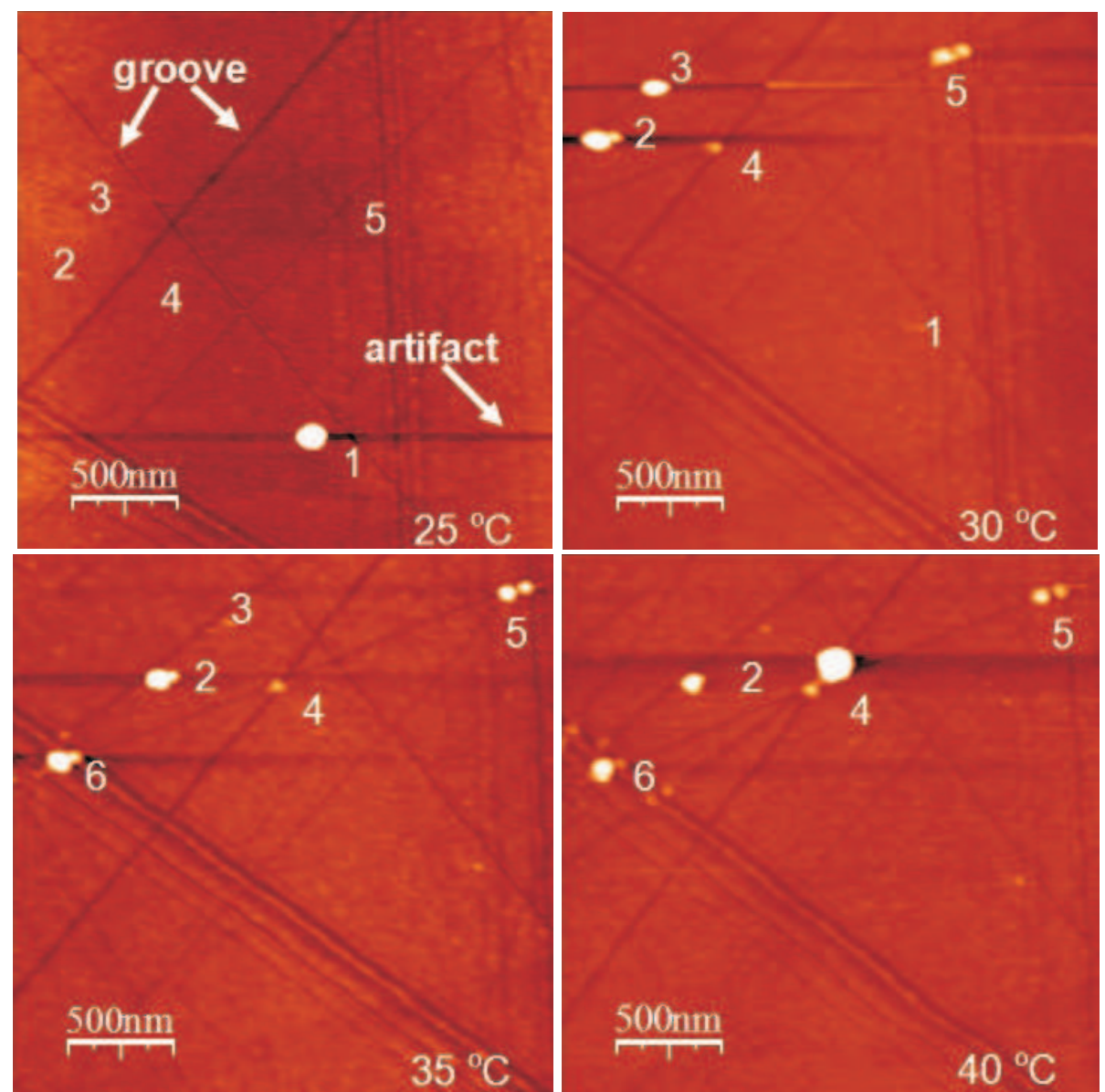

Figure 3.5: AFM topography images (tapping mode, height range: $26.3 \mathrm{~nm}$ ) at different substrate temperatures. The temperature is increased in situ. The imaging area drifts slightly due to thermal drift. The grooves in the surface are approximately $1 \mathrm{~nm}-2 \mathrm{~nm}$ deep. Numbers mark locations of interest, i.e., locations where nanobubbles appear and disappear. Locations 2-6 are in the vicinity of grooves. Due to a varying contrast of the images the grooves are not equally visible in different images. Note that the dark horizontal stripes at the height of the nanobubbles are artifacts from the imaging. As the temperature is increased, more nanobubbles appear, though nanobubbles also disappear or merge. Nanobubbles may form on the flat surface, e.g., at position 1. Nevertheless, the majority of nanobubbles forms in the vicinity of grooves, i.e., positions 2-6. 

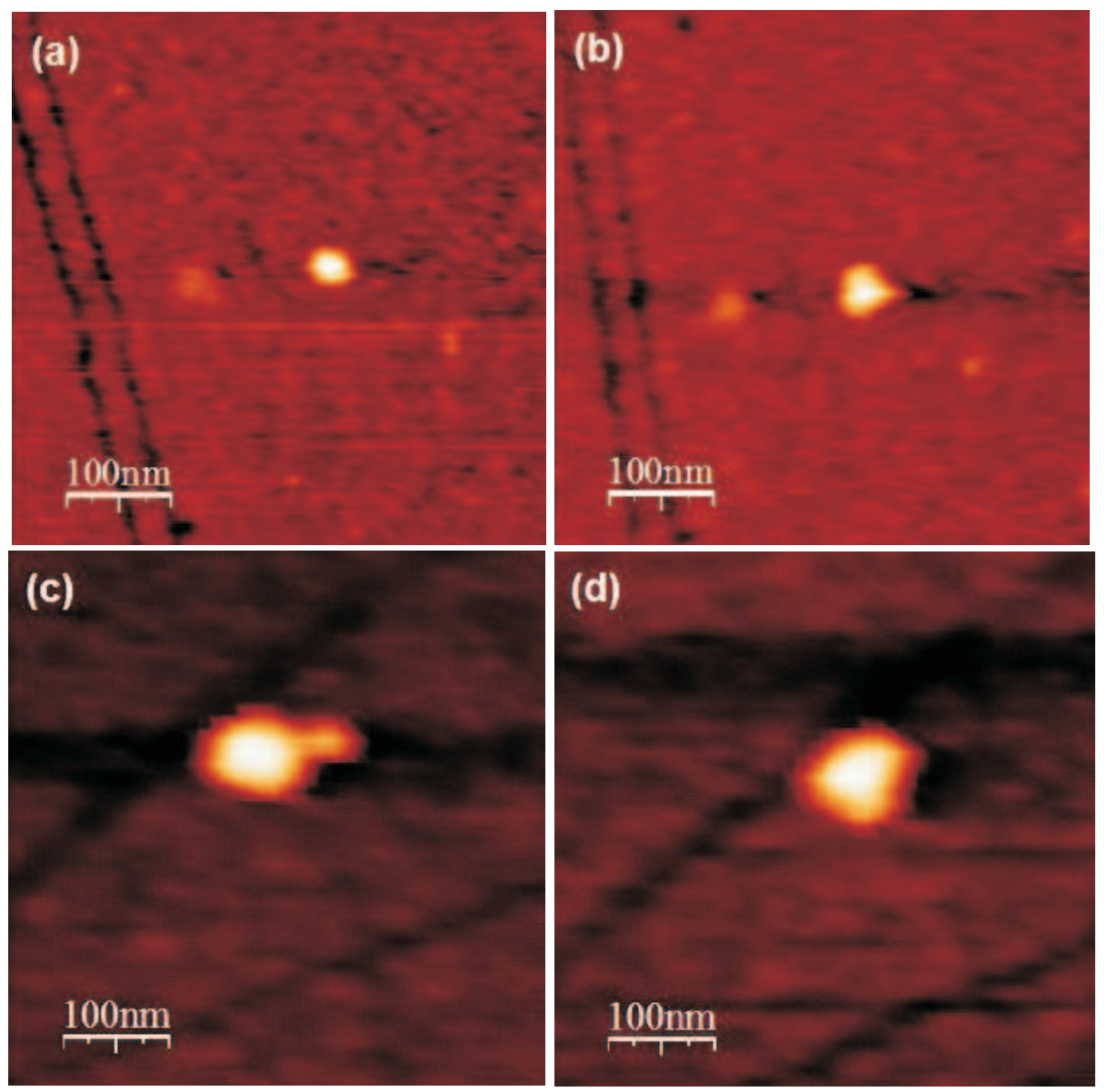

Figure 3.6: Examples of nanobubbles growing and merging (AFM tapping mode topography images). Image (a) shows a nanobubble of height 10.1 $\mathrm{nm}$ and width $49 \mathrm{~nm}$. After having increased the substrate temperature by $5^{\circ} \mathrm{C}$, the nanobubble grows till a size of height $10.3 \mathrm{~nm}$ and width 58 $\mathrm{nm}$, as shown in image (b). The volumes of the nanobubble in the two cases are estimated: $0.7 \times 10^{4} \mathrm{~nm}^{3}$ in (a) whereas $1.4 \times 10^{4} \mathrm{~nm}^{3}$ in (b). In panel (c) it shows two nanobubbles sitting next to each other at a lower substrate temperature. The two merged into one after the substrate temperature raised $5^{\circ} \mathrm{C}$. The estimated width, height, and volume for the two nanobubbles in image (c) are $112 \mathrm{~nm}, 10.3 \mathrm{~nm}$, and $3.6 \times 10^{4} \mathrm{~nm}^{3}$ for one, and $63 \mathrm{~nm}, 5.4 \mathrm{~nm}$, and $0.7 \times 10^{4} \mathrm{~nm}^{3}$ for the other. The size of the merged one in image (d) is $115 \mathrm{~nm}$ in width, $10.1 \mathrm{~nm}$ in height, and $3.8 \times 10^{4} \mathrm{~nm}^{3}$ in volume. 
(e.g., the RMS value) of the dry surface with the typical nanobubble profiles of the wet surfaces. This method does not yield information on the surface topography beneath a given nanobubble. Superior to this, the in situ creation of nanobubbles by heating of the substrate allows to scan exactly the same location on the surface in wet conditions with and without a nanobubble. By this, a direct comparison between the nanobubble profile and the underlying surface is possible. Figure 3.7 shows AFM images at a substrate temperature of $25^{\circ} \mathrm{C}$ and $30^{\circ} \mathrm{C}$.

For the lower temperature, in Figure 3.7(a), there is no nanobubble in the upper part of the image, hence, the surface topography is imaged. The increase of the temperature by $5^{\circ} \mathrm{C}$ leads to the formation of a nanobubble, which is imaged in Figure 3.7(b). Figure 3.7(c) compares the nanobubble profile with the topography of the underlying substrate. The good agreement in the topography between Figure 3.7(a) and Figure 3.7(b) away from the bubble evidences that the AFM is not considerably perturbed by the increase of the temperature or the nanobubble, hence, the two different measurements can be compared. Figure 3.7(c) explicitly shows that the height scale of the nanobubble is much larger than the scale associated with the roughness of the underlying substrate. Figure 3.7(d) illustrates that the nanobubble profile resembles a spherical cap with nanoscopic contact angle $\theta_{\mathrm{n}} \approx 164^{\circ}$ much larger than the macroscopic (advancing) contact angle $\theta=105^{\circ}$. The associated radius of curvature is $R \approx$ $217 \mathrm{~nm}$. In order to demonstrate that these results are reproducible, Figure 3.7(e,f,g,h) show analogous results to Figure 3.7(a,b,c,d) for a different location on the surface. Note that similar to the investigations mentioned above the nanobubble in Figure 3.7(f) is close to a groove. Nevertheless, the depth of the groove is considerably smaller than the height of the nanobubble.

\subsubsection{Increase of water temperature}

In contrast to the method described in the previous section, here we quickly (time scale $\approx 2$ minutes) heat up the water in a beaker under general lab conditions. When the water reaches the desired temperature it is immediately put onto the substrate by the syringe. Then, the AFM measurement follows. Figure 3.8 shows AFM images at different water temperature during immersion of the substrate, ranging in intervals of $5^{\circ} \mathrm{C}$ from $20^{\circ} \mathrm{C}$ (a) to $40^{\circ} \mathrm{C}(\mathrm{e})$. An increase of the water temperature leads to an increase 

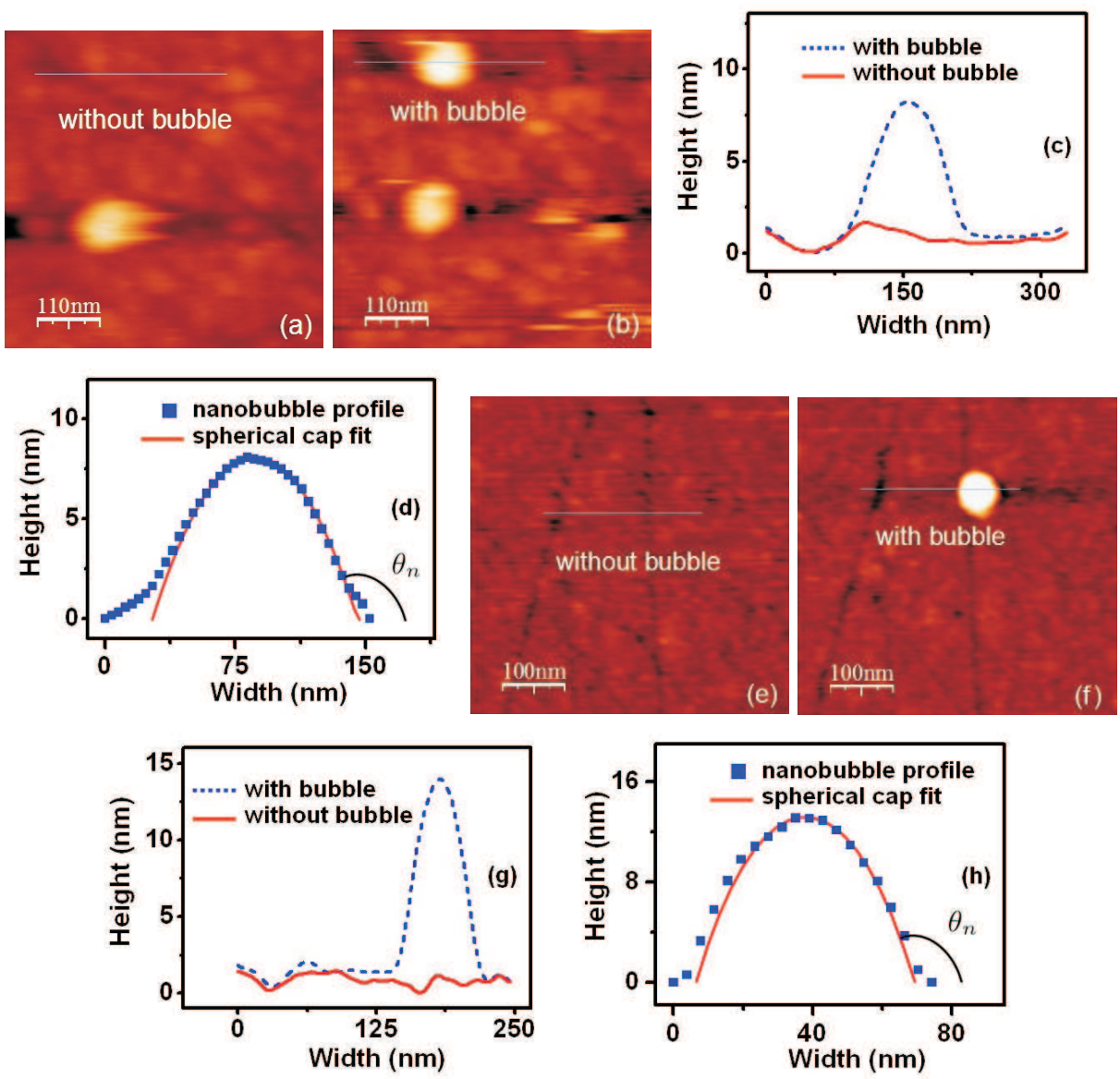

Figure 3.7: (a) and (b) AFM topography images (tapping mode, height range: $9.9 \mathrm{~nm}$ ) at $25^{\circ} \mathrm{C}$ and $30^{\circ} \mathrm{C}$ substrate temperatures, respectively. An increase of the substrate temperature leads to the formation of a nanobubble in (b). This makes it possible to image exactly the same location with and without a nanobubble. Plot (c) shows the cross-sectional profiles of the nanobubble and the underlying substrate, corresponding to scans along the lines depicted in images (a) and (b). Plot (d) shows the nanobubble profile in comparison to a spherical cap fit, from which the nanoscopic contact angle $\theta_{n}=164^{\circ}$ is obtained. Note the different scales on the width and height axes in (c) and (d). (e)-(h): analogous to (a)(d). The height range in (e) and (f): $15.2 \mathrm{~nm}$; nanoscopic contact angle $\theta_{\mathrm{n}}=137^{\circ}$ in (h). 


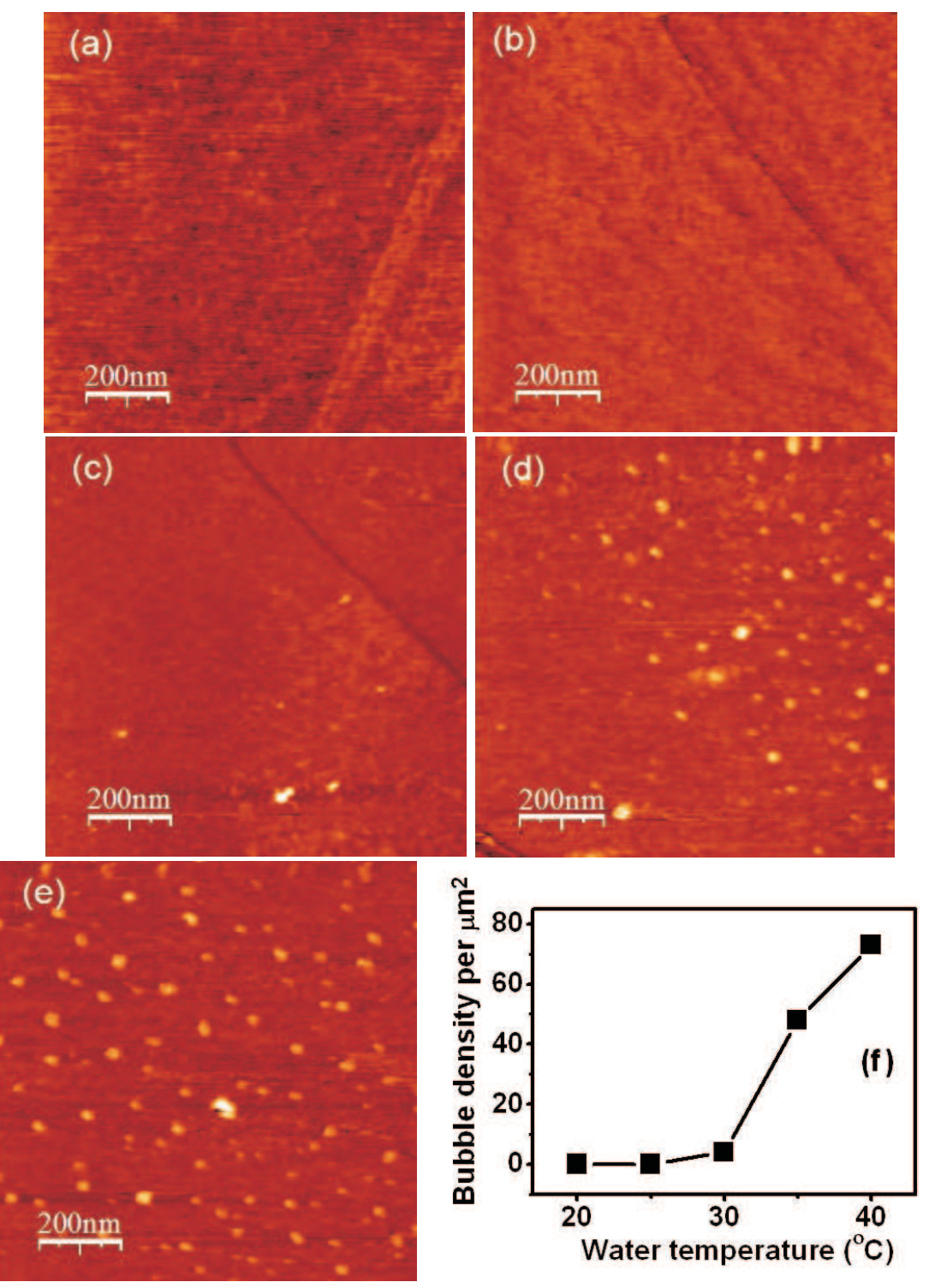

Figure 3.8: AFM topography images (tapping mode, height range: $11.1 \mathrm{~nm}$ ) at different water temperature during immersion of the substrate. The water temperature ranges in intervals of $5^{\circ} \mathrm{C}$ from $20^{\circ} \mathrm{C}$ (a) to $40^{\circ} \mathrm{C}(\mathrm{e})$. The dependence of the nanobubble density with water temperature is depicted in (f), showing a dramatic increase of the nanobubble density from $30^{\circ} \mathrm{C}$ to $35^{\circ} \mathrm{C}$. Presumably, the increase of the water temperature effectively leads to an oversaturation with gas of the water, facilitating nanobubble formation. Note that the nanobubbles do not disappear when the water cools down again to ambient conditions. 
of the nanobubble density, which is illustrated in Figure 3.8(f). In particular, there is a dramatic change in the nanobubble density from $30^{\circ} \mathrm{C}$ to $35^{\circ} \mathrm{C}$, an observation similar to [8]. Since the gas solubility decreases with increasing temperature and heat diffusion is orders of magnitude larger than gas diffusion we expect that the water is oversaturated with air during the immersion of the substrate and that this favors the formation of nanobubbles. Surprisingly, even when the water cools down again to ambient conditions, the nanobubbles do not disappear, indicating that the conditions during immersion are crucial for the formation of nanobubbles. Once they are formed they are remarkably stable. This interpretation is in accordance with [13] where it is demonstrated that nanobubbles, once they are formed, are stable against immense reduction of the liquid pressure.

\subsubsection{Pressurizing the water with $\mathrm{CO}_{2}$}

To further support that an increase of the gas saturation of the water enhances nanobubble formation, we pressurize the water with $\mathrm{CO}_{2}$ at different pressures. Therefore, first the water is partially degassed by keeping it for 1 hour under a reduced pressure of 30 mbar (as a test experiment, the degassed water is added on the surface, and we found that no nanobubbles are formed). Then the degassed water is put into a metal container, while $\mathrm{CO}_{2}$ gas at the desired pressure is pumped into the container as well. Thereafter, the container is sealed for 3 hours, leaving the water under a $\mathrm{CO}_{2}$ atmosphere. Subsequently the water is put onto the substrate, immediately followed by the AFM measurements at ambient lab conditions. We measure the $\mathrm{CO}_{2}$ concentration in the water by its $\mathrm{pH}$ $\mathrm{kH}$ values (The $\mathrm{kH}$ value is a measure of bicarbonate and carbonate ions that act as buffers to prevent the $\mathrm{pH}$ dropping. One degree $\mathrm{kH}$ is equivalent to $17.9 \mathrm{mg} / \mathrm{ltr} \mathrm{CaCO}_{3}$.). Figure 3.9(a,b,c) show AFM images for water pressurized with 1,2 , and 3 bar. Indeed, the density of nanobubbles increases with increasing $\mathrm{CO}_{2}$ pressure. Furthermore, the nanobubbles tend to cluster with increasing $\mathrm{CO}_{2}$ pressure. However, this might also be caused by the underlying substrate. To characterize the shape of the nanobubbles, in Figure 3.9(d) we plot the width over the height of the nanobubbles versus their height. There appears to be a trend that the nanobubbles are smaller for increasing pressure, which might also be related to the clustering of the nanobubbles. 

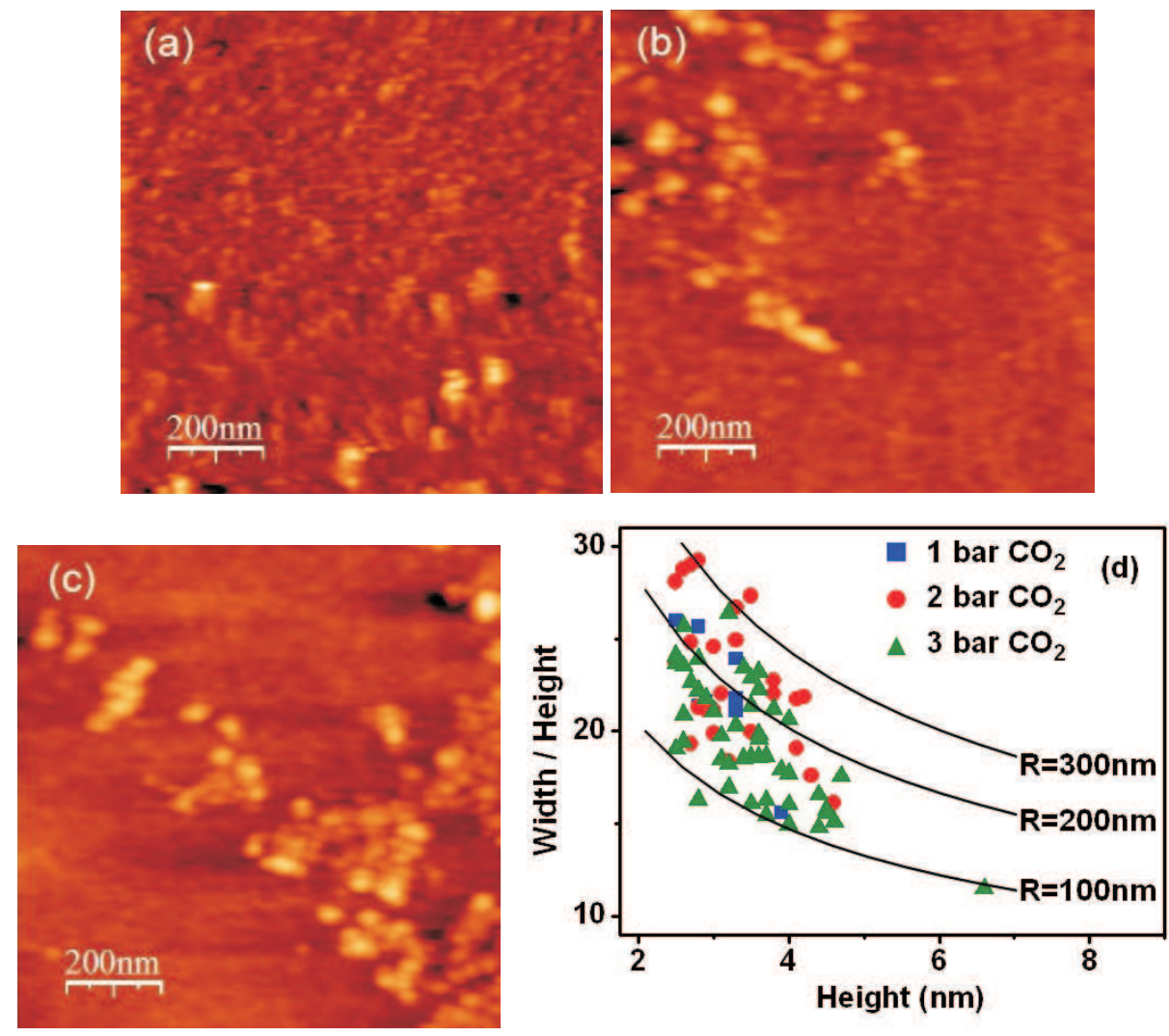

Figure 3.9: (a)-(c) AFM topography images (tapping mode, height range: $7.1 \mathrm{~nm}$ ) for water pressurized with $\mathrm{CO}_{2}$ at different pressures, leading to different $\mathrm{CO}_{2}$ concentrations. (a): 1 bar, $50.6 \mathrm{mg} / \mathrm{ltr}$; (b): 2 bar, 186.7 $\mathrm{mg} / \mathrm{ltr}$; (c): 3 bar, $379.4 \mathrm{mg} / \mathrm{ltr}$. The nanobubble density is 9,25 , and 46 nanobubbles per $\mu \mathrm{m}^{2}$ for (a), (b), and (c), respectively. Plot (d) shows the width over height of the nanobubbles versus the height. The solid line correspond to spherical caps with different radii of curvature $R$. 

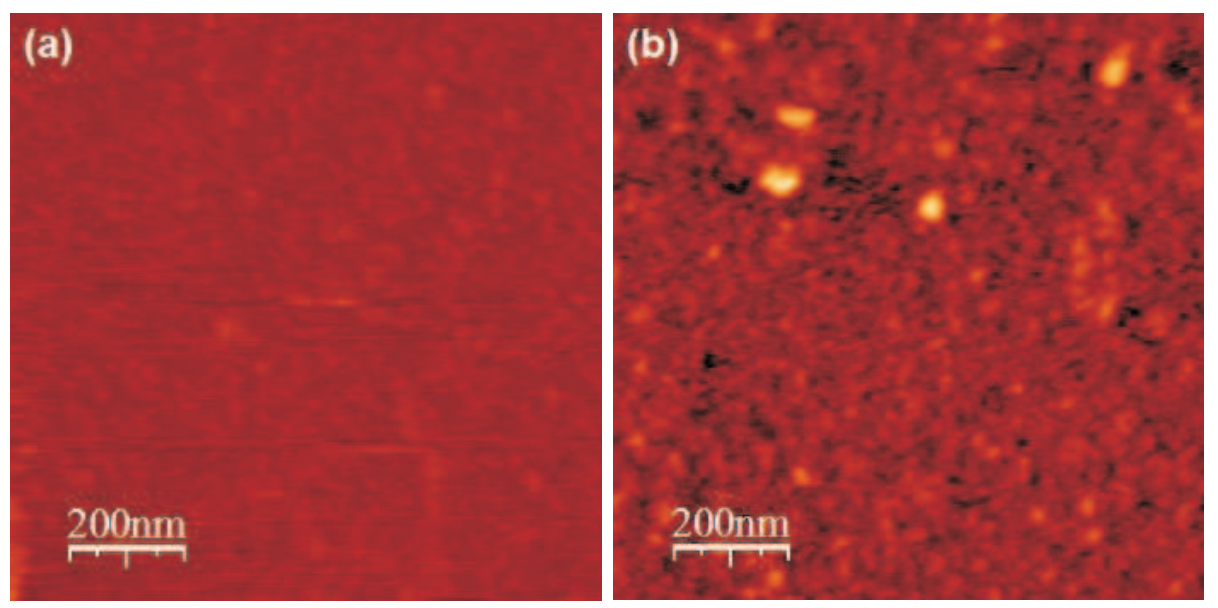

Figure 3.10: The alcohol prewashing process is crucial for the formation of nanobubbles on the silane sample. If the water is added on the surface without the alcohol treatment, nanobubbles are hardly found, as shown by the AFM topography image (height range $7.7 \mathrm{~nm}$ ) in panel (a). After having the alcohol prewashing process, nanobubbles form on the surface, as shown in panel (b).

\subsubsection{Influence of cleaning procedure}

As described above, the standard sample preparation includes an ultrasonic bath of the substrate in ethanol, after which it is blown dry by nitrogen gas. The prewashing process with ethanol is crucial [14]: without the ethanol treatment we hardly find any nanobubbles when the substrates are immersed in water. Figure 3.10 presents the AFM images of the surface (under water), (a) without the ethanol prewashing, (b) with the ethanol prewashing. As we can see, no nanobubbles are found in image (a), whereas some nanobubbles are formed in image (b). We do not have a proper explanation for the decisive role of the ethanol treatment. However, as far as we are concerned, a reason might be that ethanol with its low vapor pressure removes residual water from the substrate, which could have condensed on the surface from the ambient air. To further investigate the role of the surface preparation, instead of ethanol we use 2-propanol and methanol as cleaning agent of the ultrasonic bath. Figure 3.11 shows data obtained from AFM measurements after treating the substrate with different alcohols. 

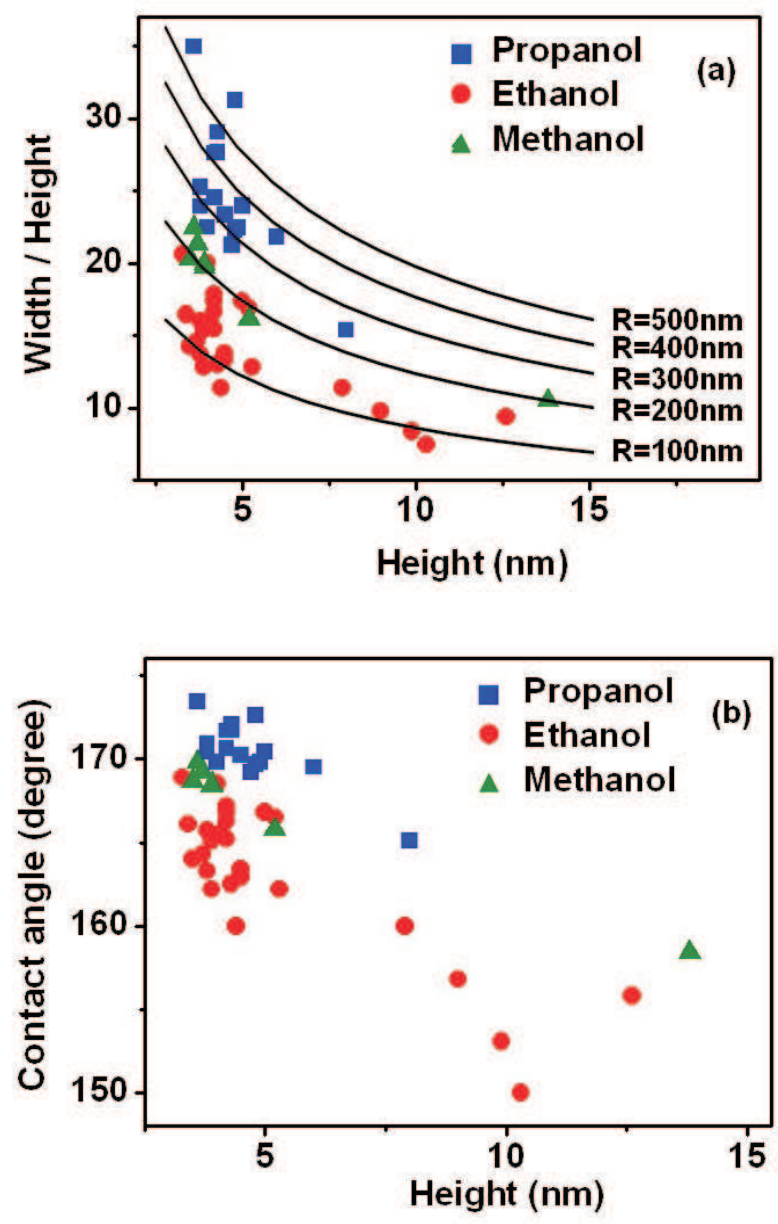

Figure 3.11: Width over height versus height (a), and nanoscopic contact angle (b) of nanobubbles, obtained by AFM measurements after cleaning the substrate with different alcohols. The density of nanobubbles in this experiments is 7,28 , and 17 per $5 \mu \mathrm{m}^{2}$ for methanol, ethanol, and 2-propanol, respectively. (b) is obtained from (a) by assuming a spherical cap bubble. Solid lines in (a) correspond to spherical caps with different radii of curvature R. One can observe a trend in the data, i.e., the radii of curvature and the contact angles decrease in the order 2-propanol, methanol, and ethanol. 
Both the width over height versus height plot, Figure 3.11(a), as well as the nanoscopic contact angle, Figure 3.11(b), show a trend towards decreasing width over height and decreasing contact angle in the order 2propanol, methanol, ethanol. Note that this order does not agree with the chain length of the alcohols, namely, 2-propanol, ethanol, methanol from long to short chain lengths. Definitely, the formation of nanobubbles as well as their morphology are sensitive to the substrate cleaning procedure.

\subsubsection{Adding a surfactant}

Surfactant is able to change the morphology of nanobubbles, a phenomenon which has been studied in [6]. We add to these results experiments in which we add a drop of 2-butanol to the drop of water on the substrate, after nanobubbles have already been formed and were imaged (without butanol). The volume ratio is 1(2-butanol):5(water). Figure 3.12(a,b) show nanobubbles in pure water, and after adding butanol to the water on the substrate, respectively. As shown in Figure 3.12(c), there is a slight decrease in the height of the nanobubbles, while the decrease in width is more pronounced. Overall, this clearly shows that the radius of curvature, as indicated by the solid lines in Figure 3.12(c), is reduced from $351 \mathrm{~nm}$ and $470 \mathrm{~nm}$ for the bubbles A1 and B1, respectively, to values of $122 \mathrm{~nm}$ and $294 \mathrm{~nm}$ for the two bubbles. Correspondingly, the nanoscopic contact angles decrease from $163^{\circ}$ and $168^{\circ}$ to $152^{\circ}$ and $166^{\circ}$, respectively.

\subsubsection{Ethanol-water exchange}

In the above experiments we followed the standard method of substrate preparation described above, and placed a drop of water on the substrate, which leads to the formation of nanobubbles. Is it possible to further enhance the nanobubble density by a different preparation of the substrate? It has been claimed $[6,8]$ that the formation of nanobubbles is stimulated when the substrate is first covered by ethanol which is then flushed away by water. This has been confirmed even for hydrophilic bare silicon oxide surfaces [9]. Here, we adopt this method to check if it also applies for our substrates. In addition, we also use 2-propanol instead of ethanol to be flushed away by water on the surface. Figure 3.13 shows the effect of such an alcohol-water exchange on the topography of the liquid-solid 

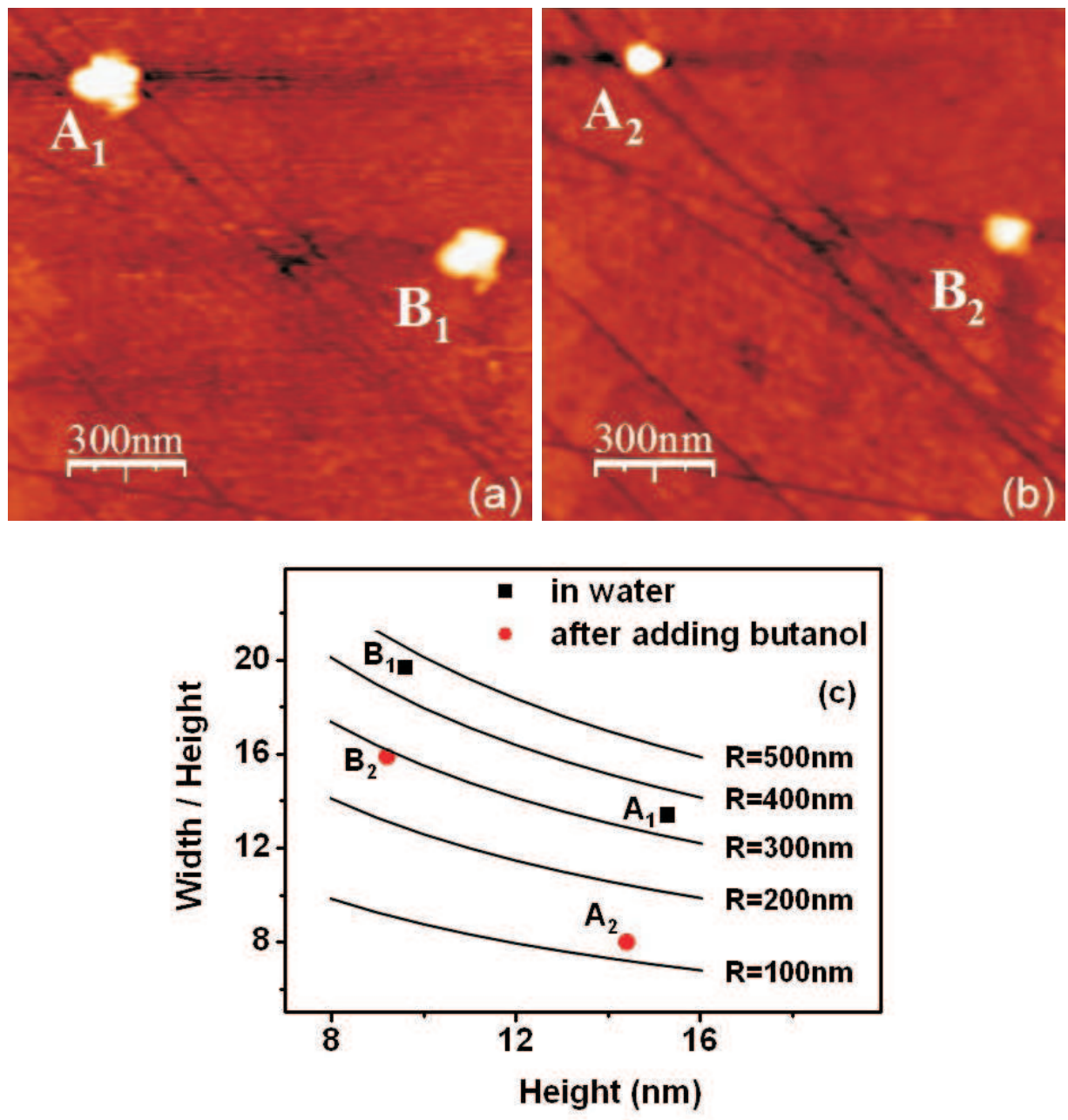

Figure 3.12: AFM topography images (tapping mode, height range: $16.5 \mathrm{~nm}$ ) before (a) and after (b) adding a drop of butanol to the water on the substrate (volume ratio of water to butanol is 5:1). Both nanobubbles show a decrease of width and height, though the decrease in height is less pronounced. Consequently, the radius of curvature decreases from $351 \mathrm{~nm}$ and $470 \mathrm{~nm}$ for the bubbles A1 and B1 to $122 \mathrm{~nm}$ and $294 \mathrm{~nm}$, respectively. The nanoscopic contact angles decrease from $163^{\circ}$ and $168^{\circ}$ to $152^{\circ}$ and $166^{\circ}$ respectively for bubbles A1 and B1. We note that it may be possible that micelles are formed. The critical micelles concentration for butanol is $\Gamma_{\mathrm{s}, \mathrm{crit}}=\left(0.25 \mathrm{~nm}^{2}\right)^{-1}[16]$. 


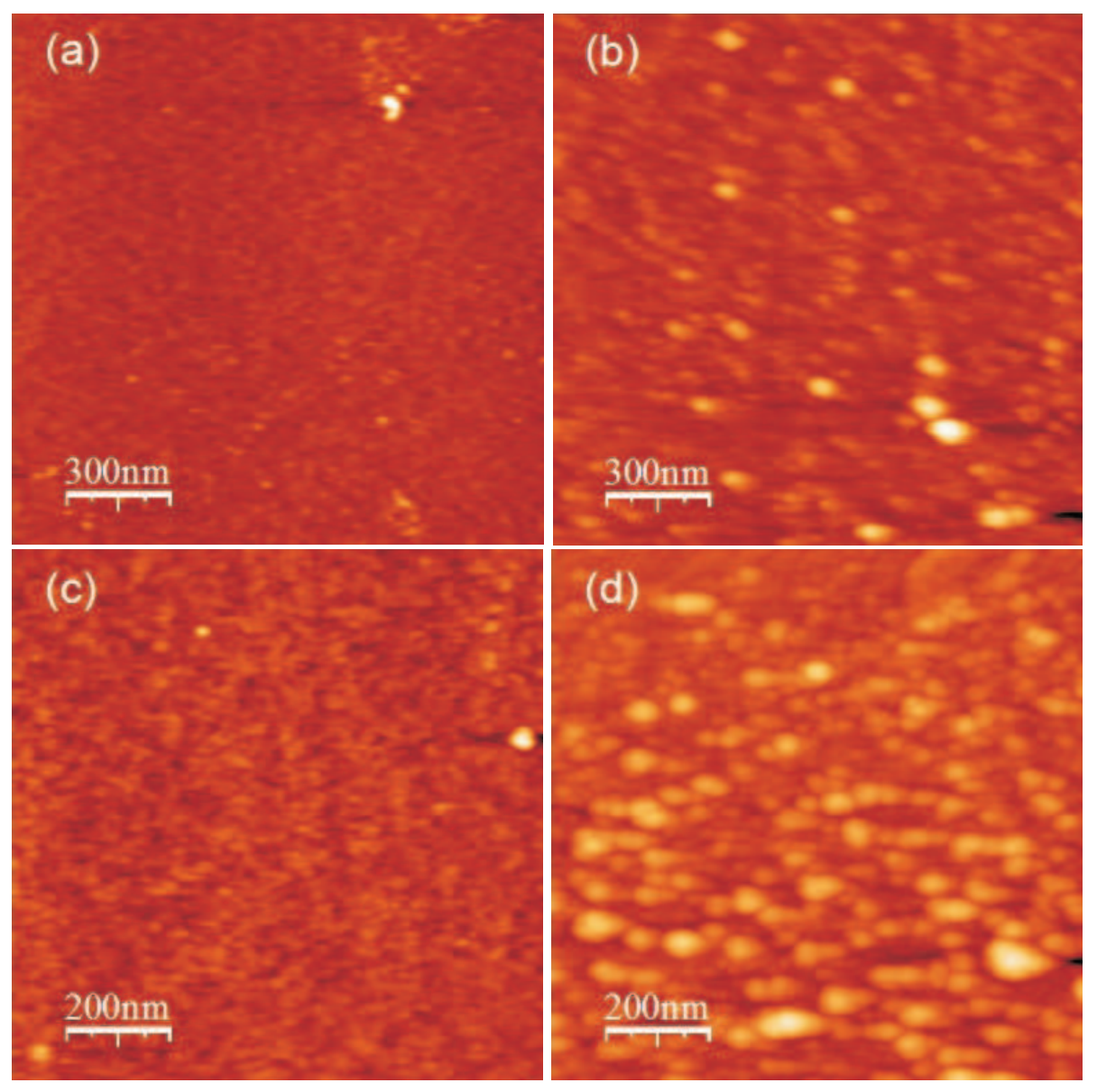

Figure 3.13: AFM topography images (tapping mode, height range: $7.5 \mathrm{~nm}$ (a) and (b), height range: $8.6 \mathrm{~nm}$ (c) and (d)) of the substrate immersed in (a) propanol and (c) ethanol. Only very few nanobubbles form in the alcohols. Images (b) and (d) show the substrate after propanol and ethanol, respectively, have been flushed away by water. The density of nanobubbles is dramatically increased after replacing the alcohols by water, as reported in $[6,8,9]$. 
interface. As one can see in the figure, AFM topography images of the substrate immersed in (a) propanol and (c) ethanol are presented. There only very few nanobubbles are formed. Images (b) and (d) show the substrate after propanol and ethanol, respectively, have been flushed away by water. As a result, the density of nanobubbles is dramatically increased.

Clearly, in pure alcohol there are hardly any nanobubbles present on the substrate. However, flushing away the alcohols with water leads indeed to a dramatic increase of the nanobubble density. The density of nanobubbles after the alcohol-water-exchange is larger than for the standard procedure. With respect to a large nanobubble density ethanol gives better results than propanol. It has been suggested that the larger gas solubility in ethanol compared to water is responsible for the increased nanobubble density when ethanol is replaced by water [6]. However, we would like to point out that alternatively it has been suggested that the exothermic mixing of ethanol and water leads to the enhancement of nanobubble formation $[13,15]$. This is in accordance with the above presented observation that an increase of the substrate or liquid temperature increases the nanobubble density.

\subsubsection{Nanobubbles in $\mathrm{NaCl}$ solution}

Instead of pure water, sodium chloride $(\mathrm{NaCl})$ solution $(0.03 \mathrm{M})$ has been added on the surface. Figure 3.14 shows an AFM topography image of the surface under the $\mathrm{NaCl}$ solution. Nanobubbles are formed on the surface. The density and size of the nanobubbles formed in the $\mathrm{NaCl}$ solution is similar to those in the pure water.

\subsection{Conclusions}

We have presented AFM studies of surface nanobubbles on hydrophobilized silicon surfaces under varying conditions. With regard to detecting liquid-gas interface, a direct comparison between nanobubbles and microcavity that traps air inside under water is established: we imaged nanobubbles next to the microcavity (an actual manufactured microbubble in the vicinity of nanobubbles). The stability of nanobubbles has been examined: nanobubbles are very stable, at least on the timescale of hours. 


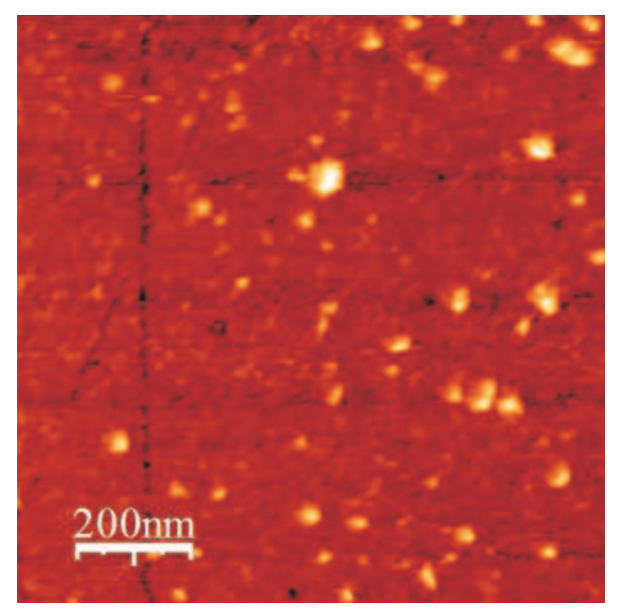

Figure 3.14: AFM tapping mode topography image (height range $8.1 \mathrm{~nm}$ ) of the surface immersed in $\mathrm{NaCl}(0.03 \mathrm{M})$ solution. Nanobubbles are formed with large density. Comparing with the water case, the density and size of nanobubbles formed in the $\mathrm{NaCl}$ solution are fairly the same.

The importance of the surface morphology was demonstrated by the movement of a nanobubble along a groove in the substrate and the tendency of nanobubbles to form (by means of heating the substrate) in the vicinity of grooves. In situ creation of nanobubbles allowed a direct comparison of nanobubble profiles and the underlying substrate topography. The growing and merging of nanobubbles have been imaged. We have provided evidence that the conditions during immersion are decisive, such that nanobubbles which are once formed due to favorable conditions during immersion, e.g. increased water temperature, stably remain on the surface even when the conditions become less favorable, e.g. water temperature decreases. In addition, pressurizing the water with $\mathrm{CO}_{2}$ gas has yielded a dependence of the nanobubbles on the gas concentration in the water, in terms of nanobubble size and density. Furthermore, we have shown that nanobubbles are sensitive to the cleaning procedure (alcohol prewashing process) applied to prepare the substrates. It has been found that addition of butanol to the water leads to a decrease of the nanobubbles in size. Finally, we reproduced the previously reported procedure to create surface nanobubbles through alcohol-water-exchange method, and instead of water $\mathrm{NaCl}$ solution has been added on the surface in which nanobubbles are formed as well with the same density and size. 


\section{References}

[1] Tyrrell, J. W. G.; Attard, P. Phys. Rev. Lett. 2001, 87, 176104.

[2] Ishida, N.; Inoue, T.; Miyahara, M.; Higoashitani, K. Langmuir 2000, $16,6377$.

[3] Holmberg, M.; Kühle, A.; Garnæs, J.; Mørch, K. A.; Boisen, A. Langmuir 2003, 19, 10510.

[4] Simonsen, A.C.; Hansen, P.L.; Klösgen, B. J. Colloid Interface Sci. 2004, 273, 291.

[5] Agrawal, A.; Park, J.; Ryu, D. Y.; Hammond, P. T.; Russel, T. P.; McKinley, G. H. Nano Lett. 2005, 5, 1751.

[6] Zhang, X. H.; Maeda, N.; Craig, V. S. J. Langmuir 2006, 22, 5025.

[7] Zhang, L.; Zhang, Y.; Zhang, X.; Li, Z.; Shen, G.; Ye, M.; Fan, C.; Fang, H.; Hu, J. Langmuir 2006, 22, 8109.

[8] Zhang, X. H.; Zhang, X. D.; Lou, S. T.; Zhang, Z. X.; Sun, J. L.; Hu, J. Langmuir 2004, 20, 3813.

[9] Agrawal, A.; McKinley, G. H. Mater. Res. Soc. Symp. Proc. 2006, 899E.

[10] Switkes, M.; Ruberti, J. W. Appl. Phys. Lett. 2004,84, 4759.

[11] Steitz, R.; Gutberlet, T.; Hauss, T.; Klösgen, B.; Krastev, R.; Schemmel, S.; Simonsen, A. C.; Findenegg, G. H. Langmuir 2003, 19, 2409.

[12] Bremond, N.; Arora, M.; Ohl, C. D.; Lohse, D. Phys. Rev. Lett. 2006, 96, 224501.

[13] Borkent, B. M.; Dammer, S. M.; Schönherr, H.; Vancso, G. J.; Lohse, D. Phys. Rev. Lett. 2007, 98, 204502.

[14] Bremond, N.; Arora, M.; Ohl, C. D.; Lohse, D. J. Phys.: Condens. Matter 2005, 17, S3603.

[15] Coffey, S. Rodd's chemistry of carbon compounds, 2nd edition, vol 1, part B; Elsevier, Amsterdam, 1965.

[16] Gaines, G. L. Insoluable monolayers at liquid/gas interfaces; Intersciene, John Wiley, New York, 1966. 


\section{4}

\section{Correlation between Geometry and Nanobubble Distribution on HOPG Surfaces}

We show in this Chapter that the distribution of nanobubbles is inhomogeneous on the (under-water) surface of highly orientated pyrolytic graphite (HOPG), reflecting the atomic steps: The formation of nanobubbles is strongly enhanced at the upper side of the atomic steps, i.e., the most hydrophobic area on the surface. In contrast, no nanobubbles are formed at the lower side of the steps, i.e., the most hydrophilic area. The width of this nanobubbble-free zone is approximately $20 \mathrm{~nm}$. We thus establish a correlation between surface topography and nanobubble formation. In addition, we show that the profile of nanobubbles is sensitive to the applied AFM tip-force, demonstrating the deformability of nanobubbles.

Published as: Shangjiong Yang, Stefan Kooij, Bene Poelsema, Detlef Lohse, and Harold Zandvliet, Europhysics Letters 2008, 81, 64006. 


\subsection{Introduction}

Experimental observations show that hydrophobic surfaces (contact angle $>90^{\circ}$ ) favor the formation of nanobubbles. Nevertheless, it has been demonstrated that even on hydrophilic surfaces (contact angle $<90^{\circ}$ ) nanobubbles can efficiently form if the surface is initially covered by ethanol which is subsequently replaced by water (the so-called ethanol-waterexchange process, for explanations see ref. $[1,2]$ and refs. therein). Also, it has been suggested that the majority of nanobubbles prefer to form in the vicinity of nanometer-deep grooves on the surface [2]. In addition, the nanoscopic contact angle of the nanobubbles (typically $\sim 170^{\circ}$ ) is much larger than the macroscopic contact angle on the surface (typically $\sim 100^{\circ}$ ) [1-3]. Following those observations, we conclude that nanobubbles are affected by surface properties, and their extraordinary shape, which is connected to their remarkably large nanoscopic contact angles, potentially is one of the keys to the understanding of their surprising stability. Therefore, more studies in order to correlate surface property and nanobubble formation are required.

The purpose of the work described in this Chapter is to elucidate this issue by performing atomic force microscopy (AFM) measurements of nanobubbles on highly orientated pyrolytic graphite (HOPG) surfaces, which consists of flat terraces separated by atomic steps, thus providing a well defined surface structure. The upper side of the steps comprises atoms with the lowest coordination, whereas the lower side of the steps consists of atoms that have the highest coordination, as compared to the terrace. Water molecules prefer to stick to sites where their local coordination is maximum, i.e., at the lower side of the steps. Therefore, the upper side of the steps is more hydrophobic and the lower side of the steps is more hydrophilic than an area on the middle of a terrace $[4,5]$. Hence, hydrophobicity varies in a well-defined way on a vicinal surface. Indeed, as shown in ref. [4], water droplets preferentially condensate on the lower side of the HOPG steps. We will show in this Chapter that also the formation of nanobubbles on HOPG is strongly correlated with the variation of hydrophobicity on the HOPG surface, namely, nanobubbles preferentially form on the upper side of the HOPG steps.

In addition, we studied how liquid-gas interface responds to the applied AFM tip force. AFM measurements at different tip forces are performed on a solid feature, a meniscus in a microcavity, and a surface nano- 
bubble. It is shown that the measurements on the meniscus and surface nanobubble are very sensitive to the tip force, demonstrating the deformability of nanobubbles.

\subsection{Experimental Section}

In the experiment, water is prepared by a Milli-Q Synthesis A10 system (Millipore SAS, France). Alcohols used, i.e., methanol, ethanol, 2-propanol, and butanol are of GR grade ( $>99.8 \%$, Merch KGaA, Germany). AFM measurements are done with a PicoSPM (Molecular Imaging, AZ, USA) operated in tapping mode. AFM operating parameters are as follows: scanning speed $4 \mu \mathrm{m} / \mathrm{s}$ both in air and in liquid; free amplitude $5 \mathrm{~V}$ in air, $400 \mathrm{mV}$ in liquid; set-point amplitude $3.5 \mathrm{~V}$ in air, $300 \mathrm{mV}$ in liquid. The resonance frequency is $75 \mathrm{kHz}$ in air, and $20 \mathrm{kHz}$ in liquid. AFM scanning is performed by a hydrophilic $\mathrm{Si}_{3} \mathrm{~N}_{4}$ ultra-sharp AFM tip (radius of curvature $<10 \mathrm{~nm}$, full tip cone angle $<30^{\circ}$, NSC18/AlBS, MikroMasch, France; rinsed with ethanol and pure water before use). The HOPG sample (HOPG ZYB/1.75, MikroMasch, France) with a freshly cleaved surface is placed in a metal fluid cell, and then the cell is inserted into the AFM. To efficiently yield nanobubbles on the surface, the ethanol-water-exchange process is applied, i.e., ethanol is first injected into the cell, which is subsequently replaced by the water being flushed in. Thereafter, AFM scanning starts. The experiment is carried out in a general lab environment with a temperature between 20 and $23^{\circ} \mathrm{C}$.

\subsection{Results and Discussions}

\subsubsection{Hydrophobicity varies at atomic steps of HOPG surface}

Figure 4.1(a) shows a 3-dimensional AFM topography image of a freshly cleaved HOPG surface in air (tapping mode). The scan size is $2 \mu \mathrm{m} \times 2 \mu \mathrm{m}$, the height range is $5.0 \mathrm{~nm}$, and the root mean square (RMS) roughness is $0.7 \mathrm{~nm}$. It confirms that HOPG consists of a train of flat terraces separated by steps of single, double, or triple atomic-step-height. These steps provide a variation in hydrophobicity on the surface, i.e., the upper side of the steps is the most hydrophobic while the lower side of the steps is the 

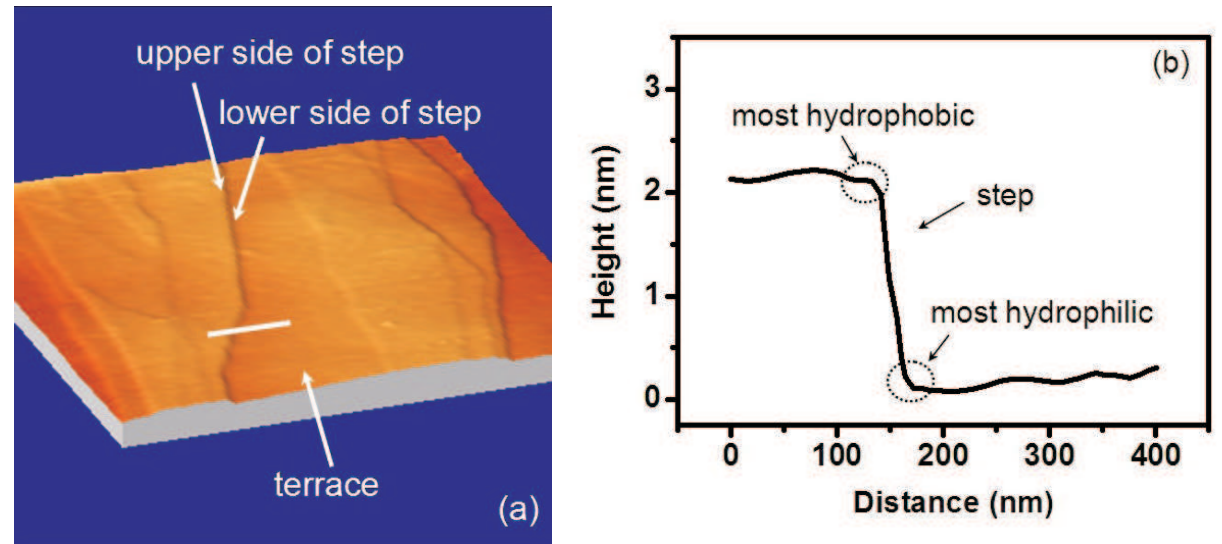

Figure 4.1: (a), 3-dimensional AFM topography image of freshly cleaved HOPG surface in air (tapping mode, scan size: $2 \mu \mathrm{m} \times 2 \mu \mathrm{m}$, height range: $5.0 \mathrm{~nm}$ ). The sample consists of a plane surface (RMS roughness: $0.7 \mathrm{~nm}$ ) which is traversed by steps of atomic layers $(0.3-2.0 \mathrm{~nm}$ in height). (b), cross-sectional profile corresponding to the line-cut crossing a step, as depicted in (a). These steps provide a variation in hydrophobicity on the surface: the upper side of the steps is the most hydrophobic area while the lower side of the steps is the most hydrophilic area, and the terrace is intermediate.

most hydrophilic, and the terrace is intermediate, as respectively pointed by the arrows in the image. Figure 4.1(b) shows the cross-sectional profile corresponding to the line-cut crossing a step, as depicted in Figure 4.1(a).

\subsubsection{Ethanol-water-exchange process sufficiently produces nano- bubbles on HOPG}

The HOPG surface has a relatively low hydrophobicity (advancing contact angle $=89.1^{\circ}$, as we measured), compared with the silane sample used in Chapter 3. Therefore the yield of nanobubbles on HOPG is low if the general experimental method is followed, namely, simply adding water drops on the surface, as shown in Figure 4.2(a): there are no nanobubbles visible in the image. Instead of water, pure alcohols have also been directly added onto the surface and then AFM measurements follow. Figure 4.2 shows the AFM images (height range $5.5 \mathrm{~nm}$ ) of HOPG surface under (b) methanol, (c)ethanol, (d)2-propanol, and (e)butanol. Clearly, no 

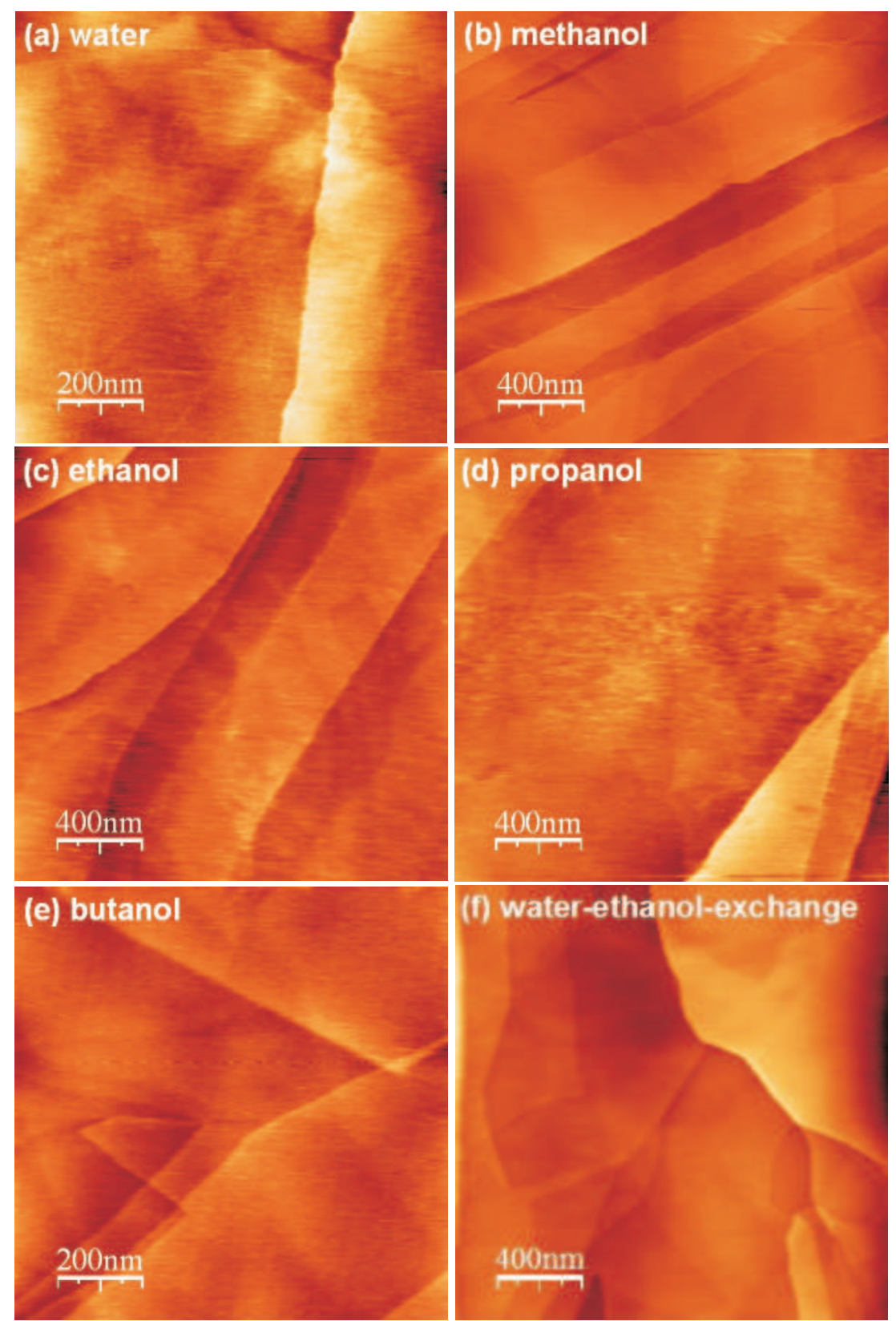

Figure 4.2: AFM topography image (tapping mode, height range $5.5 \mathrm{~nm}$ ) of HOPG surface under directly added (a) water, (b) methanol, (c) ethanol, (d) 2-propanol, (e) butanol, and (f) after the water-ethanol-exchange process. Clearly, no nanobubbles appear in the images. 
nanobubbles appear in the alcohols. As a contrast to the ethanol-waterexchange process, water-ethanol-exchange has also been performed, i.e., the surface is initially covered by water which is subsequently replaced by ethanol: no nanobubbles are formed either, as shown in Figure 4.2(f). Finally, after the ethanol-water-exchange process has taken place: nanobubbles form with a large density on the surface, as shown in Figure 4.3. It has been suggested that the larger gas solubility in ethanol compared to water is responsible for the increased nanobubble density when ethanol is replaced by water, which efficiently causes a local gas saturation at the liquid-solid interface [1]. Also, alternatively it has been suggested that the exothermic mixing of ethanol and water, which causes a dramatic temperature rising at the liquid-solid interface, leads to the enhancement of nanobubble formation $[2,6,7]$.

\subsubsection{Correlation between surface topography and nanobubble formation}

In Figure 4.3 the HOPG surface is shown under water, namely, after the ethanol-water-exchange process has taken place. Nanobubbles have been formed with a large density on the surface, (a) 2-dimensional; (b) 3-dimensional AFM topography image (tapping mode). The scan size is $2 \mu \mathrm{m} \times 2 \mu \mathrm{m}$ and the height range is $8.8 \mathrm{~nm}$. Atomic steps crossing the surface are visible. Note that the density of nanobubbles is significantly larger along the upper side (to the left) of the steps than on the remainder of the surface, whereas directly at the lower side (to the right) of the steps no nanobubbles are found. Figure 4.3(c) shows a zoom-in of the dashed square of Figure 4.3(a). A step crosses the surface from the upper-right to the lower-left: four nanobubbles are sitting on its upper side (see the white arrows). In contrast, no nanobubbles appear on its lower side (see the black arrows). In this particular region this bubble-free zone is approximately $70 \mathrm{~nm}$ wide.

To perspicuously highlight the inhomogeneous nanobubble distribution on the surface, the number density of nanobubbles is plotted as a function of the distance $d$ of the nanobubble to its nearest step. When the nanobubble is on the upper side of its nearest step, $d$ is positive, and when it is on the lower side of its nearest step, $d<0$. The statistics involves 350 nanobubbles. Figure 4.4 shows the graph for $d$ within the range $-250 \mathrm{~nm}$ to $250 \mathrm{~nm}$. Indeed, at the upper edge of the steps $(0<\mathrm{d}<20 \mathrm{~nm})$, where 


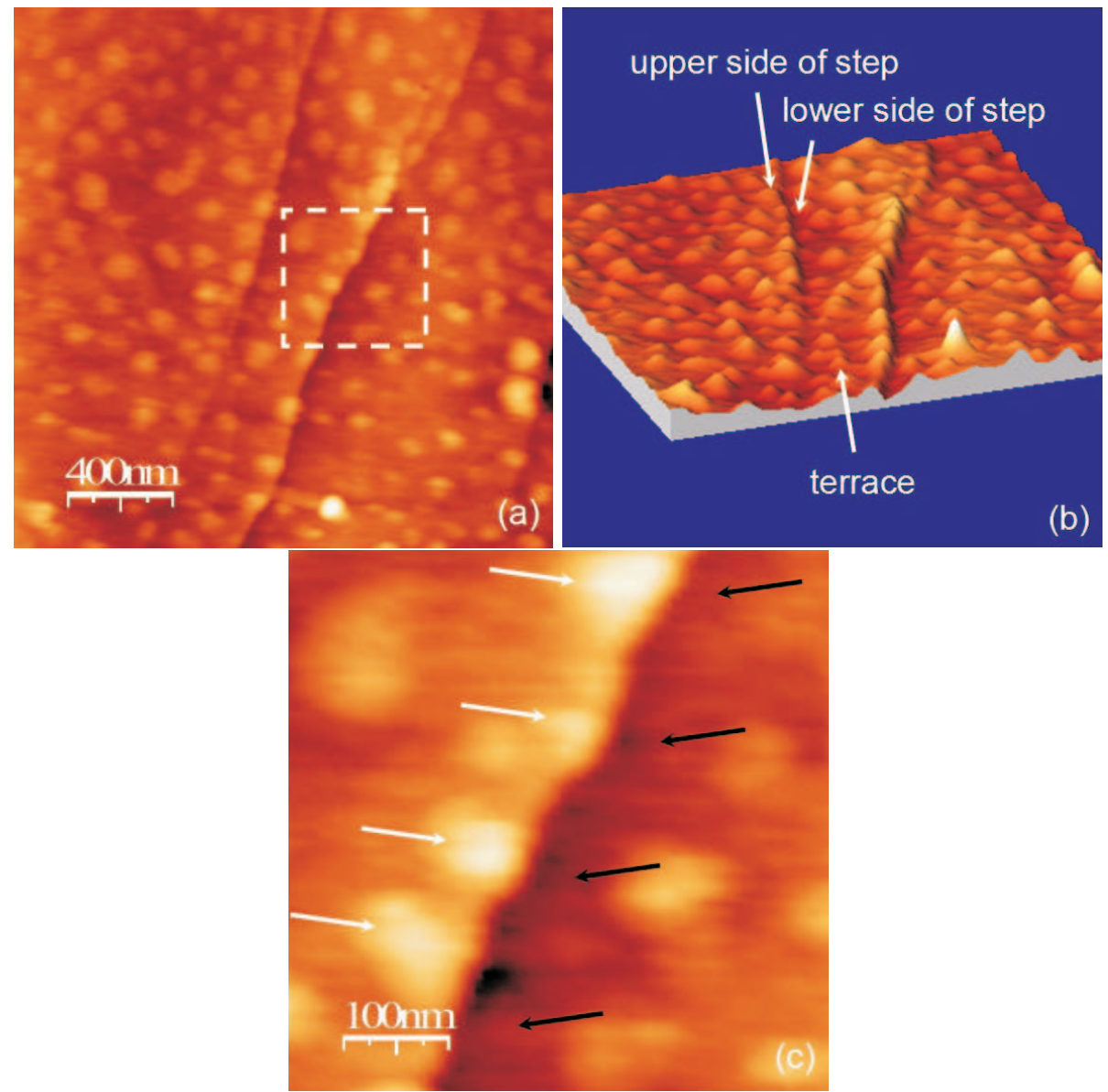

Figure 4.3: A typical AFM topography image of HOPG surface after the ethanol-water-exchange process has taken place (tapping mode, scan size: $2 \mu \mathrm{m} \times 2 \mu \mathrm{m}$, height range: $8.8 \mathrm{~nm}$ ). (a): 2-dimensional; (b): 3 dimensional; (c): zoom-in on the dashed square in (a). Nanobubbles form with a large density on the surface. Atomic steps are visible. We see that many nanobubbles are formed along the upper side (i.e., to the left) of the steps. In contrast, on the lower side (i.e., to the right) of the steps no nanobubbles are found. In (c) a step crosses the surface from upper-right to lower-left: four nanobubbles are sitting on its upper side (see white arrows), whereas no nanobubbles appear on its lower side (see black arrows). In this particular region this bubble-free zone is $\approx 70 \mathrm{~nm}$ wide. 


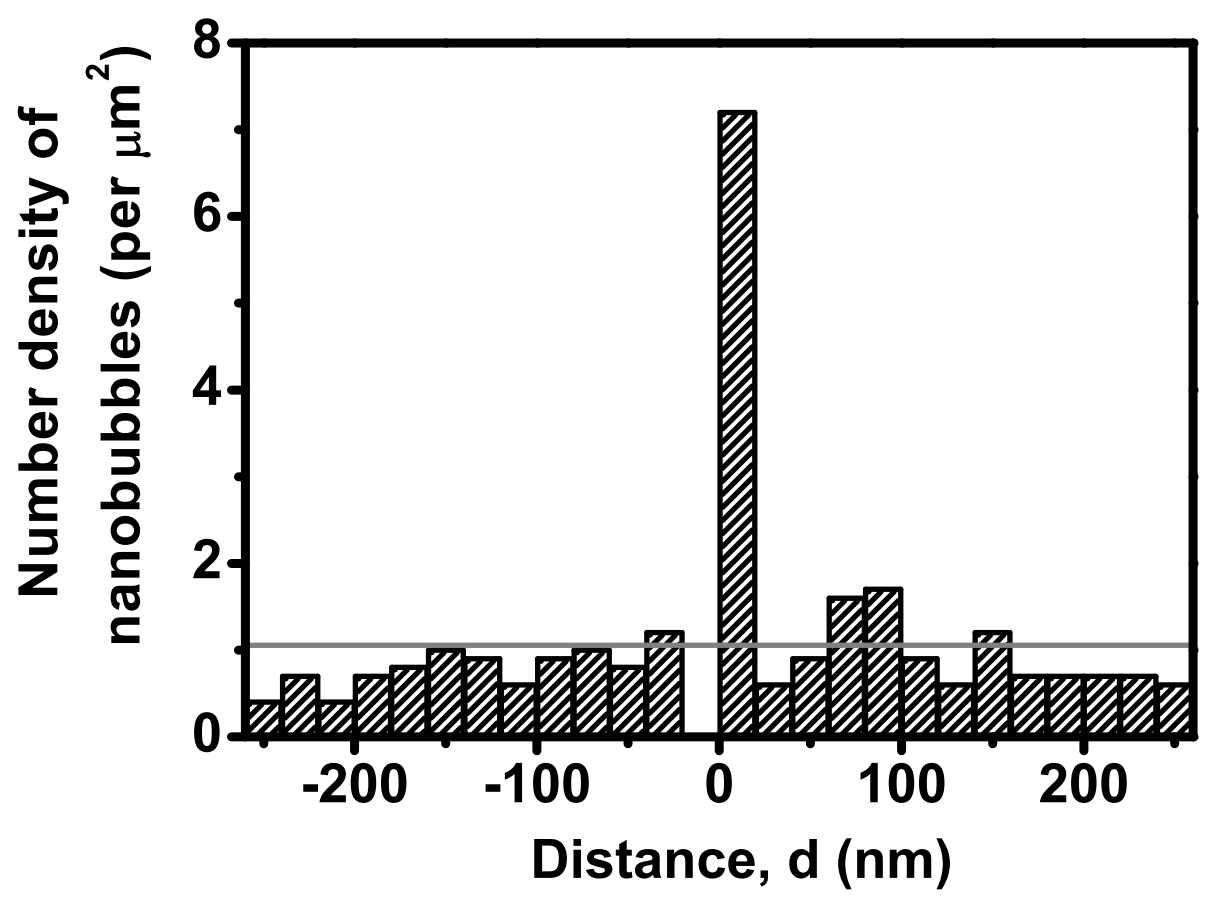

Figure 4.4: Number density of nanobubbles (per $\mu \mathrm{m}^{2}$ ) versus distance of nanobubble to its nearest step, $d$. When nanobubble is on the upper side of its nearest step, $d$ is positive, and when nanobubble is on the lower side of its nearest step, $d$ is negative. The graph clearly reveals the inhomogeneous bubble distribution around the steps. A nanobubblefree zone is shown on the lower side of the steps (the most hydrophilic area), which ranges from the step edge to approximately $20 \mathrm{~nm}$ away $(-20 \mathrm{~nm}<\mathrm{d}<0)$. In contrast, on the edge of the upper side of the steps (the most hydrophobic area, where $0<\mathrm{d}<20 \mathrm{~nm}$ ), nanobubbles are strongly favored. The grey horizontal line indicates the average number density value, which is $1.057 \mu \mathrm{m}^{-2}$. 
the surface is most hydrophobic, there is a considerable accumulation of nanobubbles: The density is strongly enhanced as compared to the values on the terrace far away from the steps, where the bubble density is more or less homogeneous. In contrast, at the lower side, for $-20 \mathrm{~nm}<\mathrm{d}<0 \mathrm{~nm}$, there is a bubble depletion zone. Indeed, as stated above, the lower sides of the steps are the most hydrophilic areas, thus disfavoring nanobubbles. Here we confirm that the length of this depletion zone is approximately $20 \mathrm{~nm}$. The grey horizontal line in the graph indicates the average number density value, $1.057 \mu \mathrm{m}^{-2}$.

\subsubsection{Deformability of nanobubbles}

In the final part of this Chapter we will address the relevance of the setpoint amplitude in AFM, completing earlier studies [1]. In the AFM tapping mode, the set-point amplitude $A_{s p}$ controls the effective interaction force $F$ between the tip and the surface, which is used as the feedback signal. The difference between the free amplitude $A_{0}$ and $A_{s p}$ is in first approximation proportional to F, i.e., $\left(A_{0}-A_{s p}\right) \propto F$. The plot in Figure 4.5(a) schematically shows the amplitude as a function of the distance between the cantilever and the surface. For lower set-point amplitude, the feedback and therewith the tip-surface interaction increases. Since nanobubbles are, in principle, a deformable entity, it is of great interest to study how nanobubbles depend on the applied force.

First of all, let's test how a solid feature responds to different interaction forces. The set-point amplitude ratio, i.e., $r_{s p}=A_{s p} / A_{0}$ is varied from $84 \%$ to $53 \%$, and the responding profile of the solid feature shows no dependence on the $r_{s p}$, as demonstrated by Figure 4.5(b). This is what can be expected for such a non-deformable solid feature. How about the liquid-gas interface? Discussed in Chapter 3.3.1, a microcavity traps air inside forming a meniscus. AFM cantilever is brought to scan above the meniscus at different $r_{s p}$. More interestingly, we allow the cantilever scanning to start from the solid edge of the cavity. Thus, it provides a direct comparison between the solid surface and liquid-gas interface, regarding to the response to the different $r_{s p}$. Figure 4.6(a) shows the AFM holdslow line-scan topography image of the meniscus and solid surface, at different $r_{s p}$. It is clearly shown that no considerable variations are on the solid surface, whereas the meniscus is very sensitive to the tip force change. The AFM tip pushes the liquid-gas interface downward with in- 

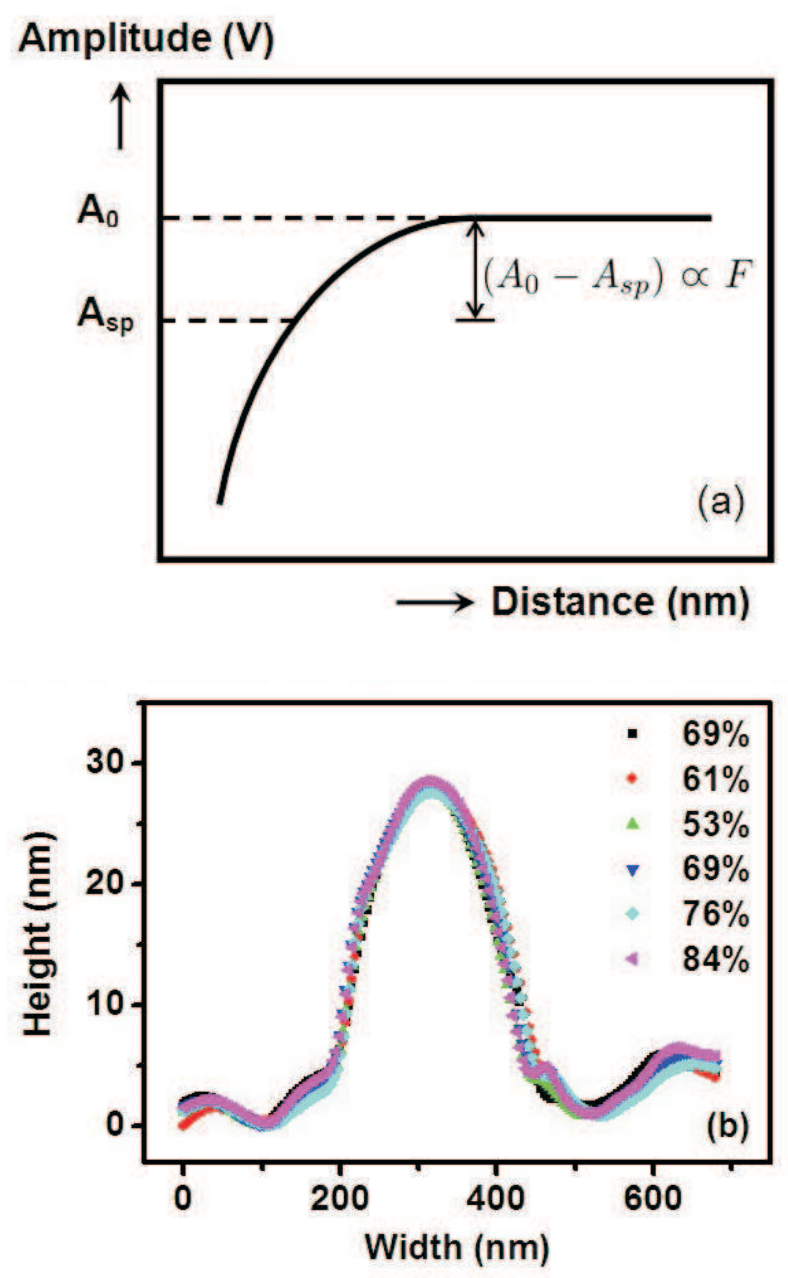

Figure 4.5: Plot (a) schematically shows the amplitude in AFM tapping mode as a function of the distance between cantilever and surface. Note that the difference between the free amplitude $A_{0}$ and the set-point amplitude $A_{s p}$ is proportional to the interaction force $F$ on surface. The setpoint amplitude ratio, i.e., $r_{s p}=A_{s p} / A_{0}$ is varied from $84 \%$ to $53 \%$ on a solid feature. Plot (b) reveals that the responding profile of a solid feature shows no dependence on the $r_{s p}$. 

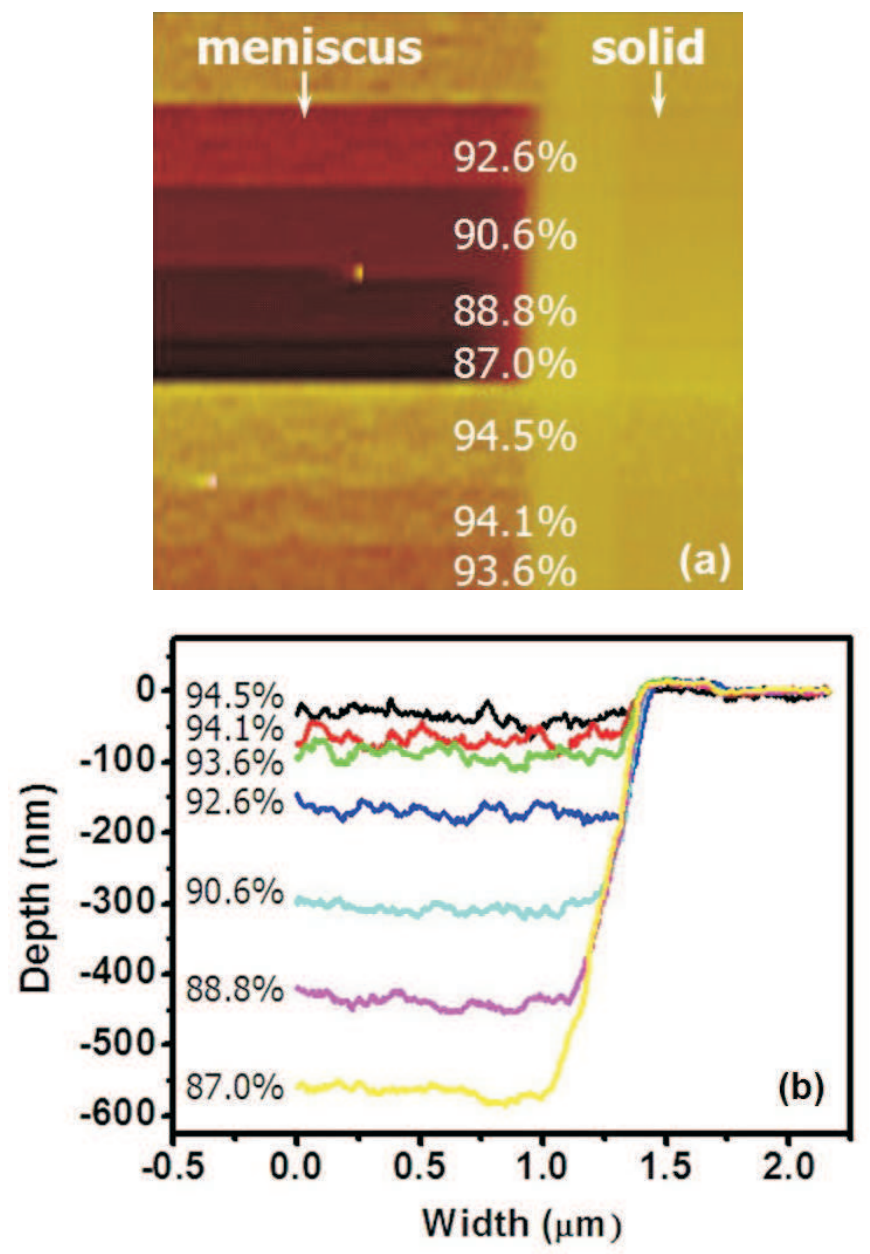

Figure 4.6: Panel (a) shows the AFM hold-slow line-scan topography image of on a microcavity where presents a meniscus and solid edge, at different $r_{s p}$. It is clearly shown that no considerable variations occur on the solid surface, whereas the meniscus is very sensitive to the tip force change. The AFM tip pushes the liquid-gas interface downward with increasing force, namely smaller $r_{s p}$. Plot (b) shows the relative crosssectional profile at each $r_{s p}$. (The AFM image is of the work in collaboration with Ms. Sissi de Beer, Physics of Complex Fluids, University of Twente.) 


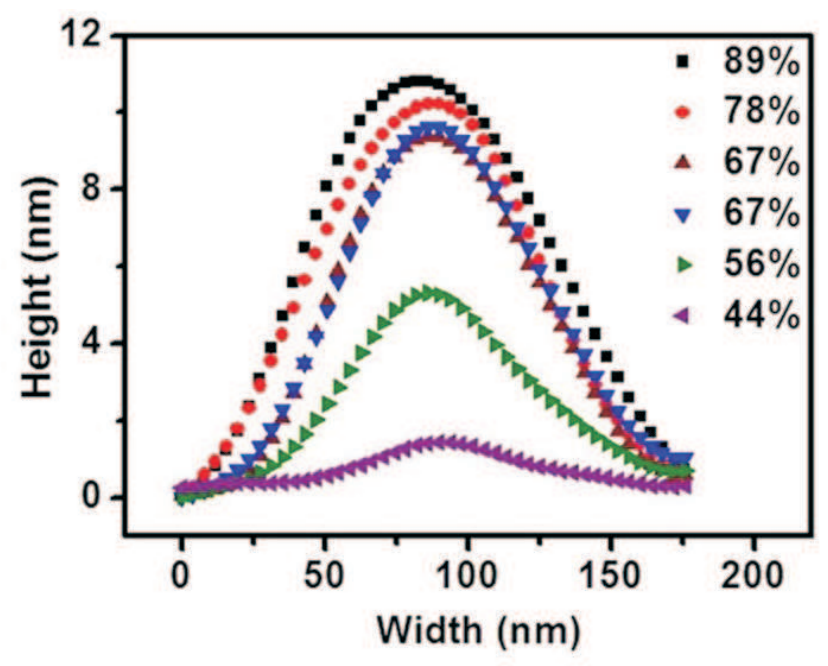

Figure 4.7: Plot shows the profiles of a nanobubble at different $r_{s p} \cdot r_{s p}$ is varied in the range of $89 \%-44 \%$, indicating an increase of $F$ on the nanobubble. The profile shrinks with increasing interaction strength, demonstrating the deformability of nanobubbles. Note that the shrinkage is relatively small from $89 \%$ to $67 \%$, while it becomes much larger from $67 \%$ to $44 \%$. To examine the repeatability, a second scan at $67 \%$ (down-triangle) is performed after the $44 \%$ scan and it is identical to the first $67 \%$ scan (up-triangle).

creasing force, namely smaller $r_{s p}$. Figure 4.6(b) shows the relative crosssectional profile of image (a) at each $r_{s p}$.

How about surface nanobubbles? Previously, in Zhang's work the setpoint amplitude ratio, $r_{s p}$ was varied from $92 \%$ to $74 \%$ and the responding profile of the nanobubble showed a very small variation [1]. In our study, $r_{s p}$ is varied over a considerably larger range, from $89 \%$ to $44 \%$, indicating a substantial increase of F. In Figure 4.7 we see that the profile of a nanobubble shrinks with increasing tip-surface interaction. Furthermore, the shrinkage is relatively small from $89 \%$ to $78 \%$, in agreement with Zhang's result; while the deformation becomes much larger below $67 \%$. At $44 \%$ the profile appears almost flat. To examine the repeatability, a second profile at $67 \%$ (down-triangle in the plot) is performed after the $44 \%$ scan and it is consistent with the first $67 \%$ (up-triangle). Note that the radius of curvature of the AFM tip $(<10 \mathrm{~nm})$ is much smaller than the width of the nanobubble $(\sim 175 \mathrm{~nm})$. We stress that the profiles revealed by the AFM 
scans, as shown in Figure 4.7, are not the actual shape of the nanobubble. The AFM tip only probes the local area where the tip interacts with the liquid-gas interface.

\subsection{Conclusions}

In conclusion, we have presented AFM studies of nanobubbles on HOPG surfaces where the hydrophobicity varies in a well-defined fashion due to the atomic steps. By means of the ethanol-water-exchange process, nanobubbles are formed with a large density. When simply adding pure water, methanol, ethanol, 2-propanol, butanol, or performing the waterethanol-exchange process, no nanobubbles are formed on HOPG surface. We have shown that the formation of nanobubbles is greatly enhanced at the upper side of the steps, which consists of the lowest coordinated atoms of the surface disfavoring water molecules (the most hydrophobic area). In contrast, no nanobubbles are formed on the lower side of the steps, which contains the highest coordinated atoms favoring water molecules (the most hydrophilic area). In addition, we have provided evidence that the mean length of this most hydrophilic area is approximately $20 \mathrm{~nm}$ from the steps. To our knowledge our study is the first correlation study between topography and surface nanobubble density and it only became possible by restricting us to the well-defined HOPG surfaces. Finally, we have shown that the profile of nanobubbles, as imaged by AFM, is sensitive to the applied interaction strength between the tip and the surface. The meniscus in a microcavity presents the same property. 


\section{References}

[1] Zhang, X. H.; Maeda, N.; Craig, V. S. J. Langmuir 2006, 22, 5025.

[2] Yang, S.; Dammer, S. M.; Bremond, N.; Zandvliet, H. J. W.; Kooij, E. S.; Lohse, D. Langmuir 2007, 23, 7072.

[3] Simonsen, A.C.; Hansen, P.L.; Klösgen, B. J.; Colloid Interface Sci. 2004, 273, 291.

[4] Zach, M.P.; Newberg, J.T.; Sierra, L.; Hemminger, J.C.; Penner, R.M. J. Phys. Chem. B 2003, 107, 5393.

[5] Zandvliet, H.J.W. Rev. Mod. Phys. 2000, 72, 593.

[6] Borkent, B. M.; Dammer, S. M.; Schönherr, H.; Vancso, G. J.; Lohse, D. Phys. Rev. Lett. 2007, 98, 204502.

[7] Coffey, S. Rodd's chemistry of carbon compounds, 2nd edition, vol 1, part B; Elsevier, Amsterdam, 1965. 


\section{5 Electrolytically Generated Nanobubbles on HOPG Surfaces}

Electrolysis of water is employed to produce surface nanobubbles on highly orientated pyrolytic graphite (HOPG) surfaces. Hydrogen (oxygen) nanobubbles are formed when the HOPG surface acts as negative (positive) electrode. Coverage and volume of the nanobubbles enhance with increasing voltage. The yield of hydrogen nanobubbles is much larger than the yield of oxygen nanobubbles. The growth of the individual nanobubbles during the electrolysis process is recorded in time with the help of AFM measurements and correlated with the total current. Both the size of the individual nanobubbles and the total current saturate after typical 1 minute; then the nanobubbles are in a dynamic equilibrium, meaning that they do not further grow, in spite of ongoing gas production and nonzero current. The surface area of nanobubbles shows a good correlation with the nanobubble volume growth rate, suggesting that either the electrolytic gas emerges directly at the nanobubbles' surface, or it emerges at the electrode's surface and then diffuses through the nano-

Langmuir, in press (2008) as: Shangjiong Yang, Peichun Tsai, Stefan Kooij, Andrea Prosperetti, Harold Zandvliet, and Detlef Lohse, Electrolytically Generated Nanobubbles on HOPG Surfaces. 
bubbles' surface. Moreover, the experiments reveal that the time constants of the current and the aspect ratio of nanobubbles are the same under all conditions. Replacement of pure water by water containing a small amount of sodium chloride $(0.01 \mathrm{M})$ allows for larger currents, but qualitatively gives the same results.

\subsection{Introduction}

As we have learned, studies on various physical aspects of nanobubbles have been increasingly undertaken in the last few years [1-17]. The solid surfaces employed include gold [7], polystyrene [6, 8], mica [10], silanehydrophobilized silicon wafer [11-13,16], and HOPG $[9,11,15]$. Most studies are done with highly purified water (Milli-Q), though some experiments have been done with alcohol [6] or dilute sulfuric acid solution [9]. Atomic force microcopy (AFM) in tapping mode is adopted in most experiments [4-15], while other techniques such as rapid cryofixation-freeze fracture [16], neutron reflectometry [17] and internal reflection infrared spectroscopy [18] have also been employed. Experimental observations show that nanobubbles are very stable, having an extraordinary shape with remarkably large aspect ratio $[11,13]$ which even further increases with decreasing nanobubble size [19]. The lifetime of nanobubbles shows a dependence on the gas type [18]. Besides the surface hydrophobicity, the spatial dimensions of the hydrophobic domains on the surface are crucial for the formation of nanobubbles [12]. It has also been reported that the formation of nanobubbles is related to surface nanostructures: the majority of nanobubbles prefer to form in the vicinity of nanometerdeep grooves [13] or on the upper side of atomic steps [15] on the surfaces. In addition, an increase of substrate temperature, water temperature, or gas concentration in water increases the density and size of nanobubbles $[10,13]$. These observations clearly reveal that the formation of nanobubbles is very sensitive to surface and liquid conditions. Yet, is there a simple method that leads to the controlled formation and growth of nanobubbles?

In electrochemical reactions, gas molecules are generated at electrode surfaces. Most studies have hitherto focused on mini- or micrometer sized bubbles, which are formed at and subsequently detach from the electrodes; see [20-25] and references therein. The formation of hydrogen microbubbles on a negative electrode has been witnessed by phase-contrast 


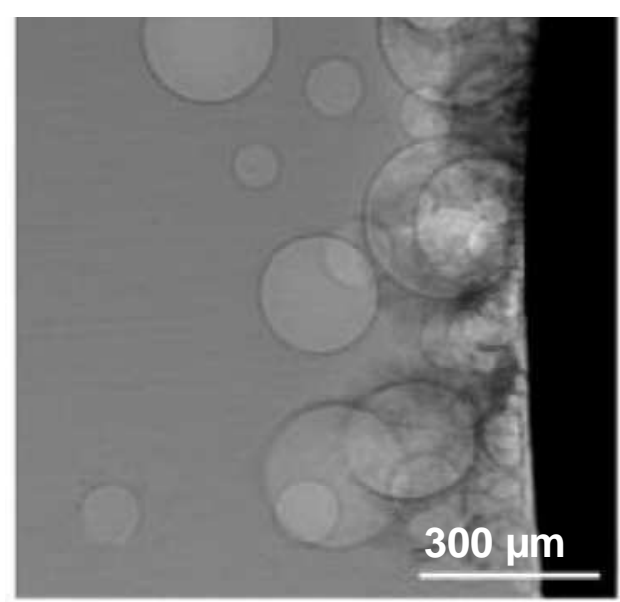

Figure 5.1: Phase-contrast microradiograph revealing the generation of micrometer sized hydrogen bubbles on an electrode that acts as cathode. Adopted from [20].

radiology with synchrotron radiation, as shown in Figure 5.1 [20]. The interest originates partly from the significant influence of the bubbles on reaction systems. E.g., convection caused by the evolution of electrogenerated microbubbles increases electrolyte flow and can enhance production processes [21]. The interest in electrochemically generated nanobubbles is more recent. It has been hypothesized that the existence of nanobubbles at electrode surfaces favors the formation of submicrometersized vaterite tubes in the electrolysis-induced mineralization [22]. Zhang et al. [9] confirmed that electrochemical generation of hydrogen induces the formation of nanobubbles on the electrode surface in sulfuric acid solution.

The work described in this Chapter is motivated by two issues: (i) Electrolysis of water is a reliable and controllable way to rapidly produce high local gas concentration at the electrode surfaces. Gas concentration significantly affects the formation of nanobubbles [13]. Electrolysis of water therefore is an easy method to control the appearance and growth of surface nanobubbles. This is demonstrated by performing AFM measurements of nanobubbles on an HOPG surface which acts as electrode. To reduce the effect of any possible impurities in the liquid, since nanobubbles are extremely sensitive to surfactants, ultraclean water (see below for qualification) is used as electrolyte. In addition, to test the repro- 
ducibility, an aqueous sodium chloride solution $(0.01 \mathrm{M})$ is also used. We study the bubble coverage, volume, size, and aspect ratio at different voltages. In addition, we show the real-time development of individual nanobubbles, before they finally achieve a dynamic equilibrium condition. Remarkably, the nanobubble's surface area and its volume growth are highly correlated, suggesting that either the electrolytic gas is produced at the whole surface of the nanobubbles, or it is generated at the electrode's surface and diffuses to the surface of nanobubbles. (ii) The second issue of this Chapter is to correlate geometric feature of the nanobubbles with the electric current that flows between the two electrodes. We find a good correlation between the aspect ratio of the nanobubbles and the current.

\subsection{Experimental Section}

The water is prepared by a Milli-Q Synthesis A10 system (Millipore SAS, France) and then degassed at 1 mbar for 4 hours. AFM measurements are done with a PicoSPM (Molecular Imaging, AZ USA) operated in tapping mode. Excitation of the tip vibration is done acoustically, using a small piezo-element in the tip holder. The AFM operating parameters in water are as follows: scanning speed $6 \mu \mathrm{m} / \mathrm{s}$; free amplitude $400 \mathrm{mV}$; set-point amplitude $300 \mathrm{mV}$; resonance frequency $20 \mathrm{kHz}$. AFM scanning is performed by a hydrophilic $\mathrm{Si}_{3} \mathrm{~N}_{4}$ ultra-sharp AFM tip (radius of curvature $<10 \mathrm{~nm}$, full tip cone angle $<30^{\circ}$, NSC18/AlBS, MikroMasch, France; rinsed with ethanol and pure water before use). An HOPG sample (HOPG ZYB/1.75, size $10 \mathrm{~mm} \times 10 \mathrm{~mm}$, MikroMasch, France) with a freshly cleaved surface placed on a copper plate is used as nanobubble forming surface and at the same time as one of the electrodes. A platinum wire (diameter $0.25 \mathrm{~mm}$ ) placed next to the AFM cantilever is used as the other electrode. The copper plate and the platinum wire are connected to an electrometer (Picoammeter/Voltage Source 6478, Keithley Instruments Inc., OH USA). After a water drop (volume $0.33 \mathrm{ml}-0.40 \mathrm{ml}$ ) is placed on the HOPG surface and the desired voltage is imposed, AFM scanning process is started immediately. Figure 5.2(a) shows a sketch of the setup.

When the HOPG sample acts as the negative electrode (cathode), the reduction process of water leads to the formation of hydrogen molecules on the HOPG surface, $2 \mathrm{H}_{2} \mathrm{O}(\mathrm{l})+2 e^{-} \rightarrow \mathrm{H}_{2}(\mathrm{~g})+2 \mathrm{OH}^{-}(\mathrm{aq})$. Oxygen molecules are produced on the HOPG surface when the HOPG sample is 

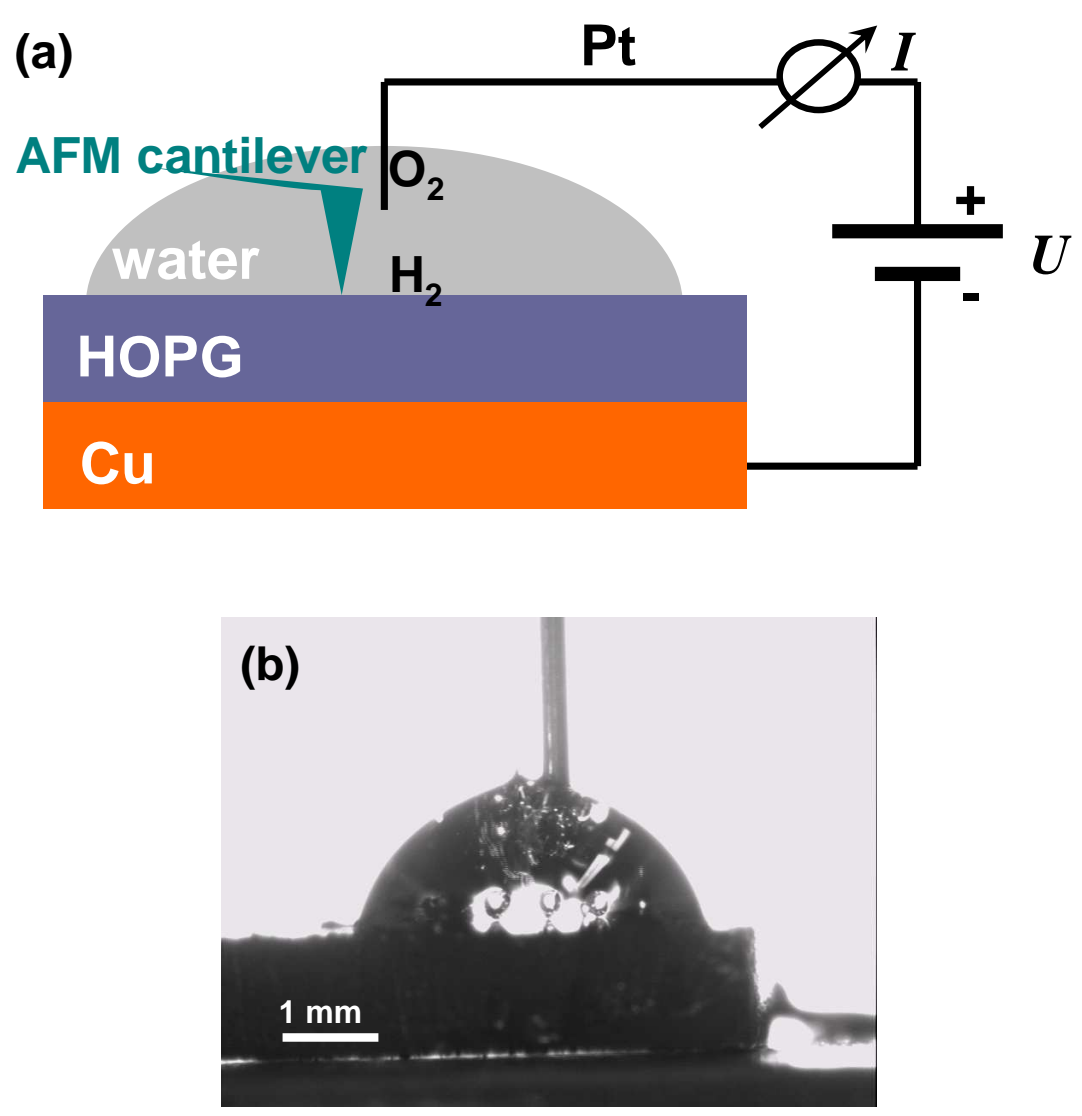

Figure 5.2: (a) Sketch describing our experimental setup. The HOPG sample is placed on a copper plate. A platinum wire of diameter $0.25 \mathrm{~mm}$ is set ( $\sim 2 \mathrm{~mm}$ away) next to the AFM cantilever. The copper plate and the platinum wire are connected to a power source supplying the voltage $U$ (the electrometer). The platinum wire and the HOPG surface act as the electrodes. The current $I$ is measured with a high precision amperemeter. When the HOPG surface is used as the negative (positive) electrode, water reduction (oxidation) process takes place producing hydrogen (oxygen) molecules on the HOPG surface. (b) a CCD camera image showing the generation of micrometer sized bubbles on both the HOPG surface (cathode) and the platinum wire (anode) of our setup under an electric potential of $10 \mathrm{~V}$. 
switched to be the positive electrode (anode) and therefore the oxidation process of water on the surface leads to oxygen molecules, $2 \mathrm{H}_{2} \mathrm{O}(\mathrm{l}) \rightarrow$ $\mathrm{O}_{2}(\mathrm{~g})+4 \mathrm{e}^{-}+4 \mathrm{H}^{+}(\mathrm{aq})$. Figure 5.2(b) shows a CCD camera picture of our setup under electrolysis process at $10 \mathrm{~V}$; numbers of microbubbles are continuously generated at both electrodes. The experiments are carried out in a standard lab environment with a temperature between 20 and $23^{\circ} \mathrm{C}$. The temperature change of the HOPG sample during the measurements is less than $0.1 \mathrm{~K}$.

\subsection{Results and Discussions}

\subsubsection{Nanobubbles by electrolysis of water: dependence on ap- plied voltage and gas type}

Previous experimental results show that no nanobubbles are formed on HOPG surfaces unless the so-called ethanol-water-exchange step is carried out $[11,15]$. This is due to the hydrophilic nature of the surface (macroscopic contact angle $<90^{\circ}$ ) that disfavors the attachment of surface bubbles. Electrolysis of water can be a robust method for a sufficient yield of nanobubbles on HOPG [9]. AFM measurements by tapping mode are performed on the HOPG surface.

Figure 5.3 shows the topography images of the HOPG surface used as cathode with different applied voltages: (a) $1 \mathrm{~V}$, (b) $1.5 \mathrm{~V}$, (c) $2 \mathrm{~V}$, (d) $2.5 \mathrm{~V}$, (e) $0 \mathrm{~V}$, (f) $3 \mathrm{~V}$, (g) $3.5 \mathrm{~V}$, (h) $4 \mathrm{~V}$; the height range for each image is (a-b) $42 \mathrm{~nm}$, (c-e) $50.6 \mathrm{~nm}$, (f) $61.2 \mathrm{~nm}$, and (g-h) $115.5 \mathrm{~nm}$. The images are recorded continuously from (a) to (h), with a scanning time of $8.5 \mathrm{~min}$ per image. Nanobubbles (hydrogen) form with varying density at different voltages. The atomic steps of HOPG are visible when the nanobubble coverage is low and thus act as a good reference position at the nanoscale when conducting AFM measurements. The formation of nanobubbles increases tremendously when increasing the voltage from 1.5 to $2 \mathrm{~V}$. Figure 5.3(e) reveals that the nanobubbles remain stable even when the voltage has been switched off from $2.5 \mathrm{~V}$ (d). This demonstrates the robust stability of nanobubbles, which is similar to the previous finding that the heating-water-generated nanobubbles do not disappear when the water is cooled down [13]. At the higher voltages, nanobubbles cover the entire surface with much larger individual sizes, see Figure 5.3(f-h). Growth 

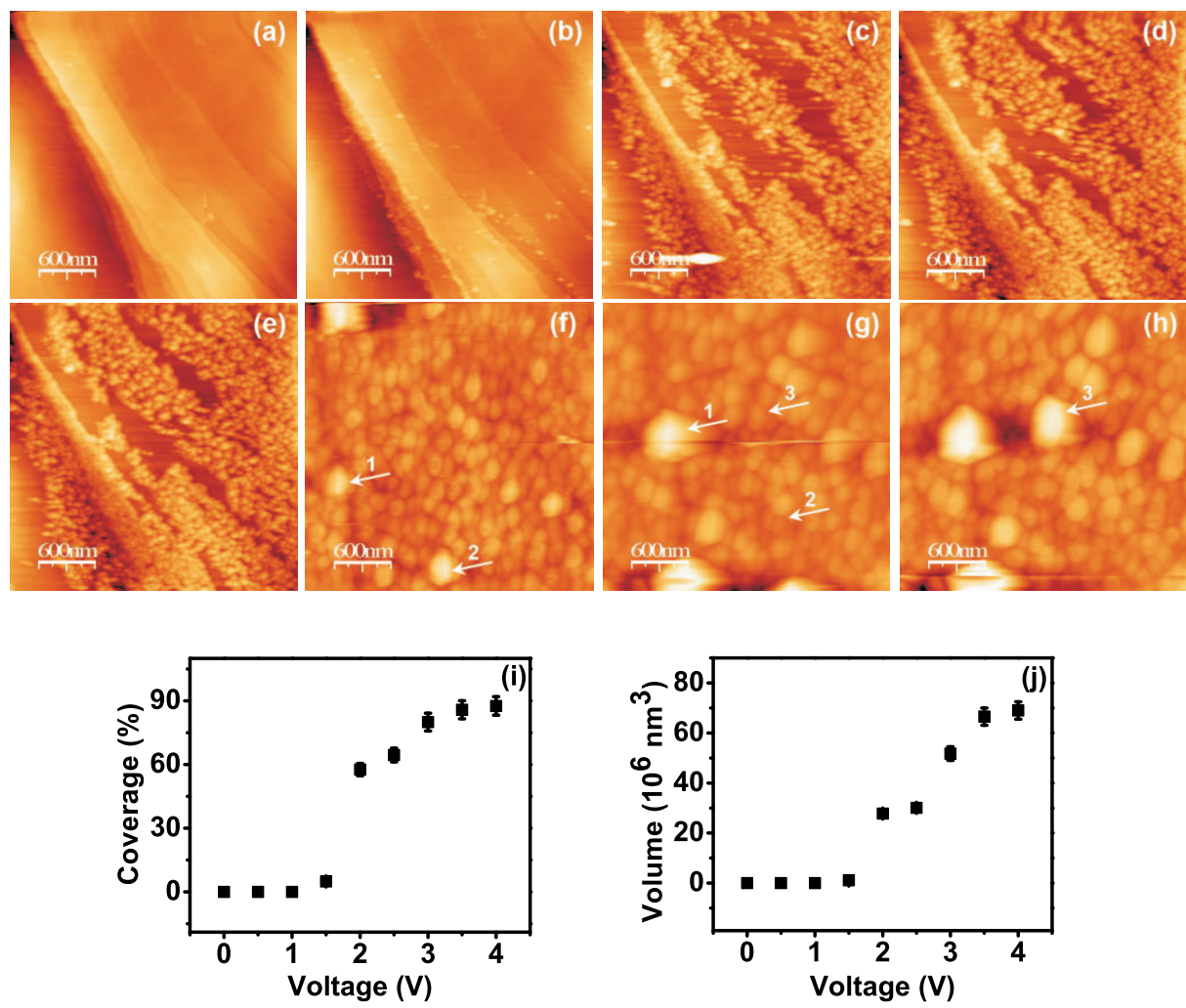

Figure 5.3: AFM (tapping mode) topography images of HOPG surface (under water) as cathode at different voltages: (a) $1 \mathrm{~V}$, (b) $1.5 \mathrm{~V}$, (c) $2 \mathrm{~V}$, (d) $2.5 \mathrm{~V}$, (e) $0 \mathrm{~V}$, (f) $3 \mathrm{~V}$, (g) $3.5 \mathrm{~V}$, and (h) $4 \mathrm{~V}$ (height range: (a-b) $42 \mathrm{~nm}$, (ce) $50.6 \mathrm{~nm}$, (f) $61.2 \mathrm{~nm}$, (g-h) $115.5 \mathrm{~nm}$ ). The scanning time per image is $8.5 \mathrm{~min}$ and the images are taken in a sequence from (a) to (h). Hydrogen nanobubbles are produced on the surface. When the nanobubble coverage is low, the atomic steps traversing the HOPG surface are visible. The formation of nanobubbles increases tremendously when increasing the voltage from 1.5 to $2 \mathrm{~V}$. In (e) the voltage has been switched off, while the nanobubbles remain stable. In (f-h), at higher voltages nanobubbles cover the entire surface with much larger individual sizes. Nanobubbles growing (marked by arrow 1 and 3) or detaching (marked by arrow 2) are observed. The dependence of nanobubble coverage and volume upon the applied voltage is shown as plot (i) and (j) (error bar $\pm 5 \%$ ), respectively. With increasing voltage, more hydrogen molecules are produced at the cathode (HOPG surface), enhancing the local gas concentration. This results in more and larger nanobubbles, as revealed by (i) and (j). 
and detachment of nanobubbles are observed under the higher electric potentials; the examples are marked by arrows in the images. The dependence of nanobubble coverage and volume on the applied voltage is respectively depicted in Figure 5.3(i) and (j) (error bar $\pm 5 \%$ ). With increasing voltage, more hydrogen molecules are produced at the cathode (HOPG surface), enhancing the local gas concentration. This leads to an increase in the coverage and volume of the nanobubbles, as revealed by plot (i) and (j) in Figure 5.3. At high voltages, i.e., 4.5 or $5 \mathrm{~V}$, microbubbles developing at the HOPG surface can already be monitored by an optical camera. The evolution of these microbubbles ruins the AFM scanning process by interfering with the vibrating AFM cantilever.

AFM topography images of HOPG surface used as anode are shown in Figure 5.4. Different voltages are applied: (a) $1 \mathrm{~V}$, (b) $1.5 \mathrm{~V}$, (c) $2 \mathrm{~V}$, (d) $2.5 \mathrm{~V}$, (e) $3 \mathrm{~V}$, (f) $3.5 \mathrm{~V}$, (g) $4 \mathrm{~V}$ (height range: (a) $12 \mathrm{~nm}$, (b-g) $35 \mathrm{~nm}$ ). The scanning time of each image is $8.5 \mathrm{~min}$. The images are taken in succession from (a) to (g) without any delay. Oxygen nanobubbles form on the surface. Comparing to the hydrogen case in Figure 5.3, the production of nanobubbles in Figure 5.4 is much smaller. We suggest that this is due to the considerable difference in the solubility of oxygen and hydrogen in water (at $20{ }^{\circ} \mathrm{C}$ the solubility of oxygen is $\sim 2$ times higher than that of hydrogen), as well as to the different production rate during the electrolysis, $\mathrm{H}_{2}: \mathrm{O}_{2}=2: 1$. The nanobubble coverage and volume are plotted as functions of the applied voltage, respectively, in Figure 5.4(h) and (i) (error bar $\pm 5 \%)$. For both hydrogen and oxygen, the plots in Figure 5.3(i-j) and 5.4(h-i) reveal a threshold and saturation of the nanobubble formation in dependence of the applied voltage.

The coverage and volume values presented in Figure 5.3(i-j) and 5.4(hi) are calculated by setting an appropriate hight threshold $z$ to extract nanobubbles. This is illustrated by the example in Figure 5.5. AFM (tapping mode) topography images (height range $27.2 \mathrm{~nm}$ ) of hydrogen surface nanobubbles are shown with different thresholds $z$ applied for the identification of surface nanobubbles: (a) $z=0 \mathrm{~nm}$, (b) $z=6 \mathrm{~nm}$, (c) $z=7$ $\mathrm{nm}$, (d) $z=8 \mathrm{~nm}$, (e) $z=9 \mathrm{~nm}$, (f) $z=10 \mathrm{~nm}$, and (g) $z=14 \mathrm{~nm}$. The principle is sketched in Figure 5.5(h). Areas below the threshold are represented as blue, whereas areas above are shown as yellowish depending on the height. The fraction of the latter area is shown in Figure 5.5(i) as function of the threshold $z$. The curve shows a pronounced shape. We take the value at the end of the straight shape region (marked by an arrow), where $z=9 \mathrm{~nm}$ presenting a nanobubble identification as shown in image (e), as 

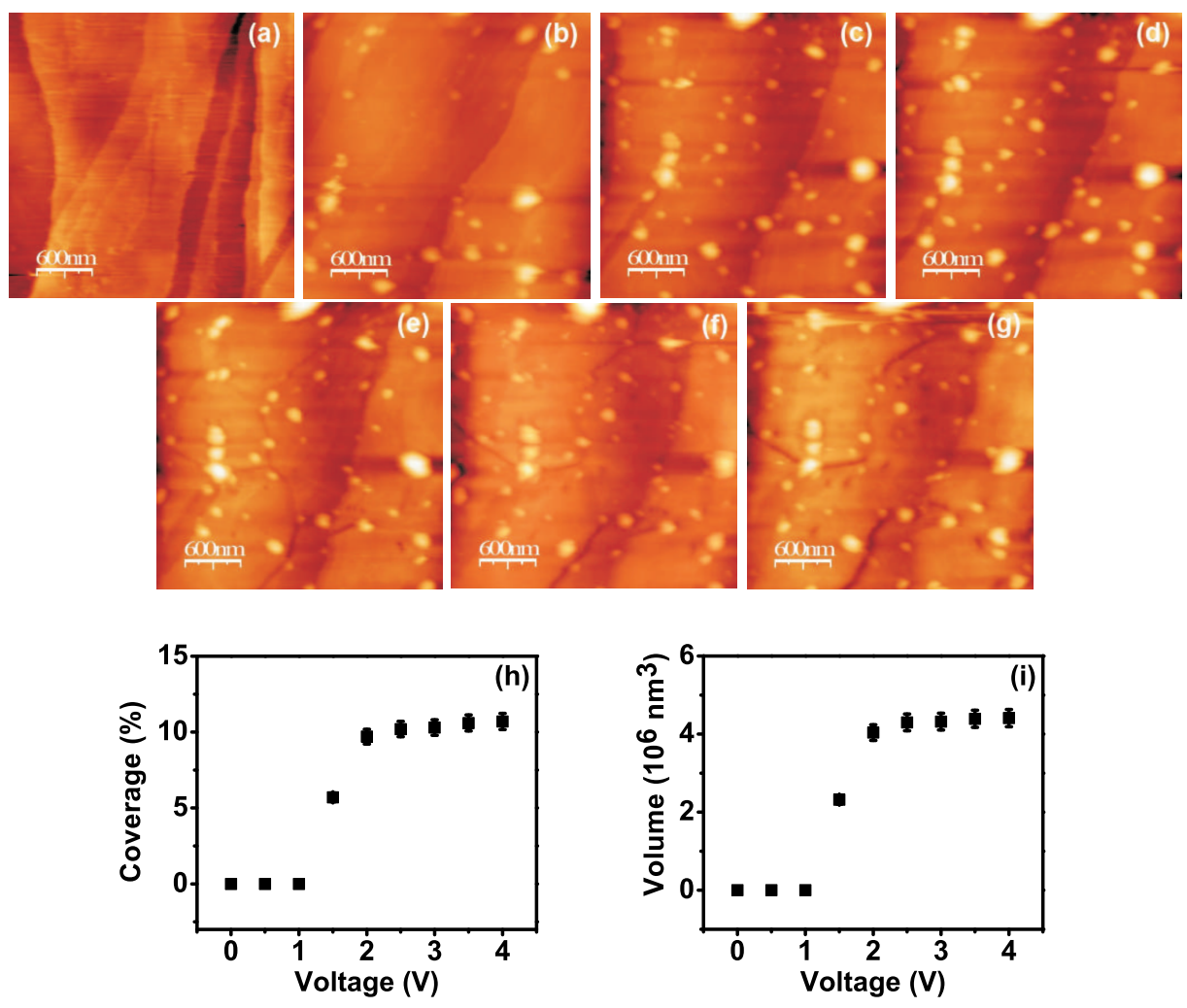

Figure 5.4: AFM (tapping mode) topography images of HOPG surface (under water) as anode at different voltages: (a) $1 \mathrm{~V}$, (b) $1.5 \mathrm{~V}$, (c) $2 \mathrm{~V}$, (d) $2.5 \mathrm{~V}$, (e) $3 \mathrm{~V}$, (f) $3.5 \mathrm{~V}$, and (g) $4 \mathrm{~V}$ (height range: (a) $12 \mathrm{~nm}$, (b-g) $35 \mathrm{~nm}$ ). Again, the atomic steps of HOPG surface are visible. Images are recorded continuously from (a) to (g), with a scanning time of $8.5 \mathrm{~min}$ per image. Nanobubbles (oxygen) are formed on the surface. Comparing to the hydrogen case in Figure 5.3, the number and volume of the produced oxygen nanobubbles is much smaller. This is presumably due to: i) the considerable difference of solubility in water between oxygen and hydrogen (oxygen's solubility is $\sim 2$ times higher than hydrogen's at $20^{\circ} \mathrm{C}$ ); ii) the difference in the production rate during the electrolysis, $\mathrm{H}_{2}: \mathrm{O}_{2}=2: 1$. Plot (h) and (i) show coverage and volume of the nanobubbles as a function of the imposed voltage, respectively (error bar $\pm 5 \%$ ). 


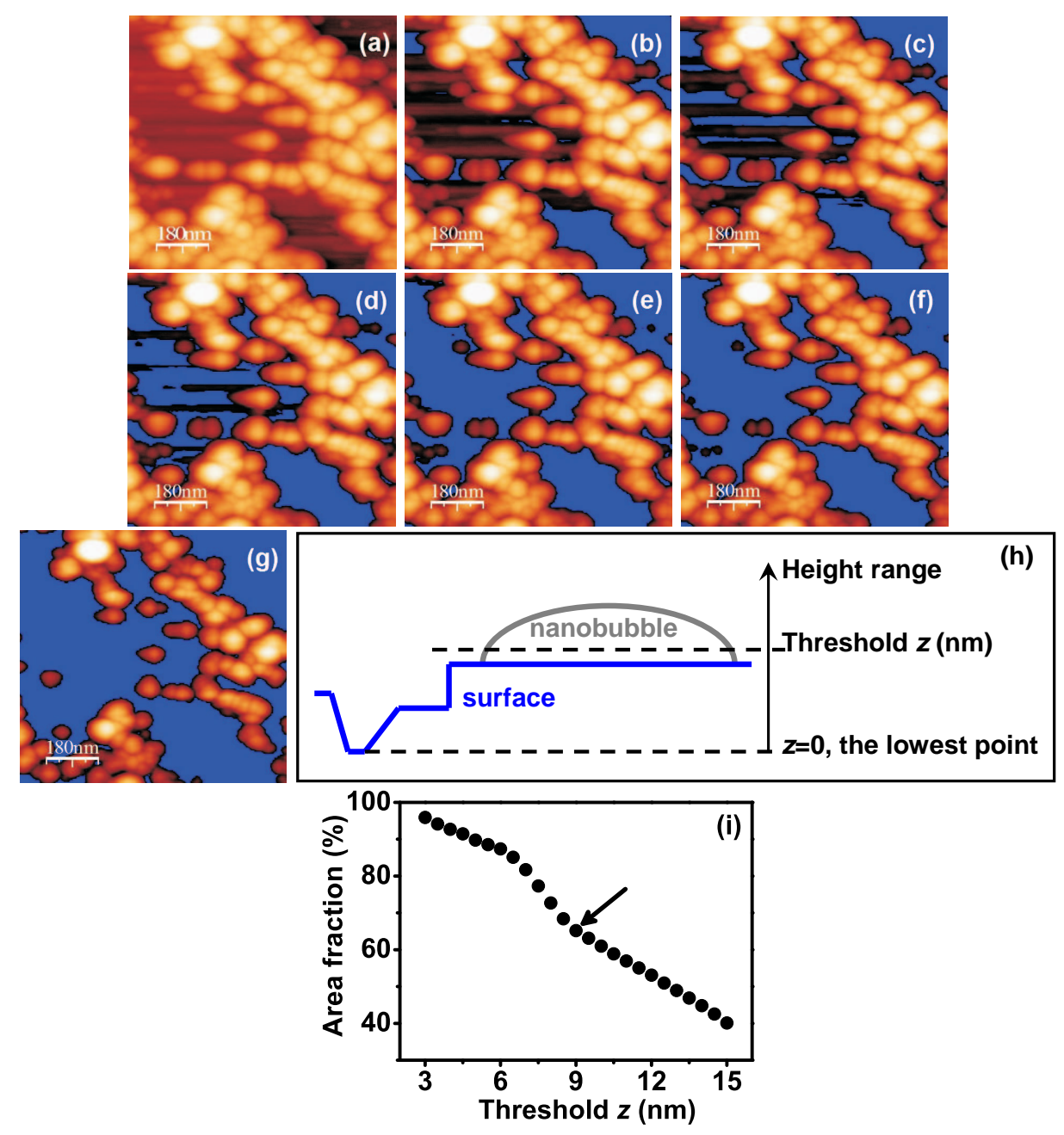

Figure 5.5: Tapping mode AFM topography images (height range $27.2 \mathrm{~nm}$ ) of hydrogen surface nanobubbles, when different thresholds $z$ are applied for the identification of surface nanobubbles: (a) $z=0 \mathrm{~nm}$, (b) $z=6 \mathrm{~nm}$, (c) $z=7 \mathrm{~nm}$, (d) $z=8 \mathrm{~nm}$, (e) $z=9 \mathrm{~nm}$, (f) $z=10 \mathrm{~nm}$, and (g) $z=14 \mathrm{~nm}$. Sketch (h) describes the principle. Areas below this threshold are mashed as blue, while areas above, depending on the height, as yellowish. The fraction of the latter area is shown in (i) as function of the threshold $z$. That curve shows a pronounced shape. We take the end of the straight shape region (see arrow and $z=9 \mathrm{~nm}$ ) towards smaller $z$ as estimate for the nanobubble coverage. 
estimate for the nanobubble coverage and volume statistics.

\subsubsection{Nanobubbles in dynamic equilibrium}

During the experiments, each chosen voltage is continuously applied while performing the AFM measurements shown in Figure 5.3 and 5.4. The constant voltage results in continuous charge flux through the system. Under such a condition, one may expect that surface nanobubbles would constantly accumulate on the electrode surfaces. However, our AFM images (Figure 5.3 and 5.4), taken after a certain transient time, show stationary nanobubbles of certain sizes. In other words, electrolytically generated nanobubbles experience a saturation in their development.

This suggests that the nanobubbles are in a dynamic equilibrium state. There are gas-flows into and out of the nanobubbles simultaneously, which balance each other allowing for a constant volume. When the inflow overwhelms the outflow, nanobubbles start to grow. This happens when the voltage is increased, producing more charges and leading to a larger gas flow into the nanobubbles, thus breaking the previous balance between the inflow and the outflow, and consequently causing the nanobubbles to grow. As the nanobubbles grow, the outflow starts to increase till it reaches a new equilibrium state with the inflow. The nanobubbles then again remain in a stable condition.

In order to quantify the growing process of the nanobubbles, we focus on a number of individual nanobubbles and measure the evolution of various geometric properties such as width, height, aspect ratio, etc. In addition and in parallel, we measure the global current as function of time (shown in the following sections). The electric current decays as the nanobubbles grow. This decrease in current, which reduces the amount of gas produced on the surface, effectively decreases the inflow to the nanobubbles. This of course helps to reach a new dynamic equilibrium state, but we stress again that the current is nonzero in the saturated state. The data of the current as function of time and the nanobubble development show saturation on the same time scale. At the saturated state, the nanobubble growth terminates, whereas the saturated current is nonzero. This observation clearly suggests the existence of a dynamic equilibrium of the nanobubbles. 


\subsubsection{Time evolution of nanobubbles}

The appearance of nanobubbles can easily be controlled by an increase of the voltage, as revealed in Figure 5.3 and 5.4. Thus, we can capture the dynamics of the nanobubble growth by operating the AFM tip to repeatedly scan along a fixed straight line on the surface over the time of the electrolysis. With this method we perspicuously quantify the evolution of the nanobubbles at the moment of increasing voltage. The measurements are shown in Figure 5.6.

During the experiment, we first start the AFM scan over one line on the HOPG surface, and then we apply the desired voltage to generate surface nanobubbles - meanwhile the AFM scan is continuously running. The time when we apply the voltage is taken as 0. Each AFM line-scan takes 1 sec; the profile of the developing nanobubble is continuously recorded. Figure 5.6(a) presents the profiles of a nanobubble generated with $1 \mathrm{~V}$ and the adjacent substrate surface (HOPG, as cathode) at different time with interval of $10 \mathrm{sec}$. Plot (b) exhibits the dynamics of another nanobubble generated at $2 \mathrm{~V}$. It is clearly shown that the nanobubbles start to grow continuously immediately after their appearance on the surface; this is also demonstrated by the nanobubble area $v$ s. time plots in Figure 5.7 and 5.8. Note that the growth terminates after $70 \mathrm{sec}$ for plot (a) and after $40 \mathrm{sec}$ for plot (b) in Figure 5.6. The nanobubbles then remain stable, although the voltage is still applied and the current is nonzero. The stabilized nanobubble in Figure 5.6(a) is approximately $200 \mathrm{~nm}$ in width and $5 \mathrm{~nm}$ in height. Interestingly, the measurements show that the nanobubbles grow with a faster rate in height than in width. The good agreement in the topography among the profiles of the adjacent HOPG surface at different times reveals that the AFM measuring is not considerably perturbed by the electrolysis process or the emergence of the nanobubbles, the profiles of the nanobubble therefore can be compared.

From the nanobubble profiles recorded by the AFM scan, we extract the width and height values of the nanobubbles at different times. Note that the AFM scan does not necessarily cross the center (the maximum width and height) of each nanobubble. Therefore the extracted width and height values may be lower than the maximum values. By assuming the shape of nanobubbles as a spherical cap, we estimate the surface area of a nanobubble as $\pi w^{2} / 4$ using the extracted width $w$. In a corresponding way we estimate the volume growth rate of a nanobubble, which as well as the surface area is then plotted as a function of time, shown in Fig- 

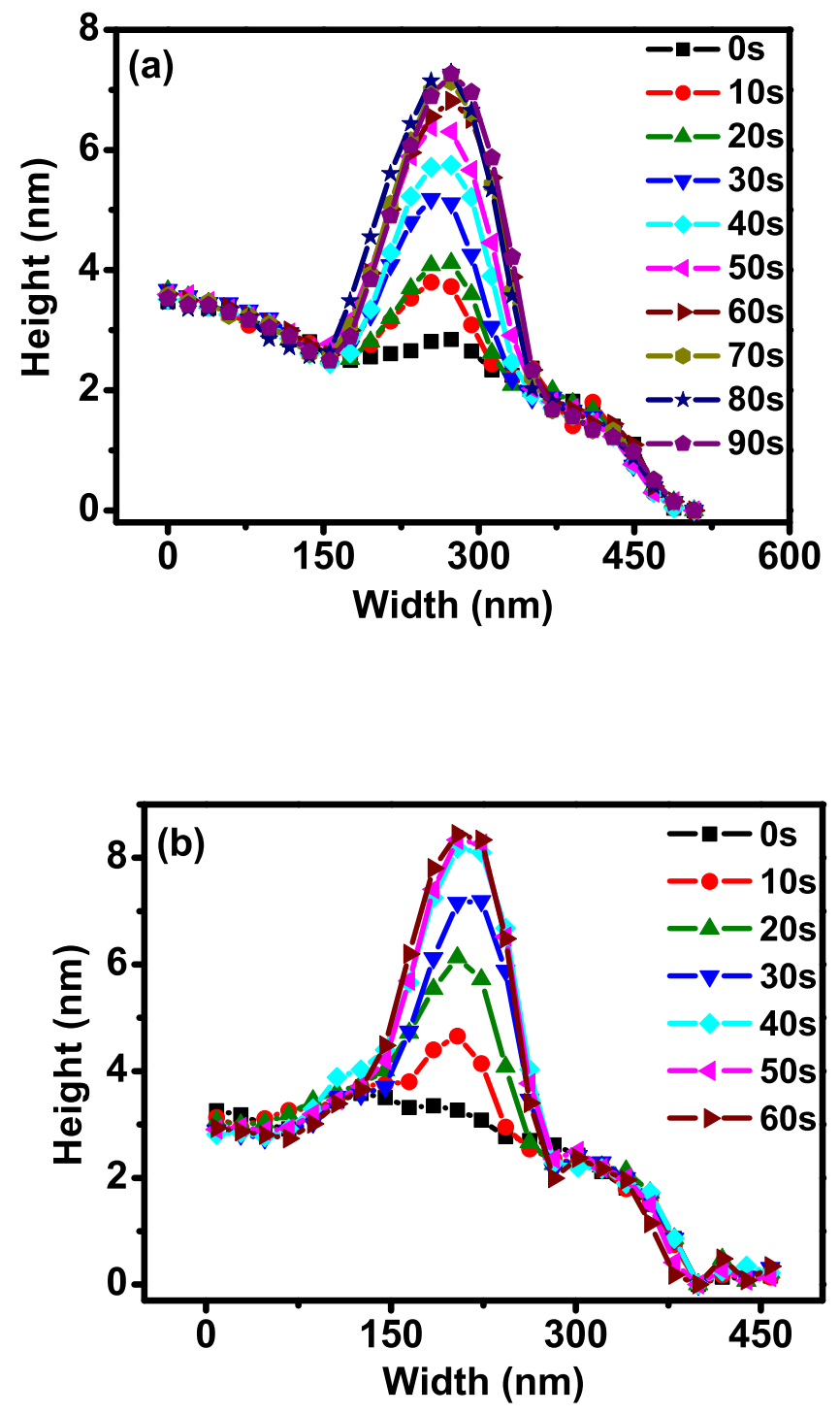

Figure 5.6: (a) shows the real-time profiles of a nanobubble on HOPG surface (as cathode) at $1 \mathrm{~V}$, with time interval of $10 \mathrm{sec}$. Another example at $2 \mathrm{~V}$ is shown in (b). By means of electrolysis of water, nanobubbles form on the surface and subsequently grow. In (a) the growth terminates after $70 \mathrm{sec}$ while in (b) this occurs already after $40 \mathrm{sec}$. The nanobubbles then remain stable. The plots also reveal that the nanobubbles grow with a higher rate in height rather than in width. 
ure 5.7(a) and (b), respectively. Exponential fits (red lines) are applied to both plots and values of the time constant $\tau$ are extracted. $\tau$ values of the area and volume growth rate are plotted versus voltage in Figure 5.7(c). The plot shows that nanobubble area and volume growth rate have a good correlation at all four voltages. This result suggests that the electrolytically generated gas is produced on the whole surface of the nanobubbles, implying that the whole surface of the nanobubbles is electrically charged. Alternatively, the electrolytically generated gas could be produced on the electrode surface (HOPG) and subsequently diffuse through the surface of the nanobubbles.

\subsubsection{Correlation between global current and local nanobubble growth}

The global current of the electrolysis system is recorded as a function of time with a sampling rate $0.367 \mathrm{sec}$ and an integration time $0.102 \mathrm{sec}$. To test the reproducibility, two HOPG samples and three freshly cleaved surfaces on each sample are analyzed (as cathode). Thus current measurements are done on six different HOPG surfaces at each voltage. All these results show that the current vs. time curves present an exponential decay at voltages below $3 \mathrm{~V}$. At higher voltages, the current fluctuates strongly. The reason is that more and bigger bubbles are formed at higher voltages. Growth and detachment of the bubbles cause the current to fluctuate. This is in the agreement with the observations in Figure 5.3 and refs. $[24,25]$.

As described in the previous section, we extract the width and height values of nanobubbles at different times, based on the AFM-recorded profiles of the nanobubbles. We here estimate the nanobubble area and aspect ratio (width over height), which are then plotted as a function of time. In Figure 5.8, graphs show the dynamics of current, nanobubble area, and nanobubble aspect ratio within the first $60 \mathrm{sec}$ at (a) $1 \mathrm{~V}$, (b) $1.5 \mathrm{~V}$, (c) $2 \mathrm{~V}$, and (d) $2.5 \mathrm{~V}$. These three quantities are recorded simultaneously at each voltage. The nanobubble development and the current decay are strongly correlated. In Figure 5.8(a), as an example, the nanobubble expands rapidly in the first $20 \mathrm{sec}$, from 20 to $50 \mathrm{sec}$ it grows less quickly, thereafter it reaches a stable state, as revealed by the area vs. time plot (red square); the current decay behaves in a correlated way on the same timescale (black dot). Interestingly, along with the current decay, 

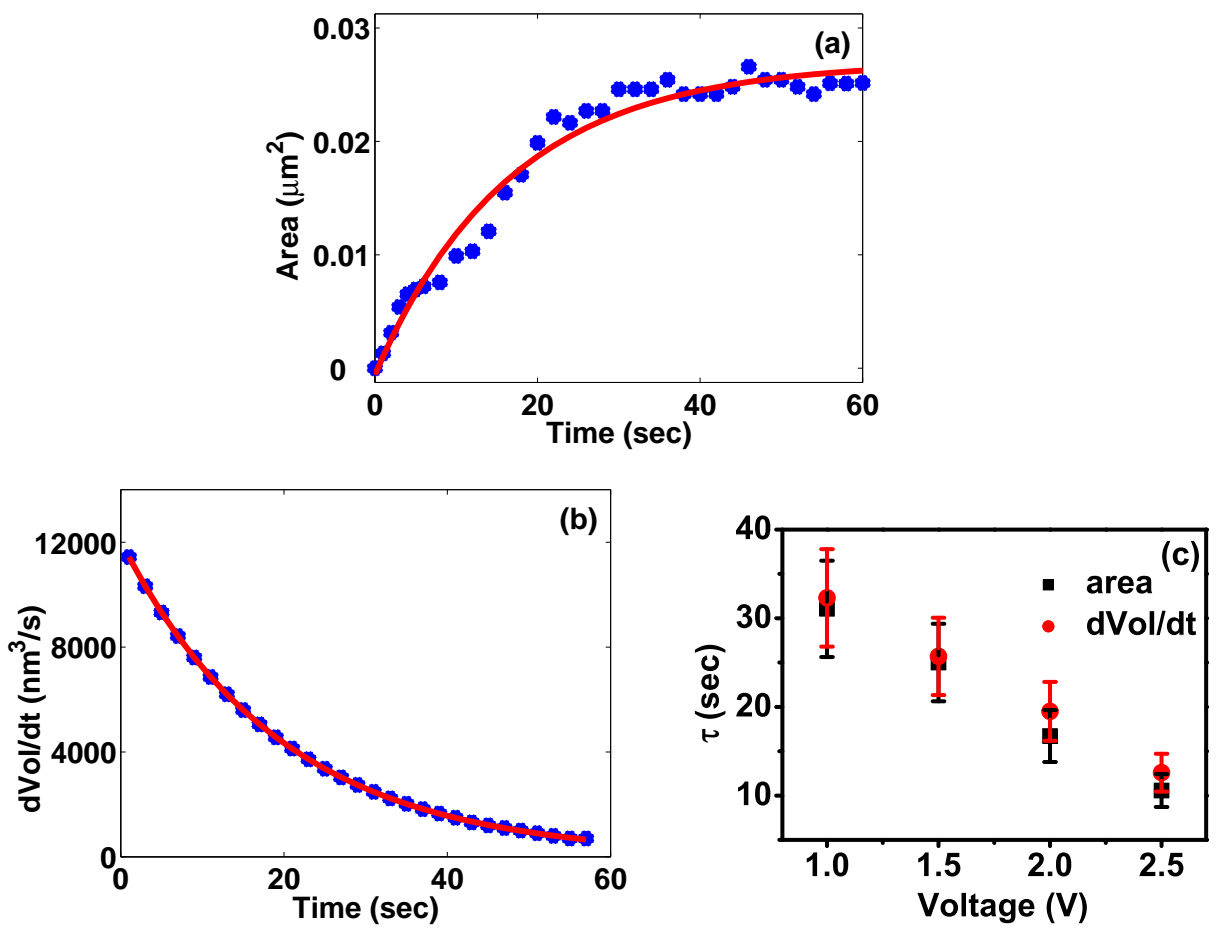

Figure 5.7: nanobubble area (a) and volume growth rate (b) plots (blue dots). The red curves are fits of an exponential function $X=X_{\infty}+\left(X_{0}-\right.$ $\left.X_{\infty}\right) e^{-t / \tau}$, where $X$ is either the area or the volume growth rate. These fits allow to define a characteristic timescale $\tau$. Values of the time constant $\tau$ extracted from the fits are exhibited as a function of the applied voltage, as shown in (c). The timescales of the area evolution (black square) and the volume growth rate evolution (red dot) show a good correlation at all voltages. This observation suggests two possible ways how the electrolytic gas is produced on the surface: i) the gas emerges at the whole surface of the nanobubbles and correspondingly the whole surface of the nanobubbles should be charged by electrons; ii) the gas emerges at the electrode surface and then diffuses through the nanobubble surface. 

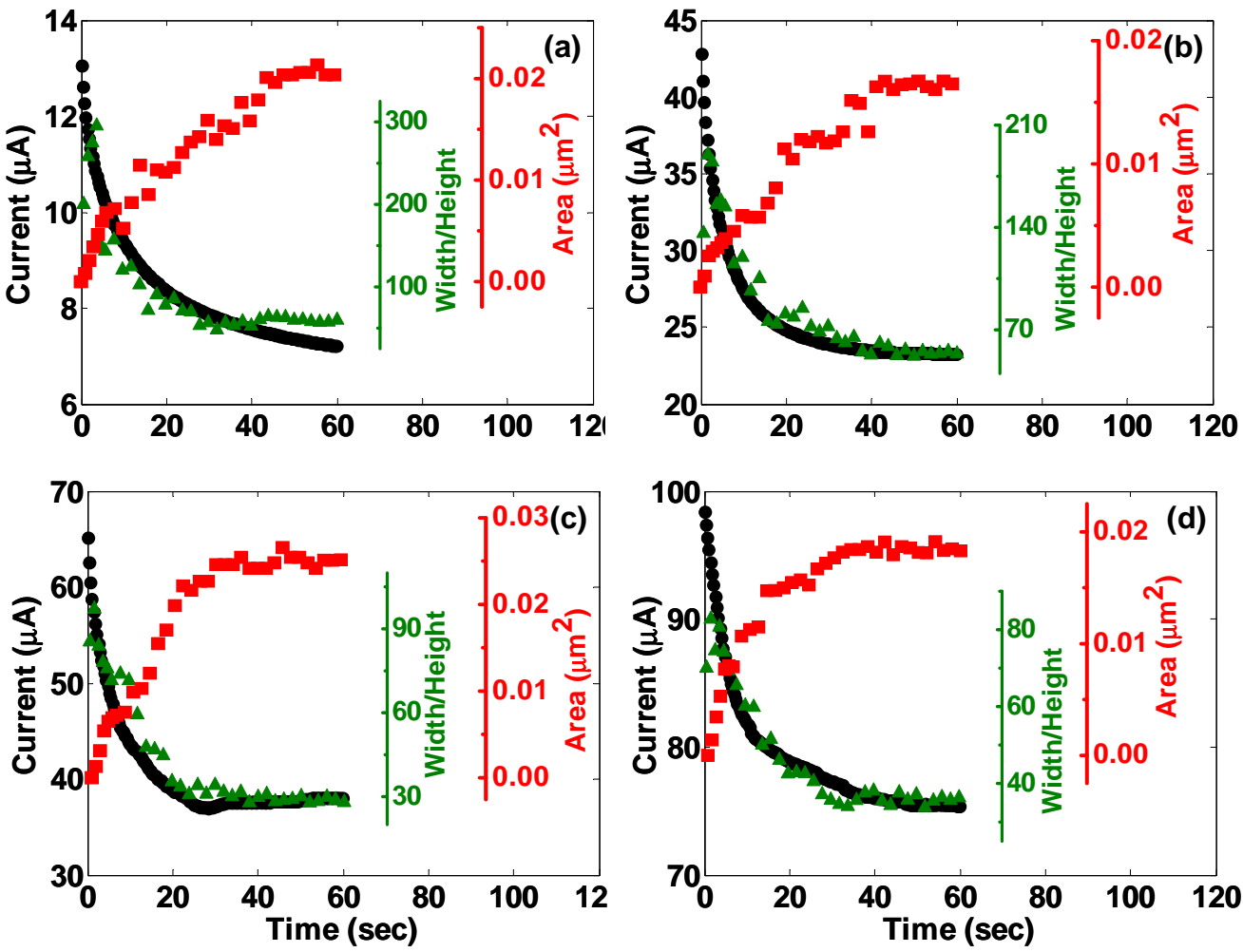

Figure 5.8: Graphs showing current, nanobubble area, and nanobubble width/height as a function of time at (a) $1 \mathrm{~V}$, (b) $1.5 \mathrm{~V}$, (c) $2 \mathrm{~V}$, and (d) 2.5 $\mathrm{V}$ on HOPG as cathode. At each voltage, the three plots are recorded simultaneously. The nanobubble development and the current decay show a clear correlation. Interestingly, the current and the nanobubble aspect ratio (green triangle) decrease in the same manner. The aspect ratio plot indicates that nanobubbles initially form in an ultrathin-film form and then accumulate with a higher rate in vertical direction rather than in horizontal; this is consistent with the findings shown in Figure 5.6. 
the nanobubble aspect ratio (green triangle) decreases too. This indicates that nanobubbles occur initially in an ultrathin-film form with a large aspect ratio, and then accumulate with a higher growth rate in vertical as compared to horizontal direction. This is consistent with the observation in Figure 5.6.

To directly show the relation between the current and the nanobubble growth, we plot the current as a function of nanobubble area or aspect ratio, as revealed in Figure 5.9. Plot (a) and (b) show how the current behaves corresponding to the development of nanobubbles when the electric potential is $1 \mathrm{~V}$. Similarly, (c) and (d), (e) and (f), and (g) and (h) are showing the plots for $1.5,2$ and $2.5 \mathrm{~V}$, respectively. The plots clearly present the fashion that the current decreases while the nanobubble area is increasing and the aspect ratio of nanobubbles is however decreasing, at all the voltages. The strong clusters appeared at the end of the plots in (b), (d), (f), and (h) indicate that the both decrease of current and the decrease of nanobubble aspect ratio reach a saturation on a similar timescale at all four voltages. This behavior also occurs in plot (e) and (g) but not in plot (a) and (c). It tells that the current saturates on a similar timescale as the developing nanobubble surface area get saturated at 2 and $2.5 \mathrm{~V}$. We will quantify this observation by applying exponential fits to the data and then extracting the time constant of the fits, as to be shown in Figure 5.11.

The gas produced at the electrode surface depends on the electric charge passing from one electrode to the other. Figure 5.8 shows that the global current reaches an equilibrium state as soon as the nanobubble development terminates. The amount of the excess electric charge above the equilibrium state within time $(60 \mathrm{sec})$ is estimated for voltage $1,1.5,2$, and $2.5 \mathrm{~V}$, respectively. The amount is plotted against the nanobubble coverage and volume at each voltage, as shown in Figure 5.10(a) and (b), respectively. The red lines are linear fits. Note that the fits are a guide to eyes, not necessarily suggesting that both coverage and volume of the nanobubbles have a linear relation with the charge. One can see that the amount of nanobubbles produced increases as the amount of excess electric charge increases, showing the contribution of the gas yielded by electrolysis to the nanobubble formation. Note the offset of the fits: in spite of the nonzero charge there is no nanobubble production (zero nanobubble coverage and volume). The offset indicates that part of the electrolytically generated gas dissolves, not contributing to the formation of nanobubbles. This crucial charge may also be needed to build up a dielectric layer at the electrode. Zhang et al. reported similar observation that 

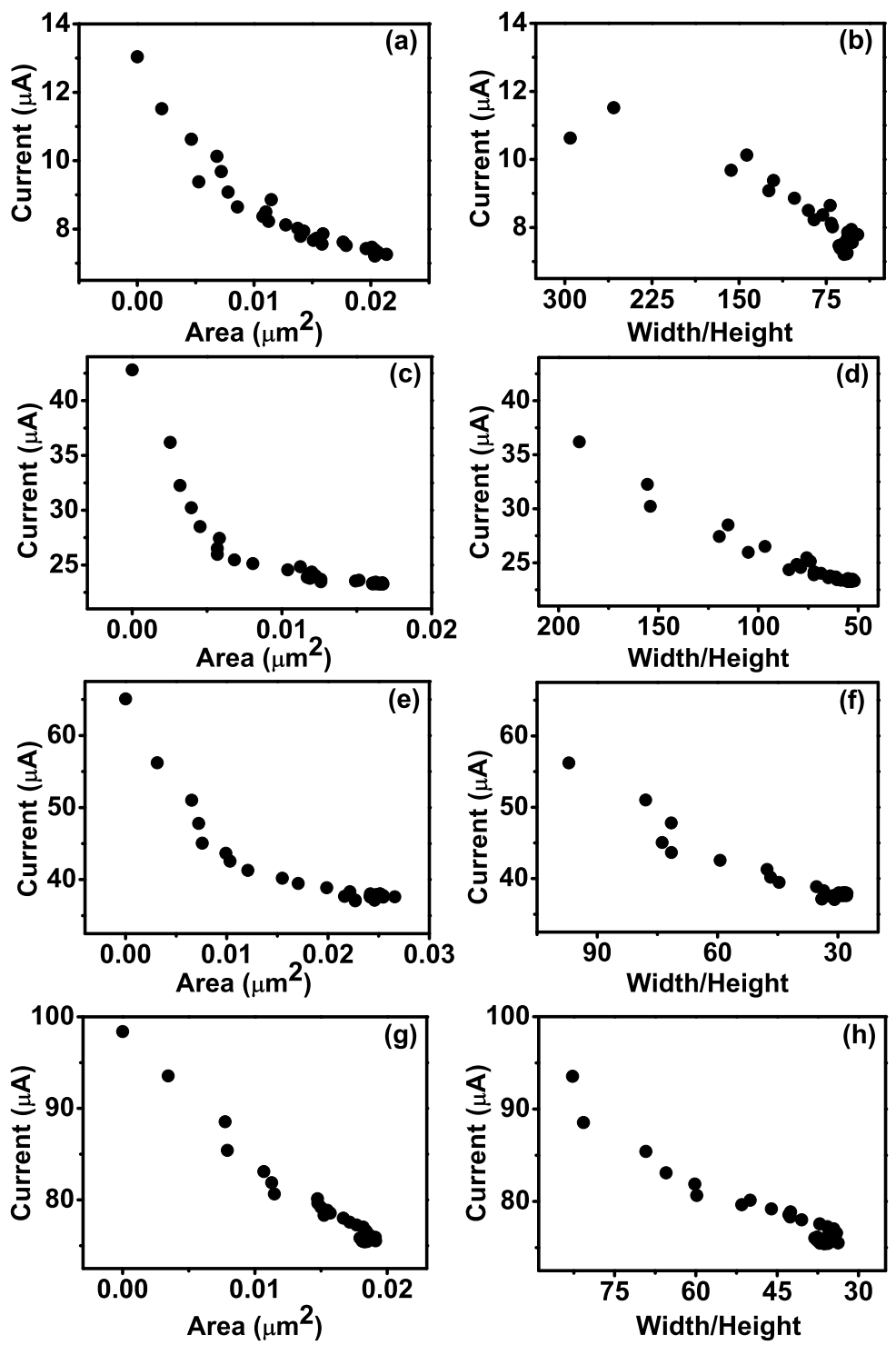

Figure 5.9: Plots showing the current as a function of nanobubble area or aspect ratio at different electric potentials: (a) and (b) for $1 \mathrm{~V}$, (c) and (d) for $1.5 \mathrm{~V}$, (e) and (f) for $2 \mathrm{~V}$, (g) and (h) for $2.5 \mathrm{~V}$. The plots reveal that the current decreases as the area increases, meanwhile the aspect ratio of nanobubbles also decreases, for all the voltages. The strong clusters at the end of the plots in (e) and (g) indicate that the decrease in current and the increase in area saturate on a similar timescale for 2 and $2.5 \mathrm{~V}$; this behavior does not occur for (a) and (c), the lower voltages. In (b), (d), (f), and (h) clusters also appear at the end of the plots, indicating that the aspect ratio and the current get saturated on a similar timescale under all four voltages. In Figure 5.11 we will show how to quantify this behavior by applying exponential fits to the data. 

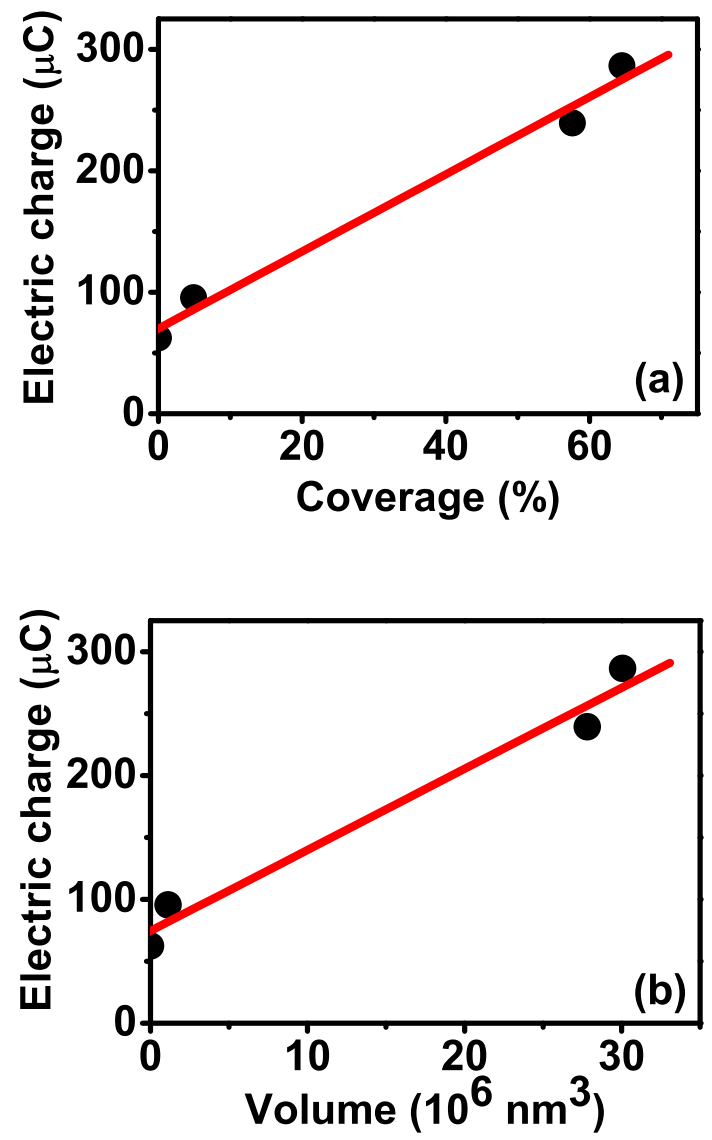

Figure 5.10: The amount of the excess electric charge in equilibrium (after $60 \mathrm{sec}$ ) is estimated for each voltage, namely $1,1.5,2$, and $2.5 \mathrm{~V}$. It is plotted versus the nanobubble coverage (a) and the volume (b). The red lines are linear fits. Note the offset of the linear fits: A finite amount of charge is needed before nanobubbles are produced, presumably in order to build up dielectric layers at the interface. 

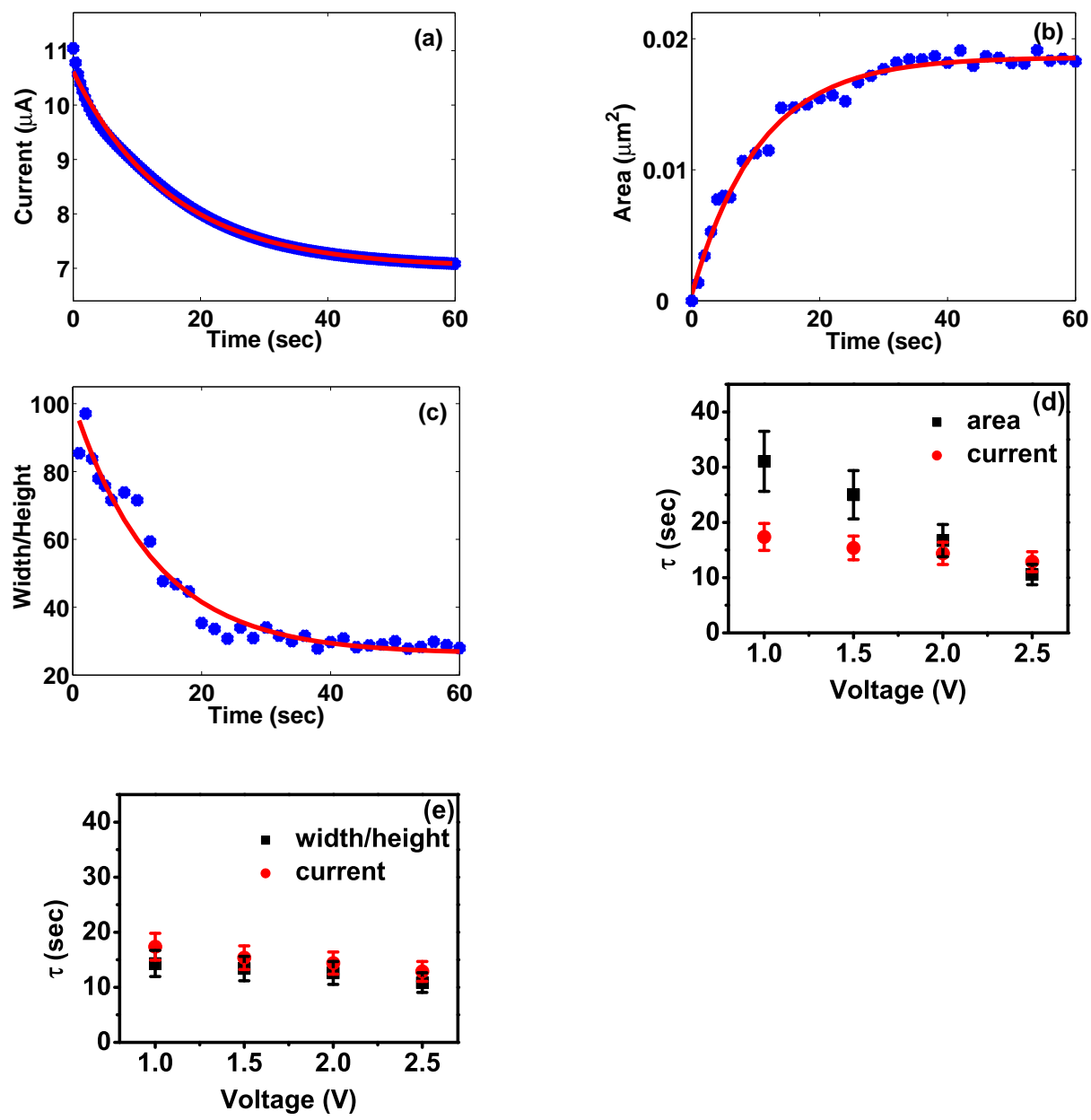

Figure 5.11: Exponential fits (red curves) of the current, nanobubble area and width/height plots (blue dots) are shown in (a), (b) and (c), respectively. Values of the time constant $\tau$ of the fits are extracted. $\tau$ is plotted versus voltage for area (error bar $\pm 17 \%$ ) and current (error bar $\pm 13 \%$ ) as shown in (d), as well as for width/height (error bar $\pm 16 \%$ ) and current as shown in (e). $\tau$ decreases with increasing voltage, this indicates that the development of nanobubbles and the decay of the current take place more rapidly at higher voltage. The $\tau$ values of the area and the current well agree at 2 and $2.5 \mathrm{~V}$ when the nanobubble coverage is high. At 1 and $1.5 \mathrm{~V}$, when nanobubble coverage is rather low, the time constants of area and current deviate. Note that the current is a global measure, whereas the area of individual nanobubbles is a local quantity. Interestingly, the nanobubble aspect ratio and the current always show good agreement (e), for which we do not have proper explanations. 
a formation time for nanobubbles is required and it decreases when the applied voltage increases [9].

For further analysis of the time scales of the current and the nanobubble growth, the current, the nanobubble area, and the aspect ratio plots are fitted with an exponential. Examples are shown in Figure 5.11(ac). Red curves are the fits to the data (blue dots), from which the time constants $\tau$ are extracted. The values of $\tau$ are presented as a function of voltage for area (error $\pm 17 \%$ ) and current (error $\pm 13 \%$ ) in Figure $5.11(\mathrm{~d})$, and for aspect ratio (error $\pm 16 \%$ ) and current in Figure 5.11(e). First, we note that the $\tau$ 's decrease with increasing voltage, indicating that the development of nanobubbles and the decay of current take place more rapidly at higher voltage. One can moreover see that the $\tau$ values of area and current: (i) agree well at 2 and $2.5 \mathrm{~V}$ when the nanobubble coverage is high; hence the nanobubble growth leads to a decrease of the current in the system; (ii) deviate at 1 and $1.5 \mathrm{~V}$, when the nanobubble coverage is rather low. We stress that the current is a global parameter, whereas the area of individual nanobubbles is a local parameter. Interestingly, the nanobubble aspect ratio and the current are perfectly correlated, as shown in Figures 5.8 and 5.11(e). We do not have an explanation for this finding. We note that the aspect ratio presumably exhibits a universal way of nanobubble development. Therefore it might be a global feature.

\subsubsection{NaCl solution as electrolyte}

To study the robustness of our observations, in addition to pure water an aqueous sodium chloride $(\mathrm{NaCl})$ solution $(0.01 \mathrm{M})$ was used as electrolyte. Using the same experimental setup as described in Figure 5.2, the $\mathrm{NaCl}$ solution is deposited on the HOPG surface acting as the negative electrode (cathode). Figure 5.12 shows the topography images of the HOPG surface at different applied electric potentials: (a) $0 \mathrm{~V}$, (b) $0.25 \mathrm{~V}$, (c) $0.5 \mathrm{~V}$, (d) $0.75 \mathrm{~V}$, (e) $1 \mathrm{~V}$, (f) $1.25 \mathrm{~V}$; the height range for each image is (a-c) $7.9 \mathrm{~nm}$ and (d-f) $10.3 \mathrm{~nm}$. The images are recorded continuously from (a) to (f), with a scanning time of $8.5 \mathrm{~min}$ per image.

The AFM images show that no nanobubbles are formed on the HOPG surface with no applied voltage. When the voltage is imposed, the formation of hydrogen nanobubbles starts to become observable. A small amount of nanobubbles are already formed at $0.25 \mathrm{~V}$. The nanobubble formation increases tremendously as the voltage is switched from 0.5 to 

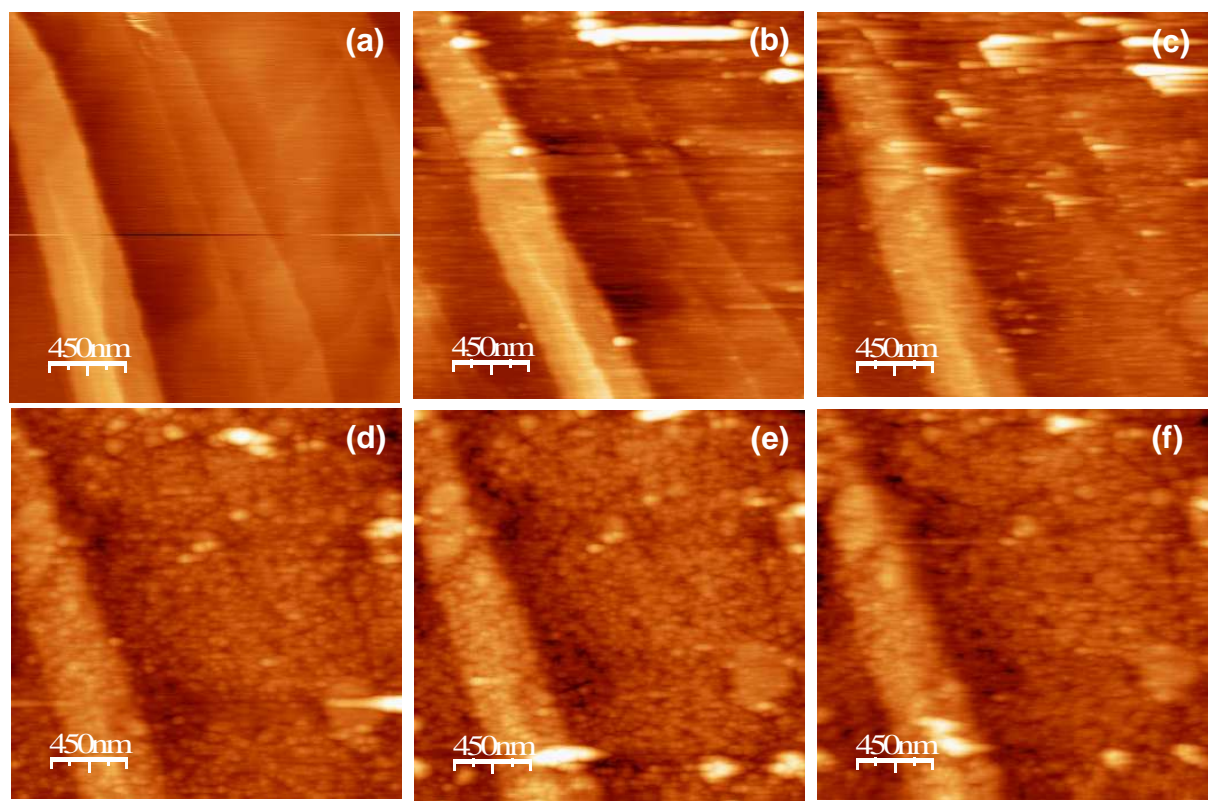

Figure 5.12: Tapping mode AFM topography images of HOPG surface under the sodium chloride $(\mathrm{NaCl})$ solution $(0.01 \mathrm{M})$. Electrolysis process is applied as the HOPG acts as cathode under different voltages: (a) $0 \mathrm{~V}$, (b) $0.25 \mathrm{~V}$, (c) $0.5 \mathrm{~V}$, (d) $0.75 \mathrm{~V}$, (e) $1 \mathrm{~V}$, (f) $1.25 \mathrm{~V}$; topographic height range of the images: (a-c) $7.9 \mathrm{~nm}$ and (d-f) $10.3 \mathrm{~nm}$. The scanning time per image is $8.5 \mathrm{~min}$ and the images are taken in a sequence from (a) to (f) with different electric potentials applied. Hydrogen nanobubbles are generated on the surface. The density of nanobubbles is enhanced with increasing voltage. Compared to the results with pure water, the nanobubbles are smaller in size. The atomic steps traversing the HOPG surface are visible even when the nanobubble coverage is very high. This shows that the height of the nanobubbles is very small. The formation of nanobubbles increases tremendously from 0.5 to $0.75 \mathrm{~V}$ and saturates after $1 \mathrm{~V}$. This is a similar observation as compared to the pure water, except that the voltage is much reduced. 

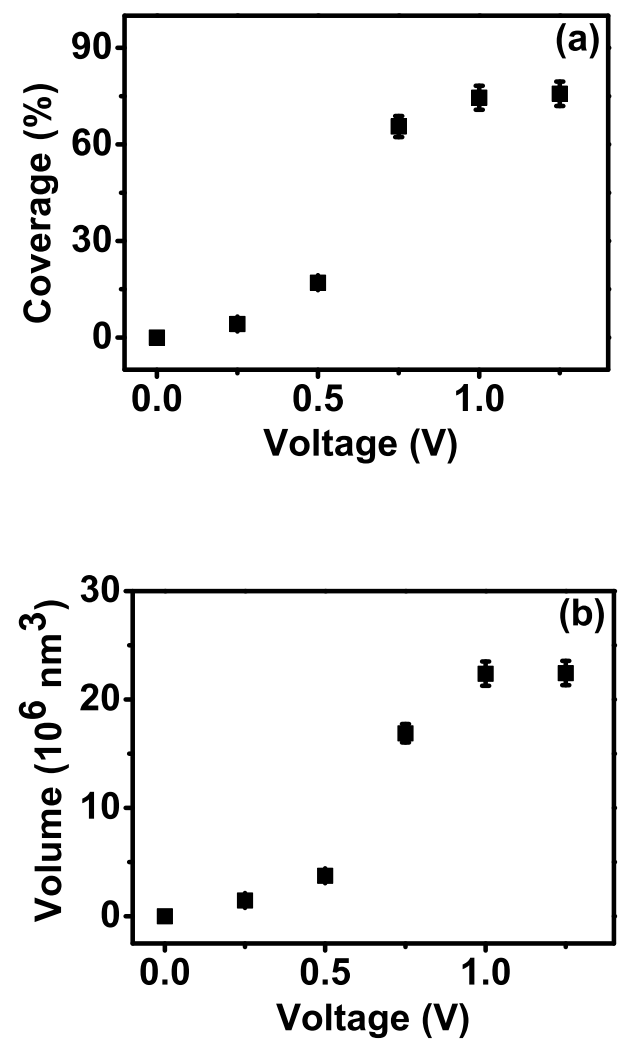

Figure 5.13: As Figure 5.12 has shown, when the $\mathrm{NaCl}$ solution solution is used instead of pure water as electrolyte, similar behavior as for pure water is observed: with increasing voltage the formation of hydrogen nanobubbles is enhanced (on HOPG as cathode). Coverage and volume of the nanobubbles are related to the applied voltage, as depicted in (a) and (b) (error bar $\pm 5 \%$ ) respectively. The required effective voltage for nanobubble creation is strongly reduced, as compared to the pure water case, as salty water has a lower resistance. 
$0.75 \mathrm{~V}$. This is similar to the result shown in Figure 5.3 where the formation of nanobubbles jumps from 1.5 to $2 \mathrm{~V}$. The formation of nanobubbles in $\mathrm{NaCl}$ solution starts to show a saturation after $1 \mathrm{~V}$. The atomic steps of HOPG are visible when the nanobubble coverage is low as well as when the nanobubbles are covering nearly the entire surface. This indicates that the height of the nanobubbles is in the comparable range of the height of atomic steps which is typically $1-3 \mathrm{~nm}$. Comparing to the nanobubbles formed with pure water, the nanobubbles in the $\mathrm{NaCl}$ solution are smaller in size. The dependence of nanobubble coverage and volume upon the applied voltage is depicted in Figure 5.13(a) and 5.13(b) (error bar $\pm 5 \%$ ), respectively. When the voltage is higher than $1.25 \mathrm{~V}$, AFM imaging is disturbed by bigger bubbles developing or detaching from the surface. The formation of nanobubbles in the $\mathrm{NaCl}$ solution is similar to that in pure water, except that because of the reduced resistance due to the dissolved salt, the effective voltage is reduced by a factor of about 3: $2 \mathrm{~V}$ for the pure water and $0.75 \mathrm{~V}$ for the $\mathrm{NaCl}$ solution. Note that the volume and coverage of nanobubbles at the effective voltages in the two cases are comparable.

The time evolution of nanobubbles at $0.25 \mathrm{~V}$ in the $\mathrm{NaCl}$ solution is shown in Figure 5.14. The nanobubbles continuously develop on the surface till $40 \mathrm{sec}$ and then remain stable, as revealed in Figure 5.14(a). As in the experiment shown in Figure 5.8, the global current of the electrolysis system, the nanobubble surface area, and the aspect ratio are measured simultaneously as a function of time within the first $60 \mathrm{sec}$, as shown in Figure 5.14(b). A good correlation between the current decay and the nanobubble development is found - this is the same observation as with pure water. The aspect ratio also shows a comparable correlation with the current. The experiments with the $\mathrm{NaCl}$ solution reproduce our findings concerning the nanobubbles in dynamic equilibrium. Again, good correlations between global current decay and bubble growth dynamics are found.

\subsection{Conclusions}

We have shown that the electrolysis of water is a reliable method to produce both hydrogen (at cathode) and oxygen (at anode) surface nanobubbles. Coverage and volume of the nanobubbles grow substantially with increasing voltage. The yield of hydrogen nanobubbles is much higher than that of oxygen nanobubbles. Our results of nanobubble evolution 

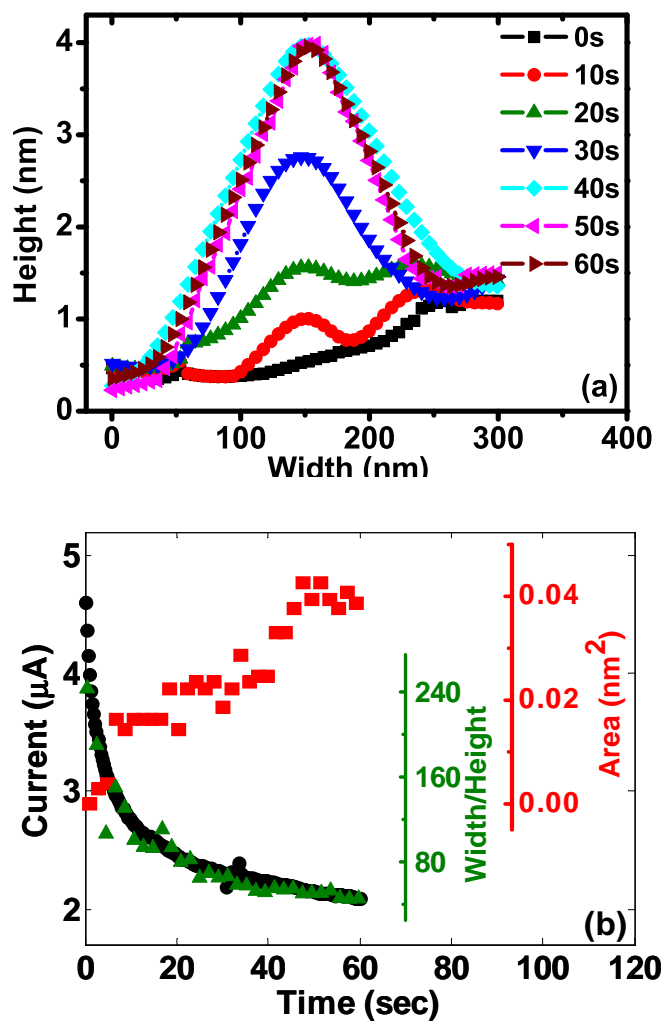

Figure 5.14: In the $\mathrm{NaCl}$ solution at $0.25 \mathrm{~V}$, as analogous to Figure 5.6 and 5.8, (a) the time evolution of a hydrogen nanobubble is recorded; (b) the current, the nanobubble area, and the width/height as a function of time are measured. The similar behaviors as for the pure water case are observed. 
have shown that nanobubbles occur initially in an ultrathin-film with a large aspect ratio, and subsequently grow with a higher rate in vertical rather than in horizontal direction. In spite of the continuously applied voltage and a nonzero current, the growth of the nanobubbles terminates after a typical time, showing that nanobubbles are in a dynamic equilibrium condition. In addition, we have found a correlation between the surface area and the volume growth rate of nanobubbles, suggesting possible ways how electrolytic gas emerges on the surface. The global current as function of time is strongly correlated with the bubble aspect ratio. The experiments with an aqueous sodium chloride solution $(0.01 \mathrm{M})$ give similar results. 


\section{References}

[1] Ball, P. Nature 2003, 423, 25.

[2] Vinogradova, O.; Bunkin, N. F; Churaev, N. V.; Kiseleva, O. A.; Lobeyev, A. V. J. Colloid Interface Sci. 1995, 173, 443.

[3] Attard, P. Adv. Colloid Interface Sci. 2003, 104, 75.

[4] Tyrrell, J. W. G.; Attard, P. Phys. Rev. Lett. 2001, 87, 176104.

[5] Ishida, N.; Inoue, T.; Miyahara, M.; Higoashitani, K. Langmuir 2000, $16,6377$.

[6] Simonsen, A.C.; Hansen, P.L.; Klösgen, B. J. Colloid Interface Sci. 2004, 273, 291.

[7] Holmberg, M.; Kühle, A.; Garnæs, J.; Mørch, K. A.; Boisen, A. Langmuir 2003, 19, 10510.

[8] Agrawal, A.; Park, J.; Ryu, D. Y.; Hammond, P. T.; Russel, T. P.; McKinley, G. H. Nano Lett. 2005, 5, 1751.

[9] Zhang, L.; Zhang, Y.; Zhang, X.; Li, Z.; Shen, G.; Ye, M.; Fan, C.; Fang, H.; Hu, J. Langmuir 2006, 22, 8109.

[10] Zhang, X. H.; Zhang, X. D.; Lou, S. T.; Zhang, Z. X.; Sun, J. L.; Hu, J. Langmuir 2004, 20, 3813.

[11] Zhang, X. H.; Maeda, N.; Craig, V. S. J. Langmuir 2006, 22, 5025.

[12] Agrawal, A.; McKinley, G. H. Mater. Res. Soc. Symp. Proc. 2006, 899E.

[13] Yang, S.; Dammer, S. M.; Bremond, N.; Zandvliet, H. J. W.; Kooij, E. S.; Lohse, D. Langmuir 2007, 23, 7072.

[14] Borkent, B. M.; Dammer, S. M.; Schönherr, H.;Vancso G. J.; Lohse, D. Phys. Rev. Lett. 2007, 98, 204502.

[15] Yang, S.; Kooij, E. S.; Poelsema, B.; Lohse, D.; Zandvliet, H. J. W. Europhys. Lett. 2008, 81, 64006.

[16] Switkes, M.; Ruberti, J. W. Appl. Phys. Lett. 2004, 84, 4759. 
[17] Steitz, R.; Gutberlet, T.; Hauss, T.; Klösgen, B.; Krastev, R.; Schemmel, S.; Simonsen, A. C.; Findenegg, G. H. Langmuir 2003, 19, 2409.

[18] Zhang, X. H.; Quinn, A.; Ducker, W. A. Langmuir 2008, 24, 4756.

[19] Borkent, B. M.; Schönherr, H.; Lohse, D. Preprint, 2008.

[20] Tsai, W. L.; Hsu, P. C.; Hwu, Y.; Chen, C. H.; Chang, L. W.; Je, J. H.; Lin, H. M.; Groso, A.; Margaritondo, G. Nature 2002, 417, 139.

[21] Boissonneau, P.; Byrne, P. J. Appl. Electrochem. 2000, 30, 767.

[22] Fan, Y.; Wang, R. Adv. Mater. 2005, 17, 2384.

[23] Volanschi, A.; Olthuis, W.; Bergveld, P. Sens. Actuators A 1996, 52, 18.

[24] Gabrielli, C.; Huet, F; Keddam, M.; Macias, A.; Sahar, A. J. Appl. Electrochem. 1989, 19, 617.

[25] Gabrielli, C.; Huet, F.; Keddam, M.; Sahar, A. J. Appl. Electrochem. 1989, 19, 683. 


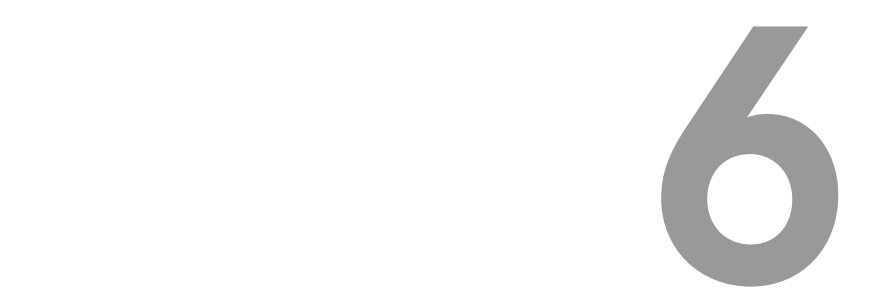

\section{Conclusion and Outlook}

\subsection{General Conclusions}

We have designed and performed experimental studies to uncover the big mysteries of the tiny bubbles, surface nanobubbles. The observations and related analyses effectively guide us to the understanding of the formation of nanobubbles. We propose the electrolysis of water as a method that can be applied to reliably control the appearance of nanobubbles for technological applications in the future.

Following our results addressed in Chapter 3, the characterization of surface nanobubbles on hydrophobilized silicon surfaces under varying conditions is achieved. We present the gas phase inside both nanobubbles and an adjacent microcavity with one single image by AFM phase measurement. A direct comparison between nanobubble and microbubble is formed, revealing the gas-liquid interface nature of the nanobubble surface. Creating nanobubbles in situ by means of heating up the substrate allows to study three aspects: i) the tendency of nanobubbles to form in the vicinity of nanogrooves on the surface; ii) a direct comparison of nanobubble profiles and the underlying substrate topography; iii) The growth and merging event of nanobubbles. The stability of nanobubbles 
is examined. The result shows that nanobubbles are very stable with a lifetime of at least a number of hours. We observe the movement of a nanobubble along a nanometer-deep groove on the substrate, demonstrating the sensitivity of nanobubbles to the surface morphology.

In addition, we find that the initial conditions during immersion are decisive for the formation of nanobubbles. The nanobubbles which are generated due to the increased water temperature (a bubble-favorable condition) do not disappear from the surface even when the water temperature drops back to ambient situation (a less favorable condition). Pressurizing the water with $\mathrm{CO}_{2}$ gas provides controlled various gas concentrations in water. We see a strong dependence of the nanobubbles on the gas concentration, in terms of nanobubble size and density. The alcoholwater-exchange method is carried out to sufficiently form surface nanobubbles; either ethanol or 2-propanol is used as the alcohol agent. Instead of pure water sodium chloride solution $(0.03 \mathrm{M})$ is added onto the surface, in which nanobubbles are formed with the same density and size as compared to the ones in pure water. Furthermore, we show that nanobubbles are sensitive to the cleaning procedure applied to prepare the substrates, i.e., the alcohol prewashing process. Density and shape of the nanobubbles are differed due to the different type of alcohols used. In terms of the effect of surfactant, addition of butanol drops to the water leads to a decrease of nanobubbles in size.

After the studies with the hydrophobic silanated wafer surfaces, we adopt highly oriented pyrolytic graphite (HOPG) sample which contains a unique surface nature, i.e., the hydrophobicity varies in a well-defined fashion due to the atomic steps traversing the surface. In Chapter 4 , we present the AFM studies of nanobubbles on the HOPG surfaces. By means of the ethanol-water-exchange process, nanobubbles are generated with a large density on the surface. As a comparison, when simply adding pure water, methanol, ethanol, 2-propanol, butanol, or performing the waterethanol-exchange process, no nanobubbles are formed on HOPG surface. The profile of nanobubbles, as imaged by AFM, is found to be sensitive to the applied interaction strength between the AFM tip and the surface. The meniscus in a manufactured microcavity presents the same property, again showing the gas-contain of nanobubbles.

The main finding in Chapter 4 is that the formation of nanobubbles shows a great enhancement at the upper side of the steps, which consists of the lowest coordinated atoms of the surface disfavoring water molecules 
- the most hydrophobic area. In contrast, no nanobubbles are generated on the lower side of the steps, which contains the highest coordinated atoms favoring water molecules - the most hydrophilic area. In addition, it is provided that the mean length of this most hydrophilic area (nanobubble-free zone) is approximately $20 \mathrm{~nm}$ from the steps. To our knowledge this work is the first correlation study between nanoscale sized topography and surface nanobubble density, and it only becomes possible by restricting us to the well-defined HOPG surfaces.

As the last part of this Thesis, in Chapter 5 we show that the electrolysis of water is a reliable method to produce both hydrogen nanobubbles at negative electrode and oxygen nanobubbles at positive electrode. However, the yield of hydrogen nanobubbles is much higher than that of oxygen nanobubbles. This is due to hydrogen's higher production in electrolysis and lower solubility in water, as comparing with oxygen. Coverage and volume of these two types of nanobubbles both show a strong dependence on the applied electric potential, i.e., the nanobubble growth is substantially enhanced with increasing voltage. By applying hold-slow function of AFM during electrolysis process, the evolution of nanobubbles with time is captured. The results show that nanobubbles occur initially in an ultrathin-film shape with a large aspect ratio, and subsequently accumulate with a higher rate in vertical rather than in horizontal direction. Moreover, in spite of the continuously applied charge flux, the development of nanobubbles terminates after a typical time period. This suggests that nanobubbles are in a dynamic equilibrium condition.

Furthermore, we find a correlation between the surface area and the volume growth rate of nanobubbles. Thus, two possible manners how electrolytic gas emerges on the surface are suggested: i) the gas is generated directly on the surface of nanobubbles; ii) the gas is generated on the electrode surface and then diffuses to the nanobubble surface. The global current through the electrolysis system is recorded during the electrolysis process, while nanobubbles are growing on the electrode surfaces. The current shows a saturation on the similar timescale of the saturation of nanobubble growth. The two simultaneously recorded quantities (the current and the nanobubble growth) reveal a correlation between the current decay and the nanobubble expansion. By extracting the time constants of the exponential fits to the data, we quantitatively verify this correlation. The nanobubble aspect ratio and the current have a good correlation under all conditions. As a contrast, the nanobubble surface area has a good correlation with the current only when the naobubble coverage is 
high enough ( $>50 \%)$. The experiments performed with the replacement of water by an aqueous sodium chloride solution $(0.01 \mathrm{M})$ reproduce the similar results.

\subsection{Outlook}

Electrolysis of water is presented to be the reliable method to produce surface nanobubbles on the electrode surfaces. The density of nanobubbles shows a strong link with the electric potential applied. The growth of nanobubbles has a good correlation with the behavior of the electric current of electrolysis. These two phenomena reveal the principle that the electrolytic gas, which is produced on the electrode surface and associated with the electric charge flux, determines the production of nanobubbles on the surface. This aspect can result in a good control on the formation of nanobubbles. Distribution, density and individual size of nanobubbles all become manipulatable. The experimental setup used in this Thesis as shown in Figure 5.2 does not offer an electric field that is well defined over the HOPG surface. In future work one can improve the quality of the electric field. The homogeneity of the electric field can be increased by applying two parallel plate electrodes; such an electric field provides homogeneous gas production over the entire electrode surface. The material property of electrode surface can be varied, e.g., by using gold or bare silicon wafer. In addition, making nanostructures or depositing various materials on electrode surface to differ local electric field can be proposed as a method to vary the nanobubble production on the same surface. Furthermore, different aqueous solutions need to be adopted as electrolyte to generate different gas types, rather than hydrogen and oxygen.

With the achievement of producing nanobubbles in a well controlled manner on surfaces by the means of electrolysis, the experiments carried out in Chapter 3 can be performed to the electrolytic nanobubbles. As we know, nanobubbles are sensitive to the hydrophobicity of the surface [1-3]. Coatings of different chemical and physical properties can be deposited onto the electrode surface in order to alter the hydrophobicity of the surface and to see how electrolytic nanobubbles respond to it. Creating nanobubbles in situ by heating up substrate is also an option for the electrolytic nanobubbles. We combine the electrolysis and substrateheating, two powerful bubble-generating methods, to allow more space 
to manipulate the formation of nanobubbles. Furthermore, the working electrolyte between two electrodes can be treated in several different ways, e.g., surfactant can be added in, temperature of the liquid can be enhanced, or one pressurizes the liquid to cause the electrolyte to contain different gases with certain concentrations. Then one studies how the electrolytic nanobubbles react to all the changes in electrolyte.

Having the results obtained from the experiments described in former paragraphs, one will be offered an insight to the nature of nanobubbles to answer the questions that have been always asked, i.e., what forms and stabilizes those bubbles that should not exist, what causes nanobubbles to have such a large nanoscopic contact angle $\left(\sim 170^{\circ}\right)$ as the macroscopic contact angle on the same surface is much smaller $\left(\sim 100^{\circ}\right)[2$, $4,5]$. In Chapter 5, based on our observations we suggested that nanobubbles are in a dynamic equilibrium state. Yet, some basic questions still need to be answered, for instance, what drives the gas-flows into and out of nanobubbles in the thermodynamic point of view? The answer to this question will be obtained after more studies are performed on the electrolytic nanobubbles, e.g., to explore how the development-saturated nanobubbles react to a sudden substrate temperature change.

Nanobubbles are linked to a number of phenomena associated with liquid-solid interfaces, e.g., the attractive force between hydrophobic walls in solution [6-8], and the slip condition at solid-liquid interface [9-12]. We now know that nanobubbles can be electrically produced with controlled distributions and individual shapes with the help of electrolysis. Thus, one can measure the hydrophobic force and the slip length at the desired conditions of nanobubbles, in terms of, for instance, different nanobubble density and height, or localized surface nanobubble patterns. The measurements will provide understandings in these phenomena. As a result, there will be developments of novel techniques in detecting and controlling slip velocity and hydrophobic forces on micro- and nanoscales. This helps to promote technologies of building innovative devices in nanofluidic systems such as medical detectors, automotive sensors, inkjet printing unit, and biological sensors. 


\section{References}

[1] Agrawal, A.; Park, J.; Ryu, D. Y.; Hammond, P. T.; Russel, T. P.; McKinley, G. H. Nano Lett. 2005, 5, 1751.

[2] Zhang, X. H.; Maeda, N.; Craig, V. S. J. Langmuir 2006, 22, 5025.

[3] Yang, S.; Kooij, E. S.; Poelsema, B.; Lohse, D.; Zandvliet, H. J. W. Europhys. Lett. 2008, 81, 64006.

[4] Simonsen, A.C.; Hansen, P.L.; Klösgen, B. J. Colloid Interface Sci. 2004, 273, 291.

[5] Yang, S.; Dammer, S. M.; Bremond, N.; Zandvliet, H. J. W.; Kooij, E. S.; Lohse, D. Langmuir 2007, 23, 7072.

[6] Tyrrell, J. W. G.; Attard, P. Phys. Rev. Lett. 2001, 87, 176104.

[7] Parker, J. L.; Claesson, P. M.; Attard, P. J. Phys. Chem. 1994, 98, 8468.

[8] Attard, P. Langmuir 1996, 12, 1693.

[9] Lauga, E.; Brenner, M. P.; Stone H. A.; in Handbook of Experimental Fluid Dynamics, edited by Tropea, C.; Foss, J.; Yarin A.,Springer, New York 2005.

[10] Bonaccurso, E.; Butt, H. J.; Craig, V. S. J. Phys. Rev. Lett. 2003, 90, 144501.

[11] Neto, C.; Evans, D. R.; Bonaccurso, E.; Butt, H. J.; Craig, V. S. J. Rep. Prog. Phys. 2005, 68, 2859.

[12] de Gennes, P. G. Langmuir 2002, 18, 3413. 


\section{Summary}

This Thesis focused on surface nanobubbles that posted puzzling fundamental questions and potentially would have wide technical applications. Chapter 1 was the introductory part. It summarized the previous findings reported in literature and demonstrated the quickly expanding interests in surface nanobubbles. We addressed the nanobubble-generated questions which were then followed by the content guidance explaining the work done in the Thesis and the related motivations.

AFM in tapping mode allowed to delineate wet surfaces on nanometer scale, with limited tolerances in chemical, temperature, and electrical diversities. The microscope was the key instrument we used to study nanobubbles. The AFM not only detected nanobubbles but also offered possibilities to manipulate nanobubbles with its cantilever. Due to the dimension of the nanobubbbles, the measurements obtained with AFM were circumstantial and indirect. Therefore, appropriate understanding and operating of AFM were primary issues in the work. In Chapter 2, we gave the introduction of AFM. The working principle, the operation modes and the advantages as well as the limitations of AFM in surface studies were addressed. We concisely discussed the current development of AFM and emphasized its operation in fluids, at the end of this Chapter.

We characterized the nanobubbles on hydrophobic surfaces in Chapter 3. The silane-coated silicon wafer with hydrophobic nature favored the formation of nanobubbles. Hence it served as the bubble-forming substrate. We mastered the preparation method of the surface, which was also addressed in this Chapter. Nanobubbles were imaged next to microcavities that trapped gas inside, in order to test the gas property of nanobubbles. The lifetime of nanobubbles on the surface was examined on timescale of hours. We found a link between surface roughness and nanobubble formation. Increasing substrate temperature formed nanobubbles in situ, in which experiment the merging and development of 
nanobubbles were observed. In addition, remarkably the surface topography exactly underneath nanoubbbles was delineated. Furthermore, we showed that the surface pre-treatments were influential on nanobubbles in density and size.

Chapter 3 also discussed the effects of liquid conditions on nanobubbles. We studied the effect of water temperature, gas concentration, surfactant added in water, and the ethanol-water-exchange process on the formation of nanobubbles. Increasing water temperature facilitated the formation of nanobubbles. Also, we found that nanobubbles did not disappear when the water cooled down to ambient conditions. We pressurized the water with $\mathrm{CO}_{2}$ at different pressures in order to achieve a variable gas concentration with which nanobubble density and size both were varied. Adding butanol drops caused a decrease of radius of nanobubble curvature. The ethanol-water-exchange process dramatically enhanced nanobubble formation.

Applying the ethanol-water-exchange process, nanobubbles were efficiently formed on HOPG surfaces. In Chapter 4, We showed that the distribution of nanobubbles was inhomogeneous on the surface of HOPG. This was caused by the atomic steps. The formation of nanobubbles was strongly enhanced at the upper side of the atomic steps, i.e., the most hydrophobic area on the surface. In contrast, no nanobubbles were formed at the lower side of the steps, i.e., the most hydrophilic area $(\sim 20 \mathrm{~nm}$ wide), and the terrace was intermediate. In addition, we showed that the profile of nanobubbles was sensitive to the applied AFM tip-force, demonstrating the deformability of nanobubbles. Similar measurements were carried out on a solid feature and a meniscus in microcavity as comparisons.

The work presented in Chapter 5 showed that electrolysis of water was a reliable method that led to control the appearance and growth of nanobubbles. We observed that both oxygen (at anode) and hydrogen (at cathode) nanobubbles were produced by electrolysis of water. Nanobubble coverage, volume, and size were varied with regard to the different electric potentials applied. In this Chapter, we presented the real-time development of nanobubbles and the decay behavior of the global electric current. We found a correlation between the nanobubble development and the current decay. With the results, we suggested possible mechanisms how gas was generated on the electrode surface and how nanobubbles remained in equilibrium. An aqueous sodium chloride solution offered the 
similar observations as compared to the pure water used as electrolyte.

All the work described in this Thesis, which consequently will bring us the benefits in developing innovative technologies for micro- and nanofluidic systems, was concluded in Chapter 6. 


\section{Samenvatting}

Dit proefschrift heeft betrekking op het onderzoek naar oppervlakte nanobellen. Afgezien van het feit dat dit onderwerp fundamenteel gezien zeer intrigerend is, zijn er vanuit technologisch oogpunt ook een aantal interessante aspecten die het doen van onderzoek naar dit onderwerp rechtvaardigt. In hoofdstuk 1 wordt een inleiding gegeven over dit onderwerp, waarbij de reeds voorhanden zijnde literatuur kort wordt besproken. Er is speciale aandacht voor de motivatie en de nog openliggende vragen binnen dit thema.

AFM (Atomic Force Microscopy) bedreven in de trillende cantilever mode is een uiterst attractieve techniek om oppervlakken in natte omgeving op nanoschaal af te beelden. Vandaar dat in dit onderzoek voor deze techniek is gekozen. We kunnen de nanobellen niet alleen afbeelden, maar we kunnen ze ook manipuleren en deformeren door slim gebruik te maken van de mogelijkheden van de AFM. Het moge duidelijk zijn dat een goede introductie van deze techniek dus niet mag ontbreken in dit proefschrift. In hoofdstuk 2 bespreken we niet alleen de huidige stand van zaken met betrekking tot de AFM techniek, maar ook de voor- en nadelen van deze techniek zullen de revue passeren.

Hoofdstuk 3 handelt over het ontstaan en het gedrag van nanobellen op hydrofobe oppervlakken. Silicium plakken, gefunctionaliseerd met alkaansilaan-moleculen, zijn hydrofoob en dus bij uitstek geschikt voor het ontstaan van nanobellen. We bespreken onder andere de preparatie van deze oppervlakken. Verder hebben we het ontstaan van nanobellen bestudeerd in de nabijheid van microholtes, welke gevuld zijn met gas. Op deze manier leren we het één en ander over de relatie tussen de aanwezigheid van gas en het ontstaan van nanobellen. Om een indruk te krijgen van de levensduur van de gevormde nanobellen hebben we de belletjes voor enkele uren gevolgd. Er bleek een verband te bestaan tussen het ontstaan van nanobellen en de ruwheid van het oppervlak. Door een 
toename van de temperatuur kon in situ de productie van nanobellen worden verhoogd. We hebben vervolgens niet alleen de groei, maar ook het s̈amensmeltenv̈an nanobellen nauwkeurig in kaart gebracht. Tevens zijn we erin geslaagd om het oppervlak onder de nanobellen te bestuderen. Tot slot bleek dat de precieze voorbehandeling van invloed was op dichtheid en de grootte van de nanobellen. In dit hoofdstuk hebben we ook de invloed van de vloeistof op de nanobellen aan een nader onderzoek onderworpen. Zo hebben we de gasconcentratie, de temperatuur van het water en de aanwezigheid van surfactants gevarieerd om een beter beeld te krijgen van de rol die de vloeistof in dit proces speelt. Een stijgende temperatuur leidde tot een hoger dichtheid aan nanobellen, terwijl het vervolgens afkoelen van de vloeistof geen effect had op de nanobellen. De concentratie $\mathrm{CO}_{2}$ in het water is gevarieerd om zo het effect op de dichtheid en grootte van de nanobellen te bestuderen. De toevoeging van enkele druppels butanol zorgde voor een afname van de kromtestraal van de nanobellen. Bovendien hebben we het ethanol-water verwisselingsproces onder de loep genomen. Het toepassen van deze truc op een HOPG (Highly Oriented Pyrolytic Graphite) oppervlak leidde tot een dramatische toename van het aantal nanobellen.

In hoofdstuk 4 tonen we aan dat de productie van nanobellen op een HOPG oppervlak niet uniform is. Op de meest hydrofobe gedeelten van het oppervlak, namelijk de bovenzijde van atomaire stappen, blijken veel meer nanobellen te ontstaan dan op de terrassen. Aan de voet van de atomaire stappen blijken in een zone van ca. $20 \mathrm{~nm}$ helemaal geen nanobellen te ontstaan. Juist deze gebieden zijn het meest hydrofiel. Verder laten we zien dat het profiel van de nanobellen afhangt van de door de AFM naald aangebrachte kracht. Vergelijkbare metingen zijn uitgevoerd op vast uitstulpingen van het oppervlak en de meniscus van een microholte.

Het werk dat in hoofdstuk 5 is beschreven toont aan dat elektrolyse een betrouwbare methode is voor de gecontroleerde productie van nanobellen. De elektrolyse van water leidt zowel tot zuurstof nanobellen (bij de anode) alsook tot waterstof nanobellen (bij de kathode). Afhankelijk van aangelegde elektrische spanning zijn de bedekking, het volume en de grootte van de nanobellen bestudeerd. We hebben een link weten te leggen tussen de tijdsafhankelijk ontwikkeling van de nanobellen en de verandering van de totale elektrische stroom. Gebruik makend van deze metingen konden we een mechanisme voorstellen voor hoe precies het gas aan het oppervlak wordt gegenereerd en hoe de nanobelletjes sta- 
biel kunnen blijven. Metingen in een waterige zoutoplossing, met hogere geleidbaarheid, in plaats van puur water gaf ons beter inzicht in het proces.

Dit werk heeft ons voordelen gebracht die helpen bij de ontwikkeling van innovatieve technologie en van micro- en nanovloeistof systemen. Deze overwegingen en een aantal andere bevindingen worden beschreven in het afsluitende hoofdstuk 6 . 


\section{Acknowledgements}

This work is part of the research program of the Stichting voor Fundamenteel Onderzoek der Materie (FOM), which is financially supported by the Nederlandse Organisatie voor Wetenschappelijk Onderzoek (NWO). It was carried out at the Physics of Fluids group (POF) and Solid State Physics group (SSP) in Faculty of Science and Technology, University of Twente. I gratefully acknowledge the support of these institutions.

As in retrospect of the past four years, I realize that this thesis presents the sum of the efforts of a large number of people. Hereto I would like to thank all the people who have been involved to make the accomplishment of this thesis appear.

First of all, I would like to express my gratitude to Prof. Detlef Lohse and Prof. Harold Zandvliet. I thank both of you for educating me the aspects in doing research work. You have always supported and encouraged me to explore the world of Physics. You also influenced me with your great personal values and I always felt honored to be your student. I hope that in the future our paths will continue to cross, in personal and professional atmospheres.

I greatly thank Dr. Stefan Kooij, Prof. Bene Poelsema, and Prof. Andrea Prosperetti for their kind guidance on me and their involvements in this work over the past four years.

My enormous thankfulness are given to my teamworkers Dr. Peichun Tsai, Bram Borkent, Dr. Nicolas Bremond, and Dr. Stephan Dammer. Only with the uncountable efforts from all you, we together have achieved the results of this work.

I also thank Dr. Chao Sun, Dr. Valeria Garbin, Dr. Mauro Sbragaglia, Sissi de Beer, and Dr. Christophe Pirat for the stimulating discussions that we had. They helped to generate innovative ideas for the experiments.

The thanks are emphasized to Joanita Leferink (POF), Rianne Nales 
(SSP), and Maria Teuwissen (FOM) for all the administrative issues they have always been able to quickly take care of; it has been fantastic and very helpful. I would also like to thank the technicians who have helped to make this thesis possible, Herman Oerbekke, Hans Bevers, Gerard Kip, and Geert Mentink at SSP; Gert-Wim Bruggert, Martin Bos, and Bas Benschop at POF.

Doing research work is even more delightful and practically handier when one has nice colleagues. I gratefully acknowledge all the members in POF group and SSP group. Special thanks are given to my officemates Bram Borkent, Maeva Deguette, Mauro Sbragaglia, Rory Dijkink, Christophe Pirat, Todd Hay, Tak-Shing Chan and Aurore Naso, as well as to my laboratory-mates Georgiana Stoian and Amir Saedi for numerous scientific and personal lively conversations. I also would like to thank my teammates in Physics of Fluids United soccer team which by the way is an uprising competitor on campus.

Finally, I deeply thank my family for the support and understanding that they have given to me during all the years of my study. 


\section{About The Author}

Shangjiong Yang was born on March 3rd, 1980 in Chongqing City, China. He is Tinzhong Yang and Wenqin Zhang's son and the younger brother of Yong Yang's. After wonderful years in kindergarten and primary school, he entered Nankai Middle and High School in Chongqing where he finished the six-year-long study in 1998. After the high school he was accepted by Zhejiang University in China. He studied materials science and engineering with the enjoyments of undergraduate student activities. In Zhejiang University he obtained his Bachelor in Engineering in 2002. In the same year, he entered Christian-Albrechts-University of Kiel in Germany for a master program. He did his master thesis with Prof. Wolfgang Jäger on the topic "characterization of nanolayers on structured silicon substrate for X-ray optics". In 2004 he received his Master in Science and moved to Physics of Fluids group in University of Twente, Netherlands, where he started the PhD project with Prof. Detlef Lohse. Under the guidance of Prof. Harold Zandvliet and Prof. Detlef Lohse, Shangjiong Yang worked on surface science and nanofluidics. In 2008 he accomplished his doctoral dissertation "manipulating surface nanobubbles". 